\title{
MILLIMETER WAVE RADIO FREQUENCY PROPAGATION MODEL DEVELOPMENT
}

Youssef Tawk and Christopher Romero

University of New Mexico

1700 Lomas Blvd NE

Albuquerque, NM 87106-3807

28 Aug 2014

Final Report

APPROVED FOR PUBLIC RELEASE; DISTRIBUTION IS UNLIMITED.

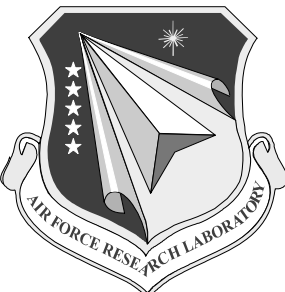

AIR FORCE RESEARCH LABORATORY Space Vehicles Directorate 3550 Aberdeen Ave SE AIR FORCE MATERIEL COMMAND KIRTLAND AIR FORCE BASE, NM 87117-5776 


\section{DTIC COPY NOTICE AND SIGNATURE PAGE}

Using Government drawings, specifications, or other data included in this document for any purpose other than Government procurement does not in any way obligate the U.S. Government. The fact that the Government formulated or supplied the drawings, specifications, or other data does not license the holder or any other person or corporation; or convey any rights or permission to manufacture, use, or sell any patented invention that may relate to them.

This report is the result of contracted fundamental research deemed exempt from public affairs security and policy review in accordance with SAF/AQR memorandum dated 10 Dec 08 and AFRL/CA policy clarification memorandum dated 16 Jan 09. This report is available to the general public, including foreign nationals. Copies may be obtained from the Defense Technical Information Center (DTIC) (http://www.dtic.mil).

\section{AFRL-RV-PS-TR-2014-0044 HAS BEEN REVIEWED AND IS APPROVED FOR PUBLICATION IN ACCORDANCE WITH ASSIGNED DISTRIBUTION STATEMENT.}

//SIGNED//

STEVEN A. LANE

Program Manager
//SIGNED//

PAUL HAUSGEN

Technical Advisor, Spacecraft Component Technology Branch

//SIGNED//

BENJAMIN M. COOK, Lt Col, USAF

Deputy Chief, Spacecraft Technology Division

Space Vehicles Directorate

This report is published in the interest of scientific and technical information exchange, and its publication does not constitute the Government's approval or disapproval of its ideas or findings. 


\section{REPORT DOCUMENTATION PAGE}

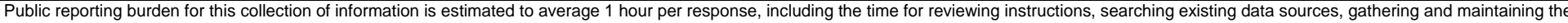

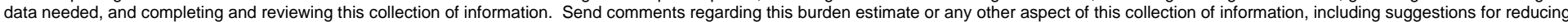

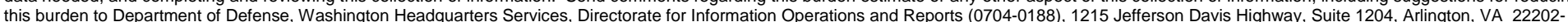

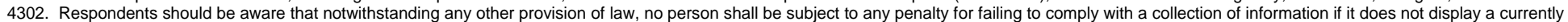
valid OMB control number. PLEASE DO NOT RETURN YOUR FORM TO THE ABOVE ADDRESS.

\begin{tabular}{l|l|l}
\hline 1. REPORT DATE $(D D-M M-Y Y Y Y)$ & 2. REPORT TYPE & 3. DATES COVERED (From - To) \\
$28-\odot 8-2014$ & Final Report & $21-$ Mar -2013 to $28-\mathrm{Feb}-2014$
\end{tabular}

4. TITLE AND SUBTITLE

MILLIMETER WAVE RADIO FREQUENCY PROPAGATION MODEL DEVELOPMENT 5a. CONTRACT NUMBER FA9453-13-1-0230 5b. GRANT NUMBER

\section{AUTHOR(S)}

Youssef Tawk and Christopher Romero

\section{5c. PROGRAM ELEMENT NUMBER $63401 \mathrm{~F}$}

5d. PROJECT NUMBER

$682 \mathrm{~J}$

5e. TASK NUMBER

PPM00015916

5f. WORK UNIT NUMBER

EF008901

\section{PERFORMING ORGANIZATION NAME(S) AND ADDRESS(ES)}

University of New Mexico

1700 Lomas Blvd NE

Albuquerque, NM 87106-3807

9. SPONSORING I MONITORING AGENCY NAME(S) AND ADDRESS(ES)

Air Force Research Laboratory

Space Vehicles Directorate

3550 Aberdeen Ave SE

Kirtland AFB, NM 87117-5776

8. PERFORMING ORGANIZATION REPORT NUMBER

\section{DISTRIBUTION / AVAILABILITY STATEMENT}

Approved for Public Release; Distribution is Unlimited.

\section{SUPPLEMENTARY NOTES}

\section{ABSTRACT}

The $V$ and $W$-bands are becoming of interest to satellite designers due to the large availability of bandwidth and the potential multi-gigabit data rates. Implementation of a satellite communication channel at these frequencies has not been documented in literature. Millimeter waves are affected a lot by atmospheric attenuation, with rain being the most detrimental and uncertain. Predictive models of rain attenuation claim some degree of accuracy up to $55 \mathrm{GHz}$, although they are primarily based on measurements at $40 \mathrm{GHz}$ and below. This project focuses on the analysis and verification of propagation models for electromagnetic waves through the atmosphere at millimeter wavelengths. Tests were performed below $40 \mathrm{GHz}$, but as a precursor for testing below $90 \mathrm{GHz}$.

\section{SUBJECT TERMS}

Space communication, satellite communication, millimeter wave, wave propagation

16. SECURITY CLASSIFICATION OF:

a. REPORT Unclassified b. ABSTRACT

Unclassified c. THIS PAGE

Unclassified

\section{LIMITATION} OF ABSTRACT

Unlimited

\section{NUMBER} OF PAGES

122
10. SPONSOR/MONITOR'S ACRONYM(S)

AFRL/RVSV

1. SPONSOR/MONITOR'S REPORT NUMBER(S)

AFRL - RV - PS - TR - 2014 - $\odot ४ 44$ 
(This page intentionally left blank) 


\section{TABLE OF CONTENTS}

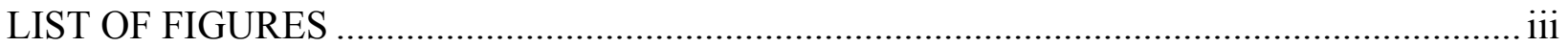

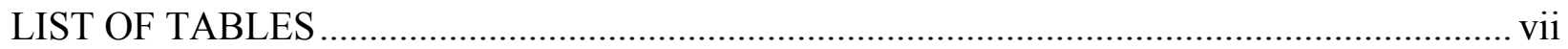

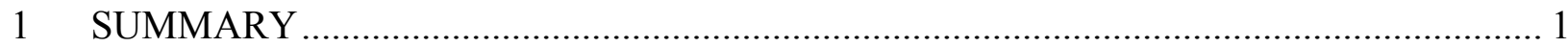

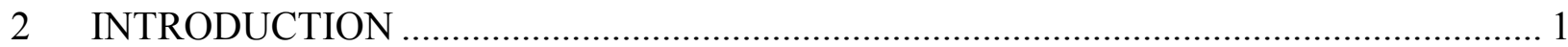

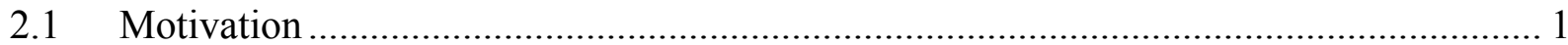

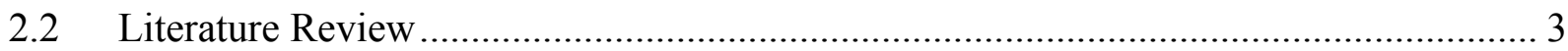

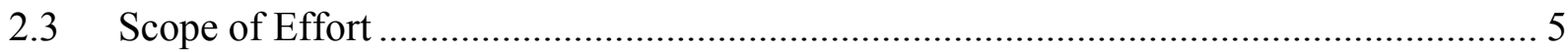

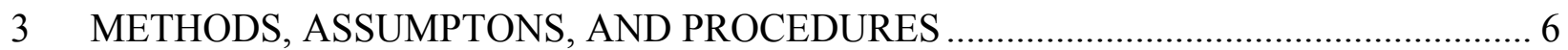

3.1 Coupled Model Development and Link Analysis ............................................................. 6

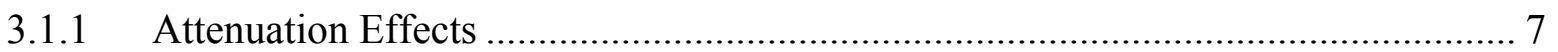

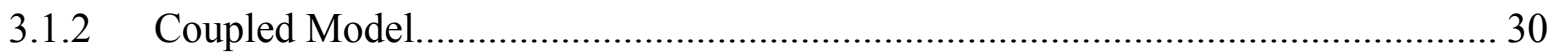

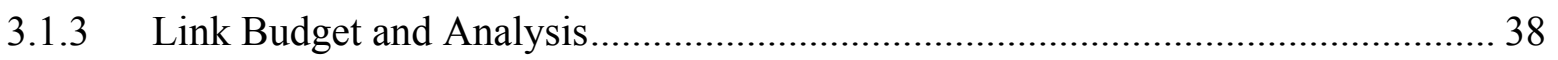

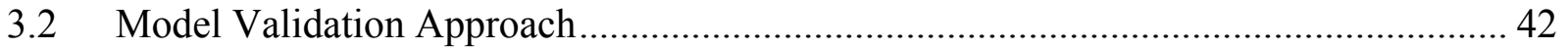

3.3 Experiment 1 - Measuring Propagation Losses............................................................. 42

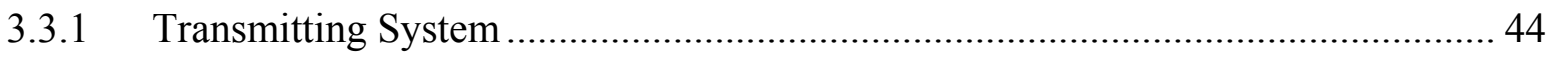

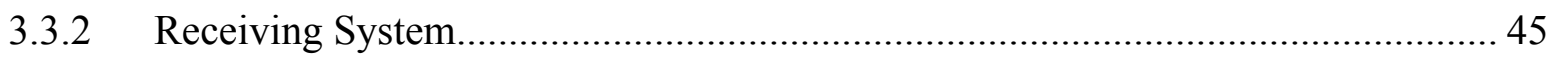

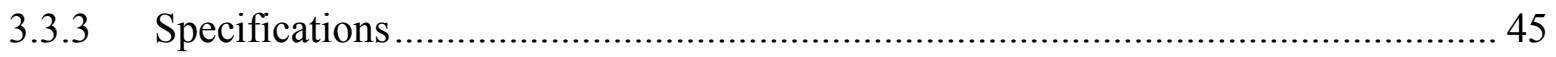

3.4 Experiment 2 - Quantifying Accuracy in an Anechoic Chamber................................... 47

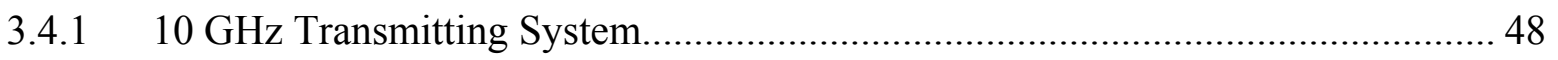

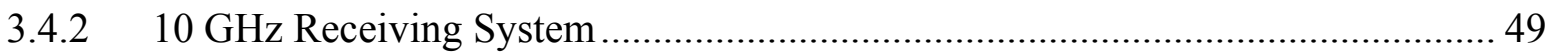

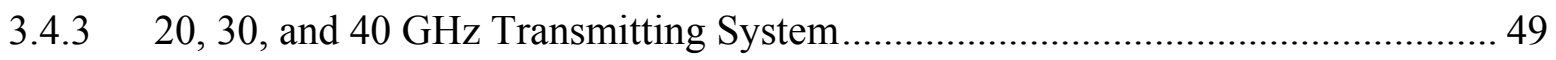

3.4.4 20, 30, \& $40 \mathrm{GHz}$ Receiving System............................................................. 49

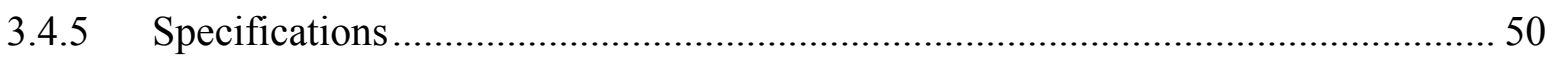

3.5 Experiment 3 - Quantifying Model Accuracy with Rain Impairment........................... 55

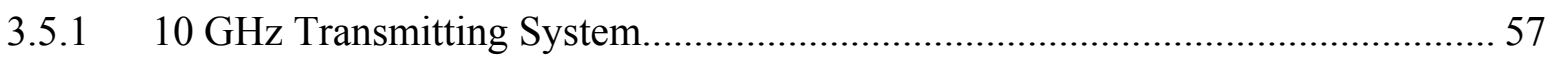

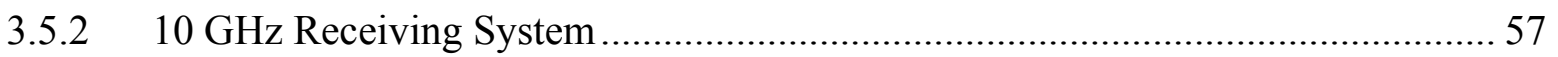

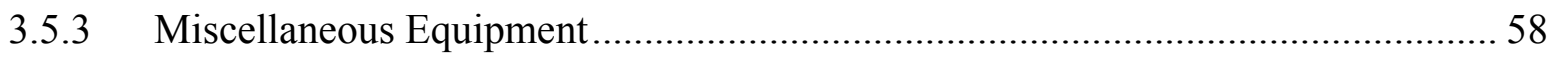

3.5.4 Input Parameters and Predicted Performance …………………......................... 58

3.6 Experiment 4 - Quantifying Model Accuracy with Rain Impairment Again ................. 61

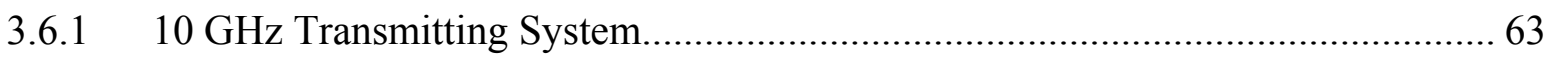

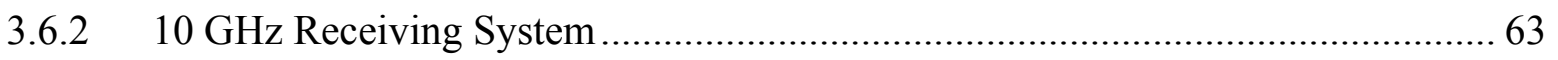

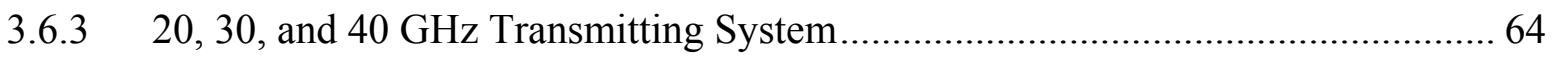

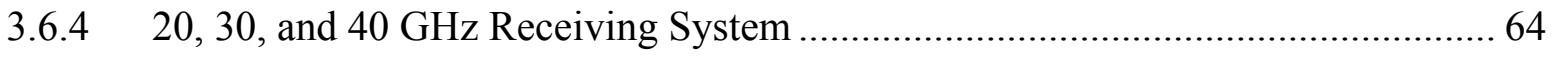




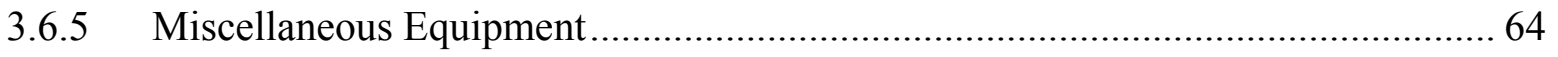

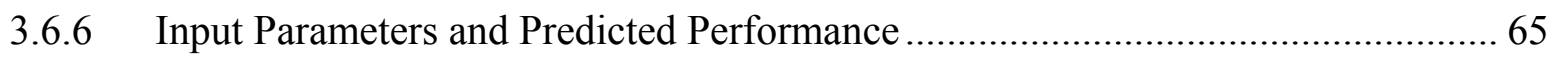

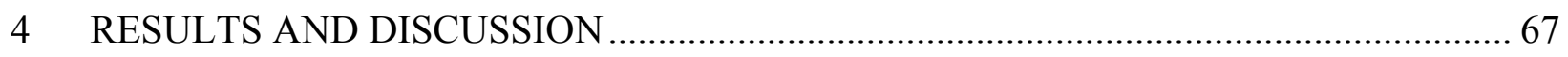

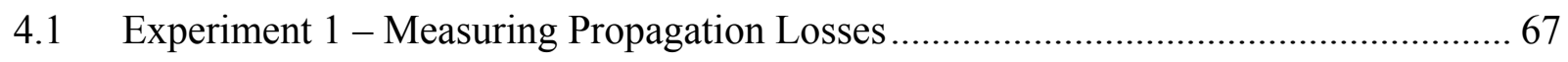

4.2 Experiment 2 - Quantifying Model Accuracy in an Anechoic Chamber ....................... 74

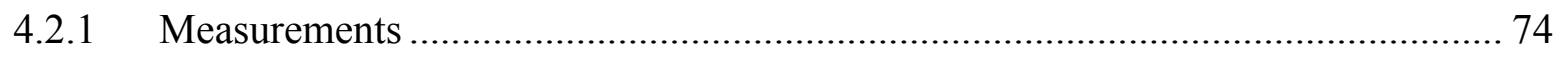

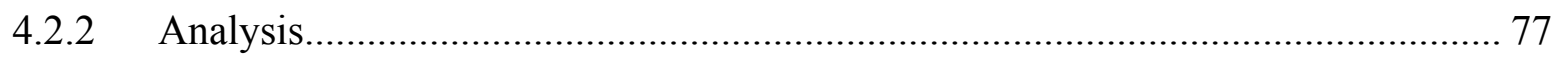

4.3 Experiment 3 - Quantifying Model Accuracy with Rain Impairment ........................... 82

4.4 Experiment 4 - Quantifying Model Accuracy with Rain Impairment Again.................. 86

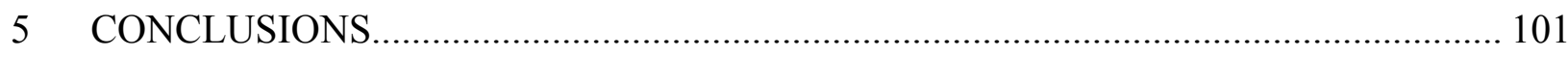

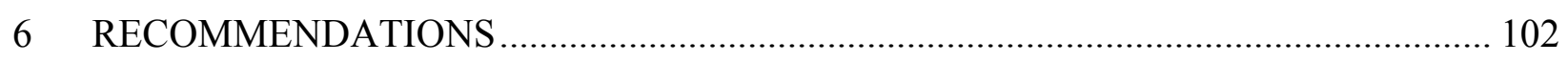

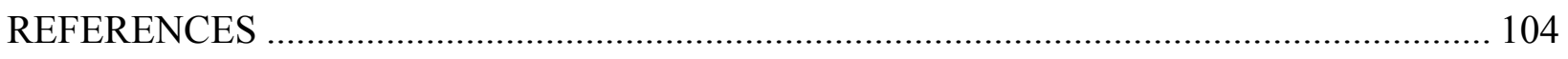

LIST OF SYMBOLS, ABBREVIATIONS AND ACRONYMS.............................................. 106 


\section{LIST OF FIGURES}

Figure 1. Paramagnetic Dipole Moments Aligning With the Applied Magnetic Field ................ 9

Figure 2. Specific Attenuation Due to Gaseous Absorption From 0 - 1000 GHz.................... 11

Figure 3. Specific Attenuation Due to Water Vapor and Dry Air ........................................... 14

Figure 4. The Mie Solution Scattering Phenomenon........................................................ 16

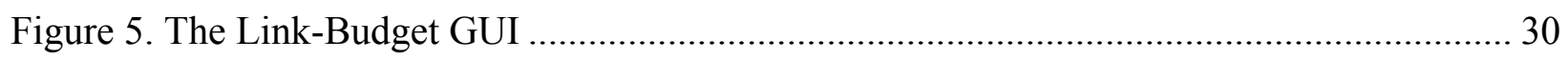

Figure 6. (a) The Channel Specification Panel (Left) Is What The User Normally Sees On The Main GUI; (b) The Pop-up Menu (Right) Is What The User Sees When The "Parameters" Button Has Been Selected ................................................................ 31

Figure 7. (a) The Site Location Panel (Left) Is What The User Normally Sees On The Main GUI;

(b) The Pop-up Menu (Right) Is What The User Sees When The "Coordinates" Button Has Been Selected. 33

Figure 8. (a) The Atmosphere Panel (Left) Is What The User Normally Sees On The Main GUI;

(b) The Pop-up Menu (Right) Is What The User Sees When The "Parameters" Button

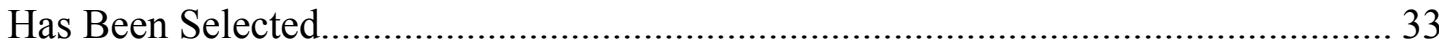

Figure 9. (a)The Transmitting Panel (Left) Is What The User Normally Sees On The Main GUI;

(b) The Pop-up Menu (Right) Is What The User Sees When The "System Parameters" Button Has Been Selected; Note That The Amplifier Button Is Not Selected In This Case. 34

Figure 10. (a) The Receiving Panel (Left) Is What The User Normally Sees On The Main GUI;

(b) The Pop-up Menu (Right) Is What The User Sees When The "System Parameters" Button Has Been Selected; Note That The Amplifier Button Was Selected In This Case 35

Figure 11. Excess Attenuation Panel 35

Figure 12. Dust Storms Visibility Level Drop-Down Menu; Note That The Lower The Visibility, The More Severe The Attenuation Is Expected To Be ........................................... 36

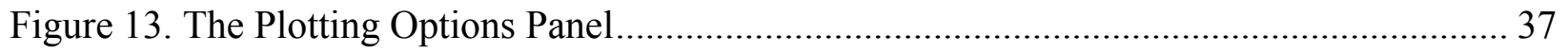

Figure 14. Specific Attenuation Panel Which Only Lists The Active Attenuators In The Link-Budget Calculation 38

Figure 15. Output Statistics Panel Which Is Filled In Every Time A Link-Budget Calculation Is Run. 38

Approved for Public Release; Distribution is Unlimited. 
Figure 16. Typical Warning Box That Would Pop-up When Either No Entries Were Saved Or The Data Fields Were Not Correctly Filled Out.... 39

Figure 17. Typical Error Box That Would Pop-up When Program Execution Is Attempted

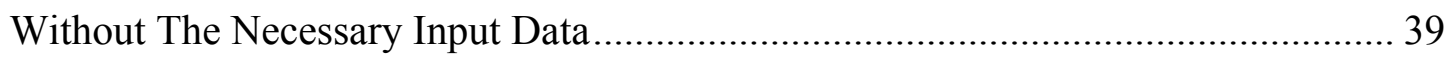

Figure 18. An Overview of the First Propagation Experiment at $10 \mathrm{GHz}$................................. 43

Figure 19. Actual Footage of the First Experiment at $10 \mathrm{GHz}$................................................... 44

Figure 20. Actual Footage of the Experiment at 10, 20, 30, and $40 \mathrm{GHz}$ within the Anechoic

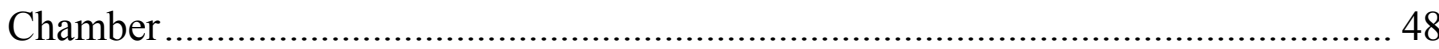

Figure 21. Top View Animation of the Rain Simulator Exemplifying the Uniform Distribution of

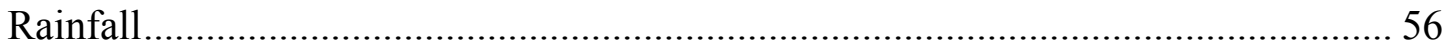

Figure 22. The Rainfall Experimental Setup; Note the Appreciable Height of the Water Distribution Lines and the Relatively Clear LOS Path ................................................... 62

Figure 23. Measured Received Power at $10 \mathrm{GHz}$ for a Channel Length of $46 \mathrm{~m}$.......................... 68

Figure 24. Measured Received Power at $10 \mathrm{GHz}$ for a Transmit Power of $23 \mathrm{dBm}$..................... 68

Figure 25. Measured Received Power at $10 \mathrm{GHz}$ for a Transmit Power of $20 \mathrm{dBm}$..................... 69

Figure 26. Measured Received Power at $10 \mathrm{GHz}$ for a Transmit Power of $17 \mathrm{dBm}$..................... 69

Figure 27. Measured Received Power at $10 \mathrm{GHz}$ for a Transmit Power of $14 \mathrm{dBm}$.................... 70

Figure 28. Measured Received Power at $10 \mathrm{GHz}$ for a Transmit Power of $11 \mathrm{dBm}$..................... 70

Figure 29. The Predicted Received Power Compared Against the Measured Values................... 71

Figure 30. Data Comparison Between the Normalized Data of the Predicted and Measured

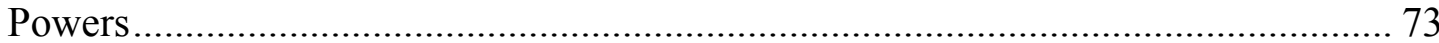

Figure 31. Plot of the Raw Output Data with Maximum Power Received at $10 \mathrm{GHz}$................ 75

Figure 32. Plot of the Raw Output Data with Maximum Power Received at $20 \mathrm{GHz}$................. 76

Figure 33. Plot of the Raw Output Data with Maximum Power Received at $30 \mathrm{GHz}$................. 76

Figure 34. Plot of the Raw Output Data With Maximum Power Received at $40 \mathrm{GHz}$............... 77

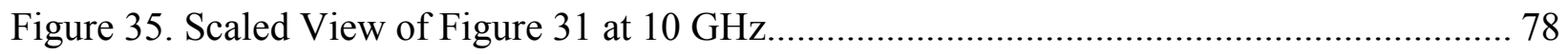

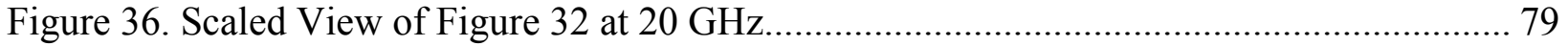

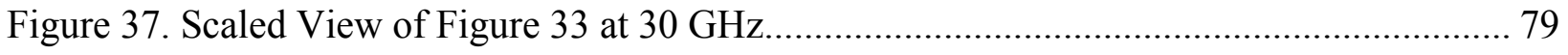

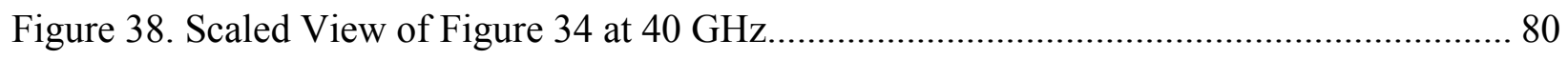

Figure 39. Comparing Measurement Spreads and Model Accuracy at the Four Examined Frequencies

Approved for Public Release; Distribution is Unlimited. 
Figure 40. Analyzing the Measurements Prior to Rainfall and Comparing Them to the Predicted Value 83

Figure 41. Analyzing the Measurements with Rainfall and Comparing Them to the Predicted Value 84

Figure 42. Analyzing the Measurements Post-Rainfall and Comparing Them to the Predicted Value 84

Figure 43. Average Data Sets for Before, During, and After Rainfall for $10 \mathrm{GHz}$. 87

Figure 44. Average Data Set for Before Rainfall for $10 \mathrm{GHz}$ (Showing Measurement Values (Blue 'o') and Predicted Values (Red 'x')). 87

Figure 45. Average Data Set for During Rainfall for $10 \mathrm{GHz}$ (Showing Measurement Values (Blue 'o') and Predicted Values (Red ' $\mathrm{x}$ ')) 88

Figure 46. Average Data Sets for Before, During, and After Rainfall for $20 \mathrm{GHz}$ 88

Figure 47. Average Data Set Prior to Rainfall at $20 \mathrm{GHz}$ (Showing Measurement Values (Blue 'o') and Predicted Values (Red ' $\mathrm{x}$ ')) 89

Figure 48. Average Data Set for During Rainfall for $20 \mathrm{GHz}$ (Showing Measurement Values (Blue 'o') and Predicted Values (Red ' $\mathrm{x}$ ')). 89

Figure 49. Average Data Set for Post-Rainfall for $20 \mathrm{GHz}$ (Showing Measurement Values (Blue 'o') and Predicted Values (Red ' $\mathrm{x}$ ')) ..... 90

Figure 50. Average Data Sets for Before, During, and After Rainfall for $30 \mathrm{GHz}$. 90

Figure 51. Average Data Set For Before Rainfall for $30 \mathrm{GHz}$ (Showing Measurement Values (Blue 'o') and Predicted Values (Red 'x')).... 91

Figure 52. Average Data Set For During Rainfall for $30 \mathrm{GHz}$ (Showing Measurement Values (Blue 'o') and Predicted Values (Red ' $\mathrm{x}$ ')). 91

Figure 53. Average Data Set For Post-Rainfall for $30 \mathrm{GHz}$ (Showing Measurement Values (Blue 'o') and Predicted Values (Red 'x')) 92

Figure 54. Average Data Sets For Before, During, and After Rainfall for $40 \mathrm{GHz}$ 92

Figure 55. Average Data Set for Before Rainfall for $40 \mathrm{GHz}$ (Showing Measurement Values (Blue 'o') and Predicted Values (Red ' $\mathrm{x}$ ')). 93

Figure 56. Average Data Set for During Rainfall for $40 \mathrm{GHz}$ (Showing Measurement Values (Blue 'o') and Predicted Values (Red 'x')) 93 
Figure 57. Average Data Set For Post-Rainfall for $40 \mathrm{GHz}$ (Showing Measurement Values (Blue 'o') and Predicted Values (Red 'x')) 94

Approved for Public Release; Distribution is Unlimited. 


\section{LIST OF TABLES}

Table 1. Input Parameters For A Sample Link-Budget Calculation At $10 \mathrm{GHz}$......................... 40

Table 2. Sample Calculation At 10 GHz Performed By The Coupled Model................................ 41

Table 3. Input Parameters and Link-Budget Calculations for the First Experiment ..................... 46

Table 4. Output Data From the Link-Budget Model for the First Experiment.............................. 46

Table 5. Output Data Post Data Analysis Discussion.................................................................. 47

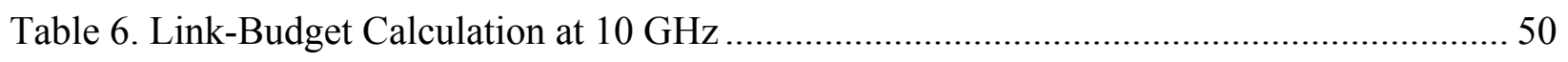

Table 7. Input Parameters For Link-Budget Calculations At 20-40 GHz ................................... 51

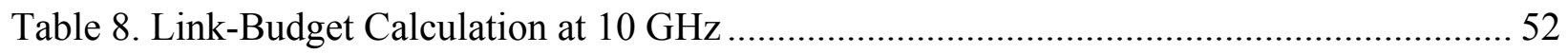

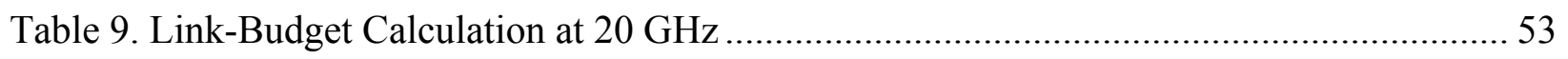

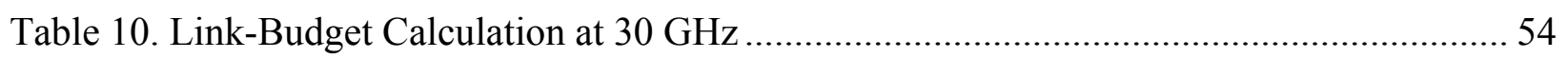

Table 11. Link-Budget Calculation at $40 \mathrm{GHz}$................................................................... 55

Table 12. Input Parameters for a Link-Budget Calculation at $10 \mathrm{GHz}$......................................59

Table 13. Link-Budget Calculation at $10 \mathrm{GHz}$ During a Rainfall at $20 \mathrm{~mm}$ /hour......................... 60

Table 14. Link-Budget Calculation at $10 \mathrm{GHz}$ During Clear-Sky Atmosphere .............................. 61

Table 15. Input Parameters for a Link-Budget Calculation at $10 \mathrm{GHz}$...................................... 65

Table 16. Input Parameters for a Link-Budget Calculation at 20, 30, and $40 \mathrm{GHz}$....................... 66

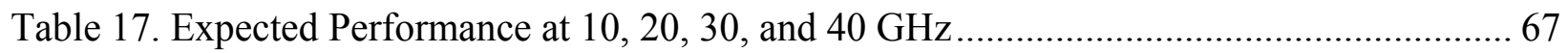

Table 18. Measured Received Power at $10 \mathrm{GHz}$ for a Channel Length of 46m............................. 67

Table 19. The Initial Comparison Between the Measured Results and the Predicted Data .......... 71

Table 20. The Initial Comparison Between the Measured Results and the Predicted Data ......... 74

Table 21. The Measured Power Values at the Primary Frequencies of Interest............................ 75

Table 22. Measured Power Values at the Primary Frequencies of Interest .................................. 77

Table 23. Measured and Predicted Data for Clear-Sky and Rain Impairments............................. 85

Table 24. Statistical Calculations of the Measured Data ................................................................. 86

Table 25. $10 \mathrm{GHz}$ Data Sets Used for Statistical Analysis ....................................................... 95

Table 26. 20 GHz Data Sets Later Used for Statistical Analysis.................................................... 96

Table 27. Simple Table Conveying the Average Measured Power and the Predicted Measured

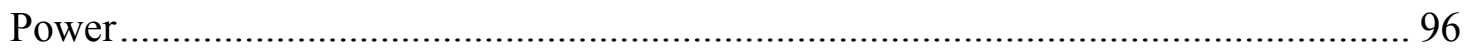

Table 28. 30 GHz Data Sets Later Used for Statistical Analysis.................................................. 97

Table 29. 40 GHz Data Sets Later Used for Statistical Analysis..................................................... 97 
Table 30. Measured Data Comparison to Ensure That Losses Were Indeed Present During

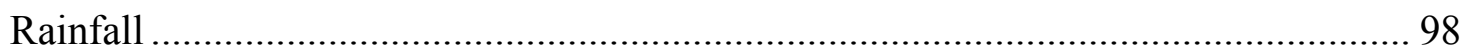

Table 31. Statistical Data For All Frequencies During Rainfall .................................................. 98

Table 32. Statistical Data For All Frequencies During The Clear-Sky Scenario ......................... 99

Table 33. Statistical Data with Correction Factor...................................................................... 100 


\section{ACKNOWLEDGMENTS}

This material is based on research sponsored by Air Force Research Laboratory under agreement number FA9453-13-1-0230. The U.S. Government is authorized to reproduce and distribute reprints for Governmental purposes notwithstanding any copyright notation thereon.

\section{DISCLAIMER}

The views and conclusions contained herein are those of the authors and should not be interpreted as necessarily representing the official policies or endorsements, either expressed or implied, of Air Force Research Laboratory or the U.S. Government. 
(This page intentionally left blank)

Approved for Public Release; Distribution is Unlimited. 


\section{SUMMARY}

The $\mathrm{V}$ and $\mathrm{W}$-bands are becoming of interest to satellite designers due to the large availability of bandwidth and the potential multi-gigabit data rates. Implementation of a satellite communication channel at these frequencies has not been documented in literature, and commercial wireless services at these frequency bands are undeveloped. Furthermore, millimeter waves are affected much more by atmospheric attenuation, with rain being the most detrimental and uncertain. Predictive models of rain attenuation claim some degree of accuracy up to 55 $\mathrm{GHz}$, although they are primarily based on measurements at $40 \mathrm{GHz}$ and below. This project focuses on the analysis and verification of propagation models for electromagnetic waves through atmosphere at millimeter wavelengths. Tests are performed below $40 \mathrm{GHz}$, but as a precursor for testing below $90 \mathrm{GHz}$.

\section{INTRODUCTION}

\subsection{Motivation}

There are many advantages in exploiting higher frequencies, but the two that stand out are: (1) availability of larger bandwidths, and (2) the decrease in antenna size. Larger bandwidths are especially attractive for multimedia services, which use larger bandwidths to obtain higher data rates for their users. Additionally, smaller antennas are appealing to satellite system designers due to their higher directivity, portability, and lower cost. Conveniently, the Federal Communications Commission (FCC) of the United States of America decided to allocate a portion of the "millimeter-wave" spectrum in $\mathrm{V}$ and $\mathrm{W}$-bands to a shared development and utilization between both government and non-government entities for satellite communication. Specifically, the frequencies assigned for satellite communication are $71-76 \mathrm{GHz}, 81-86 \mathrm{GHz}$, and 92-95 GHz. This allocation of frequency allows for the development of the Extremely High Frequency (EHF) regime. Up to this point in time, satellite communication services in these bands are non-existent.

Although there are some prominent advantages in the utilization of EHF bands, there also exist many propagation impairments that lower frequency bands do not experience. The attenuation contributions of clouds and fog, atmospheric gaseous absorption, rain, scintillations, and signal depolarization are some of the major factors that make design and implementation at

Approved for Public Release; Distribution is Unlimited. 
EHF challenging. Moreover, few propagation research activities have conducted at frequencies above $50 \mathrm{GHz}$, with only a handful being carried out over the last few years. With the lack of work in the EHF band comes the need to perform extensive research into the detrimental propagation impairments that a potential radio link could encounter.

The radio communication Sector of the International Telecommunications Union (ITUR) has presented many validated atmospheric models to aid in the design of radio frequency (RF) channels. Although these models are accepted by the RF community, it is imperative to ensure that they are capable at lower frequencies before proceeding to the more challenging EHF regime. The rain models supported by the ITU-R claim validity up to about $55 \mathrm{GHz}$, even though they are primarily based off of $40 \mathrm{GHz}$ measurements and significantly lack data above $40 \mathrm{GHz}$. Needless to say, development of a radio link model at frequencies well above current model limitations would be highly beneficial to high-frequency applications.

In order to perform any type of experimental research at such frequencies, it is imperative to design a stable link that can handle at least predicted impairments. Historically, characterization and model validation has been conducted via the use of space-beacon and a distributed network of ground terminals. This approach allows for statistical understanding of the interaction between radio signals and environmental impairments such as rain, clouds and dust (although rain is the most typical impairment to be monitored). Although this method is very useful, it is extremely expensive to implement $(>\$ 1 \mathrm{M})$ and could be high-risk for the $\mathrm{V}$ and $\mathrm{W}$ frequencies, since the outcome would be unknown above standard model limitations. Prior to investing funds into a risky high-frequency communications link, it would be extremely beneficial for practical measurements to be taken utilizing a terrestrial link. This link will be a cost-efficient way of testing current propagation models, and developing.

A stable terrestrial communications link would meet this need. This alternative method would be much more cost effective, while still providing meaningful results. Development of a successful link could allow for first-ever terrestrial validation of rain and depolarization models in the $\mathrm{Q} / \mathrm{V}$-bands. Furthermore, current models could be compared against actual data, and could be further improved. Data collection and rain and depolarization model verification could lead to highly significant scientific contributions in atmospheric propagation. 


\section{$2.2 \quad$ Literature Review}

The book, Satellite-to-Ground Radiowave Propagation by J.E. Allnutt [1], is a comprehensive guide intended for satellite-to-ground system designers when working with frequencies above $1 \mathrm{GHz}$. In this text, the author covers a wide range of propagation impairments to be expected when designing a communications link at high frequencies. The text provides step-by-step procedures for modeling the natural and weather-induced attenuations that a signal above $1 \mathrm{GHz}$ would expect. Though the models were primarily taken from the ITU-R, this book compiles all of them into an easily understandable read.

This book was the overall primary reference for the design of the link-budget and impairments coupled model in this work. Additionally, the theoretical background, which is not provided by the ITU-R, was discussed in a fair amount preceding each model's presentation. Though the theory is not necessary to develop a model, it is useful in understanding the sources of attenuation that the radiowave will experience.

For example, the models provided by the ITU-R [2,3] and presented in [1] surround the weather impairment of rain. Had the model been followed without theoretical background, then one would expect that attenuation due to rain would be a simple linear relationship with frequency. This is not the case. As the wavelength of the signal becomes comparable in size to a hydrometeor (precipitation product such as rain), the signal losses can no longer be attributed to primarily scattering as the Rayleigh model would indicate. The signal will be more heavily absorbed, and losses will increase substantially. This is only one example where the theoretical knowledge provided in [1] has proved useful.

In [4], efforts similar to the overall goal of this work are presented. Specifically, efforts in [4] present the plan to implement a $96 \mathrm{GHz}$ terrestrial communications link spanning approximately $7.4 \mathrm{~km}$. The group working on this communications link is based out of Italy and is the only other known group to be investigating a terrestrial W-band communications link. In addition to the discussion of the potential link, the transmitting and receiving system architectures are described for the W-band.

The overall goals in [4] were to implement a terrestrial link in the W-band, monitor propagation impairments, and ultimately characterize the $94-96 \mathrm{GHz}$ frequency range in order to be implemented in future satellites. This goal is similar to the overall mission of this thesis in 
that frequency characterization in the $\mathrm{V}$ and $\mathrm{W}$-bands are desired to implement high-frequency satellite communications. Additionally, some of the propagation models utilized in [4] and in this thesis are from the ITU-R.

In the work presented in this thesis though, an alternative method to measuring rain impairments is presented. This method allows for rain simulation to occur whenever measurements are desired. This capability allows for major measurement flexibility in that rain attenuation can be characterized along with other impairments such as scintillations and temperature variance since the simulator can be activated at any time.

Though the sources listed previously have direct connections to the work performed here, it is useful to discuss the current standards of characterizing propagation links. Prior to the work here and the work discussed above, characterization and model validation of rain effects on radiowaves have been conducted through the use of space beacons and ground distributed networks. Well known campaigns are those of ACTS, OLYMPUS, and ITALSAT F1.

The Advanced Communications Technology Satellite (ACTS) was the first all-digital, gigabit-capacity communications satellite [5]. The ACTS was launched in 1993 and collected data all the way up until 2004 when it was decommissioned [5]. The ACTS measured over 35 station-years of data at $\mathrm{K}$ and Ka frequency bands over the United States. The data collected provided a major influence upon the ITU-R rain models [2] and yielded regional precipitation characterization [5].The OLYMPUS collected approximately 15 station-years of data at 12, 20, and $30 \mathrm{GHz}$, that characterized several regions for rainfall. The ITALSAT F1 characterized the 20,40 , and $50 \mathrm{GHz}$ frequency channels with about 10 years of data collected at 15 sites spread throughout Europe [2].

From these experiments, it is evident that satellite channel characterization is not normally performed with a terrestrial link. These are some of the systems that have provided the fundamental background for the work in this thesis. Though the missions above all provided substantial data for multiple sites, the $\mathrm{V}$ and $\mathrm{W}$-band frequencies provide a much more challenging approach, since the losses that occur here are greatly different than anything experienced before. Ultimately, there could be a great risk in launching a satellite with $\mathrm{V}$ and $\mathrm{W}$ band capabilities if the current prediction methods are used in the system design, especially considering that the models are based upon $40 \mathrm{GHz}$ data and below. The work here would reduce

Approved for Public Release; Distribution is Unlimited. 
the risk, and assist in greater planning techniques to mitigate the characterized losses in the Wband.

\subsection{Scope of Effort}

It is essential to the development of satellite and earthbound links that an accurate model be created to have a rough expectation of how the terrestrial link might perform. In this research project / report, the development of a detailed link-budget analysis tool is discussed addressing both the advantages and limitations of the models used. Creating an analysis tool is practically useless if there are no data supporting veracity. Therefore, the model was experimentally validated by comparing several measurements with model predictions, which are explained in detail throughout this work. This validation is imperative to prove system accuracy and provide reason to utilize the model as a baseline for the $\mathrm{V}$ and $\mathrm{W}$-band experiments.

Since long-distance propagation links at 70 and $80 \mathrm{GHz}$ are highly unexplored, initial experiments at the well-known frequencies were conducted. Specifically, propagation at 10, 20, 30, and $40 \mathrm{GHz}$ were modeled and compared against measured data. Although it was not expected for the attenuation contributions of individual impairments to linearly scale to the higher $\mathrm{V}$ and $\mathrm{W}$-bands, experiments at $40 \mathrm{GHz}$ and below would validate the current ITU-R models and measure their accuracy. In particular, proving the models' accuracy during heavy rain would be ideal in planning $\mathrm{V}$ and $\mathrm{W}$-band links due to their high susceptibility to precipitation.

During the course of this work, many models account for rain, clouds, fog, dirt, were utilized in the development of a link-budget Graphical User Interface (GUI). Many of these models were from the ITU-R, while a handful of others were from various other sources. This GUI was primarily created to provide timely model and measurement comparison. Additionally, the functionality of the GUI extends to adding atmospheric impairments to the link in which the user could specify a "worst-case" scenario when planning a link.

After the development of the predictive model, several experiments were conducted to verify the models accuracy. The first experiment was conducted at $10 \mathrm{GHz}$ and had a primary goal of transmitting and receiving a signal across a channel in a practical situation. The secondary goal was to compare the measured results with the designed propagation model and 
determine the error. The channel was specifically setup between the roofs of University of New Mexico (UNM)'s Electrical \& Computer Engineering and Centennial Engineering Buildings.

The second set of experiments was conducted in which the test system was characterized in an anechoic chamber. Measurements were taken at $10 \mathrm{GHz}, 20 \mathrm{GHz}, 30 \mathrm{GHz}$, and $40 \mathrm{GHz}$. This allowed cleaner measurements for model validations, and permitted a better understanding of test equipment performance prior to later out-door testing.

The third set of experiments was conducted using a rain-simulator to measure the propagation losses across before, during, and after rain simulation at $10 \mathrm{GHz}$. The hope was that noticeable losses would be measured and the results allow validation and verification of the linkbudget model.

The fourth set of experiments was conducted using a rain-simulator to measure the propagation losses at higher frequency $(20 \mathrm{GHz}, 30 \mathrm{GHz}$, and $40 \mathrm{GHz}$ ). Data was used to assess model accuracy and experiment uncertainty.

Ultimately, the work done here is intended to provide the necessary procedure to follow when designing higher-frequency communication links. The terrestrial data collected during the rain experiments will be highly beneficial to the design and execution of $\mathrm{V}$ and $\mathrm{Q}$ band communication links. Additionally, the challenges that occurred during this initial experimentation will be monitored more closely, and will allow the future work to continue more smoothly.

\section{METHODS, ASSUMPTONS, AND PROCEDURES}

\subsection{Coupled Model Development and Link Analysis}

As mentioned previously, the EHF band experiences significantly more attenuation than lower frequency bands while propagating. This is true under both clear and inclement weather conditions. During clear conditions, radio waves are impacted primarily by attenuation sources such multipath, scintillation, gaseous absorption, reflection, and others. Radio waves are even more vulnerable to inclement weather than they are to clear conditions. This is partly due to the fact that as frequency increases, the wavelength becomes comparable in size to the particles with which the wave interacts. This decrease in wavelength makes the waves particularly susceptible to loss from fog, clouds, dirt, and especially rain and snow.

Approved for Public Release; Distribution is Unlimited. 
The Radio-communication Sector of the International Telecommunications Union (ITU$\mathrm{R}$ ) is one of the world leaders in ensuring rational, equitable, efficient and economical use of the radio-frequency by all radio-communication services. The ITU-R carries out studies and adopts recommendations on radio communication matters. The adopted recommendations come from research conducted all over the world. Essentially, the propagation models are chosen based on both comparison to measured data and agreement with electromagnetic theory. Therefore, the models when used within the specified limitations should be accurate with the specified accuracy.

The use of propagation models is vital when planning a communication link using millimeter-wave frequencies. They are even more necessary when planning a communication link in the Extremely High Frequency (EHF) bands. Therefore, it was believed to be necessary to investigate the current attenuation models to determine if EHF propagation was even possible at any appreciable distance. Based on the primary attenuation effects that impact $\mathrm{V}$ and $\mathrm{W}$-band frequency bands, the decision was to further investigate the models concerning effects of rain, scintillation, gaseous absorption, dust storms, clouds and fog. The details of the current models describing these effects are explained in the subsequent sections.

\subsubsection{Attenuation Effects}

\subsubsection{Gaseous Absorption and Water Vapor}

During atmospheric propagation, an electromagnetic wave interacts with many different earth gasses. Although interactions with many of these molecules will result in negligible losses, interactions with some will have an extremely noticeable impact. This is due to the absorption of the electromagnetic radiation. In electromagnetic theory, absorption of electromagnetic radiation is the process in which the energy of an electromagnetic wave is taken, or absorbed, by a given medium. Within the absorber, the electromagnetic energy that is taken is converted to an equivalent amount of energy, such as thermal or other forms of molecular energy. This reduction

of electromagnetic radiation intensity is attenuation that the wave experiences when it is incident upon a lossy material and occurs with all mediums.

The refractive index of a material describes how an electromagnetic wave, or other forms of radiation, propagates through that medium. This is given by 


$$
n=\frac{c}{v},
$$

where $c$ is the speed of light in a vacuum, and $v$ is the speed of light in the medium. If a material is opaque, the refractive index is a complex number. The real part of this complex number represents refraction, whereas the imaginary part signifies absorption. The complex refractive index is represented as

$$
\dot{n}=n^{\prime}+j n^{\prime \prime},
$$

where $n$ ' is the real, or refractive component, and $n$ ', is the imaginary, or absorptive part of the index of refraction. The absorptive component, $n$ ', can be inserted into the equation of the electric field for a plane wave traveling in the $z$ direction in order to determine intensity of absorption. The time-harmonic form of the electric field is given by

$$
\mathcal{E}(z ; t)=\operatorname{Re}\left[\mathbf{E}(z ; t) e^{j(k z-\omega t)}\right],
$$

where $\omega$ is defined as the radian frequency as $2 \pi f, z$ is the position, $t$ is the propagation time, and $k$ is the wave number defined as $\frac{2 \pi n}{\lambda}$. Further examining the wave number expression, it can be seen that it is dependent on the refractive index, $n$, and the wave length of the transmitted wave. Inserting the complex refractive index into the electric field expression yields:

$$
\begin{gathered}
\mathcal{E}(z ; t)=\operatorname{Re}\left[\mathbf{E}(z ; t) e^{j\left(\frac{2 \pi\left(n^{\prime}+j n^{\prime \prime}\right)}{\lambda} z-\omega t\right)}\right]=\operatorname{Re}\left[\mathbf{E}(z ; t) e^{j\left(\left(\frac{2 \pi n^{\prime}}{\lambda}+j \frac{2 \pi n^{\prime \prime}}{\lambda}\right) z-\omega t\right)}\right] \\
=\operatorname{Re}\left[\mathbf{E}(z ; t) e^{-\frac{2 \pi n^{\prime \prime}}{\lambda}} e^{j\left(\frac{2 \pi n^{\prime}}{\lambda} z-\omega t\right)}\right]=e^{-\frac{2 \pi n^{\prime \prime}}{\lambda} z} \operatorname{Re}\left[\mathbf{E}(z ; t) e^{j\left(\frac{2 \pi n^{\prime}}{\lambda} z-\omega t\right)}\right] .
\end{gathered}
$$

The exponential expression in the front conveys that the $n$ " results in exponential decay. Taking the magnitude of the electric field results in

$$
e^{-\frac{4 \pi n^{\prime \prime}}{\lambda} z}|\mathbf{E}|^{2}
$$

The square of the electric field is proportional to the radiation intensity, and results in the attenuation component increasing by a factor of two. According to the Beer-Lambert law, this term is defined as the absorption coefficient. When $n$ '" is positive, radiation is absorbed. If it is zero, then the material is transparent to the wave.

Similarly, the index of refraction is related to the relative permittivity and permeability of a material through 


$$
\dot{n}^{2}=\mu_{R} \varepsilon_{R},
$$

where $\mu_{R}$ and $\varepsilon_{R}$ are defined as the relative permeability and permittivity respectively. These variables are convenient in the fact that the material's nature can be described by observation and simple calculations. The nature of interest is often the mediums susceptibility to the electric and magnetic field.

A material is considered to be a polar material if, in the absence of an applied electric field, the material possesses randomly oriented permanent dipole moments [6]. Similarly, magnetic materials are those that exhibit magnetic polarization when they are subjected to an applied magnetic field. Basically, when an applied field is present, the dipoles within a material will align with the field if it is magnetic or polar. This is conveyed in Figure 1.

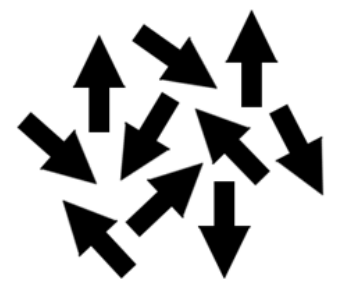

Applied Magnetic Field Absent

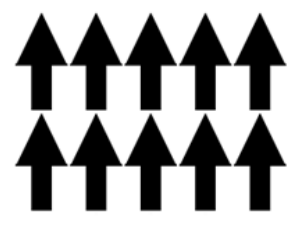

Applied Magnetic Field Present

\section{Figure 1. Paramagnetic Dipole Moments Aligning With the Applied Magnetic Field}

Magnetic and polar molecular compounds exhibit significantly more losses than other molecules due to their magnetic moment rapidly fluctuating. Essentially, the applied field is fluctuating in time (e.g. cosine and sine waves). This fluctuation results in the constant reorientation of magnetic dipole moments to align with the applied field. As frequency increases, the moments will need to align more quickly. When the frequency becomes too high, the moments cannot orient quickly enough, and fall into a "limbo" state in which they become closer to static. This, in turn, translates to higher losses.

During propagation typically below $70 \mathrm{GHz}$, the compounds of most interest are those of oxygen gas and gaseous water vapor [1]. Oxygen happens to be a magnetic molecule, which has the properties as stated previously. However it is only paramagnetic, meaning that it is only 
slightly affected by an applied magnetic field. Oxygen has a magnetic susceptibility, $\chi_{m}$, of $209 \times 10^{-8}$. This is related to the much more common relative permeability term by

$$
\mu_{R}=1+\chi_{m} \text {. }
$$

Although the susceptibility term appears to be almost negligible, it still results in oxygen experiencing resonant absorption at certain frequencies. Note that $60 \mathrm{GHz}$ happens to be one of the major resonant frequencies of oxygen, while $80 \mathrm{GHz}$ is a local minima in the higher frequency range.

The ITU-R has two recommendations to model attenuation due to gaseous absorption that are contained in ITU-R P.676-9 [7]. The first recommendation listed is for general applications and is considered accurate for frequencies up to $1000 \mathrm{GHz}$ [7]. However, this method is computationally intensive and the level of accuracy it provides is not usually required. The second recommendation, specific attenuation, is meant for approximate estimates of gaseous attenuation that covers the frequency range of 1 to $350 \mathrm{GHz}$ [7]. Both of these methods will be discussed since both were utilized throughout this work.

Both of the prediction methods in the ITU-R P.676-9 recommendation achieve their specific and standard attenuation calculations accuracy with knowledge of atmospheric data and channel specifications. Specifically, the models call for frequency, pressure, temperature, channel length, latitude, season, receiver elevation, and water vapor density as provided by ITU$\mathrm{R} 836$ [8]. This recommendation utilizes years of past data to determine average water vapor density profiles solely based on location and season.

The more complex calculation of the ITU-R P.676-9 recommendation obtains the specific attenuation attributed by water vapor and dry air from knowledge of the imaginary part of the complex refractivity [7]. The expected values from this model are shown in Figure 2.The dry air portion incorporates oxygen, pressure-induced nitrogen, and non-resonant Debye attenuation. Ultimately, this model calculates the frequency dependent refractivity term from the variables stated above, and calculates the "dry" and "wet" specific attenuation terms as follows.

$$
\gamma=\gamma_{\mathrm{o}}+\gamma_{\mathrm{w}}=0.1820 \mathrm{fN}^{\prime \prime}(\mathrm{f}) \text {. }
$$

For the purpose of this work, the propagation channel will be terrestrial and the attenuation contributed by gaseous attenuation is simply a multiplication of specific attenuation and channel length.

Approved for Public Release; Distribution is Unlimited. 


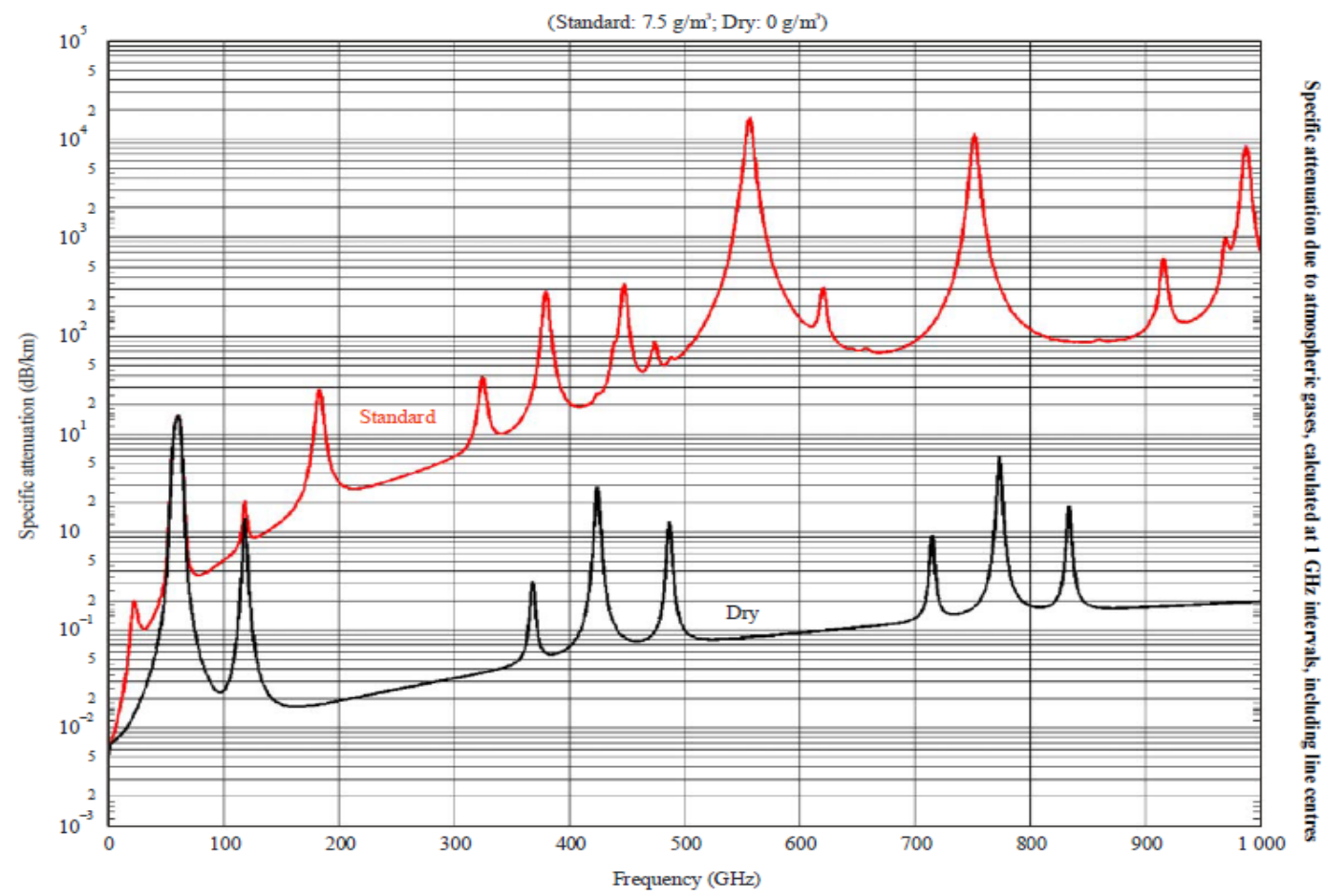

Figure 2. Specific Attenuation Due to Gaseous Absorption From 0 - 1000 GHz

The second method, although seemingly more invasive, is much easier to implement and utilize for quick estimates. This formula calculates the specific attenuation due to water vapor and dry air at an altitude of $10 \mathrm{~km}$ [7]. An extension of this algorithm to variable altitudes will be briefly discussed at the end of this section. The algorithm has proven to agree with more accurate calculations of gaseous absorption within an "average of about $\pm 10 \%$ at frequencies removed from the centers of major absorption lines" [7]. Note that the absorption lines that the ITU-R is referring to are the spikes in molecular absorption. This approximates out to a difference of 0.1 $\mathrm{dB} / \mathrm{km}$ from the more accurate and intense calculations.

For dry air with $f \leq 54 \mathrm{GHz}$, the attenuation can be modeled as:

$$
\gamma_{o}=\left[\frac{7.2 r_{t}^{2.8}}{f^{2}+.34 r_{p}^{2} r_{t}^{1,6}}+\frac{.62 \xi_{3}}{(54-f)^{1.16 \xi_{1}+.83 \xi_{2}}}\right] f^{2} r_{p}^{2} \times 10^{-3}
$$

For $54<f \leq 60 \mathrm{GHz}$, the attenuation can be modeled as: 


$$
\gamma_{o}=\exp \left[\begin{array}{c}
\frac{\ln \gamma_{54}}{24}(f-58)(f-60)-\frac{\ln \gamma_{58}}{8}(f-54)(f-60) \\
+\frac{\ln \gamma_{60}}{12}(f-54)(f-58)
\end{array}\right]
$$

For $60<f \leq 62 \mathrm{GHz}$, the attenuation can be modeled as:

$$
\gamma_{o}=\gamma_{60}+\left(\gamma_{62}-\gamma_{60}\right) \frac{f-60}{2}
$$

For $62<f \leq 66 \mathrm{GHz}$, the attenuation can be modeled as:

$$
\gamma_{o}=\exp \left[\begin{array}{c}
\frac{\ln \gamma_{62}}{8}(f-64)(f-66)-\frac{\ln \gamma_{64}}{4}(f-62)(f-66) \\
+\frac{\ln \gamma_{66}}{8}(f-62)(f-64)
\end{array}\right]
$$

For $66<f \leq 120 \mathrm{GHz}$, the attenuation can be modeled as:

$$
\gamma_{o}=\left\{\begin{array}{c}
3.03 \times 10^{-4} r_{t}^{3.5}+\frac{.283 r_{t}^{3.8}}{(f-118.75)^{2}+2.91 r_{p}^{2} r_{t}^{1.6}} \\
+\frac{.502 \xi_{6}\left[1-.0163 \xi_{7}(f-66)\right]}{(54-f)^{1.16 \xi_{1}+.83 \xi_{2}}}
\end{array}\right\} f^{2} r_{p}^{2} \times 10^{-3}
$$

For $120<f \leq 360 \mathrm{GHz}$, the attenuation can be modeled as:

$$
\gamma_{o}=\left[\frac{3.02 \times 10^{-4}}{1+1.9 \times 10^{-5} f^{1.5}}+\frac{.283 r_{t}^{0.3}}{(f-118.75)^{2}+2.91 r_{p}^{2} r_{t}^{1.6}}\right] f^{2} r_{p}^{2} r_{t}^{3.5} \times 10^{-3}+\delta
$$

where:

$$
\begin{gathered}
\xi_{1}=\varphi\left(r_{p}, r_{t}, .0717,-1.8132, .0156,-1.6515\right) \\
\xi_{2}=\varphi\left(r_{p}, r_{t}, .5146,-4.6368,-.1921,-5.7416\right) \\
\xi_{3}=\varphi\left(r_{p}, r_{t}, .3414,-6.5851, .2130,-8.5854\right) \\
\xi_{4}=\varphi\left(r_{p}, r_{t}, .-0.0112,0.0092,-0.1033,-0.0009\right) \\
\xi_{5}=\varphi\left(r_{p}, r_{t}, 0.2705,-2.7192,-0.3016,-4.1033\right) \\
\xi_{6}=\varphi\left(r_{p}, r_{t}, 0.2445,-5.9191,0.0422,-8.0719\right) \\
\xi_{7}=\varphi\left(r_{p}, r_{t},-0.1833,6.5589,-0.2402,6.131\right) \\
\gamma_{54}=2.192 \varphi\left(r_{p}, r_{t}, 1.8286,-1.9487, .4051,-2.8509\right) \\
\gamma_{58}=12.59 \varphi\left(r_{p}, r_{t}, 1.0045,3.5610,0.15881,1.2834\right) \\
\gamma_{60}=15.0 \varphi\left(r_{p}, r_{t}, 0.003,4.1335,0.0427,1.6088\right) \\
\gamma_{62}=14.28 \varphi\left(r_{p}, r_{t}, 0.9886,3.4176,0.1827,1.3429\right) \\
\gamma_{64}=6.819 \varphi\left(r_{p}, r_{t}, 1.4320,0.6258,0.3177,-0.5914\right)
\end{gathered}
$$




$$
\begin{gathered}
\gamma_{66}=1.908 \varphi\left(r_{p}, r_{t}, 2.0717,-4.1404,0.4910,-4.8718\right) \\
\delta=-0.00306 \varphi\left(r_{p}, r_{t}, 3.211,-14.94,1.583,16.37\right) \\
\left.\varphi\left(r_{p}, r_{t} a, b, c, d\right)=r_{p}^{a} r_{t}^{b} \exp \left[c\left(1-r_{p}\right)+d\left(1-r_{t}\right)\right]\right) \\
r_{p}=\frac{p_{t o t}}{1013} \\
r_{t}=\frac{288}{(273+t)}
\end{gathered}
$$

where $f$ is the frequency in $\mathrm{GHz}, p_{\text {tot }}$ represents total air pressure, $p$ is the pressure in $\mathrm{hPa}$, and $t$ is the mean temperature in $\left({ }^{\circ} \mathrm{C}\right)$ that can be obtained from maps given by the ITU-R.

For water vapor, the attenuation $\gamma_{\mathrm{w}}(\mathrm{dB} / \mathrm{km})$ is given by:

$$
\gamma_{\mathrm{w}}=\left\{\begin{array}{c}
\frac{3.98 \eta_{1} \exp \left[2.23\left(1-r_{t}\right)\right]}{(f-22.235)^{2}+9.42 \eta_{1}^{2}} g(f, 22)+\frac{11.96 \eta_{1} \exp \left[0.7\left(1-r_{t}\right)\right]}{(f-183.31)^{2}+11.14 \eta_{1}^{2}} \\
+\frac{0.081 \eta_{1} \exp \left[6.44\left(1-r_{t}\right)\right]}{(f-321.226)^{2}+6.29 \eta_{1}^{2}}+\frac{3.66 \eta_{1} \exp \left[1.6\left(1-r_{t}\right)\right]}{(f-325.153)^{2}+9.22 \eta_{1}^{2}} \\
+\frac{25.37 \eta_{1} \exp \left[1.09\left(1-r_{t}\right)\right]}{(f-557)^{2}} g(f, 557)+\frac{290 \eta_{1} \exp \left[1.46\left(1-r_{t}\right)\right]}{(f-448)^{2}} \\
+\frac{844.6 \eta_{1} \exp \left[0.99\left(1-r_{t}\right)\right]}{(f-1780)^{2}} g(f, 1780)
\end{array}\right\} f^{2} 2_{t}^{2.5} \rho x 10^{-4}
$$

with:

$$
\begin{gathered}
\eta_{1}=0.955 r_{p} r_{t}^{0.68}+0.006 \rho \\
\eta_{2}=0.735 r_{p} r_{t}^{0.5}+0.0353 r_{t}^{4} \rho \\
g\left(f, f_{i}\right)=1+\left(\frac{f-f_{i}}{f+f_{i}}\right)^{2}
\end{gathered}
$$

where $\rho$ is the water-vapor density $\left(g / \mathrm{m}^{3}\right)$.

The gaseous absorption over a terrestrial (near Earth) link can be calculated easily by using the above models. The total attenuation in $\mathrm{dB}$ due to water and oxygen over a distance $r_{o}$ is given in [1] and [7], and is simply stated as:

$$
\mathrm{A}=\gamma \mathrm{r}_{\mathrm{o}}=\left(\gamma_{\mathrm{o}}+\gamma_{\mathrm{w}}\right) \mathrm{r}_{\mathrm{o}}
$$

Once again, let it be noted that the algorithm discussed was designed around a fixed height of 10 $\mathrm{km}$. In [7], the ITU-R recommends the calculation of an equivalent height, which leads to a total zenith attenuation. Conclusively, gaseous absorption has a strong impact at molecular absorption resonance, and as frequency increases. This can be observed in Figure 3, which depicts the specific attenuation due to dry air and water vapor, and the sum of the two [7]. Ultimately, as the wavelengths of signals get smaller, the impact of atmospheric molecules grows. 


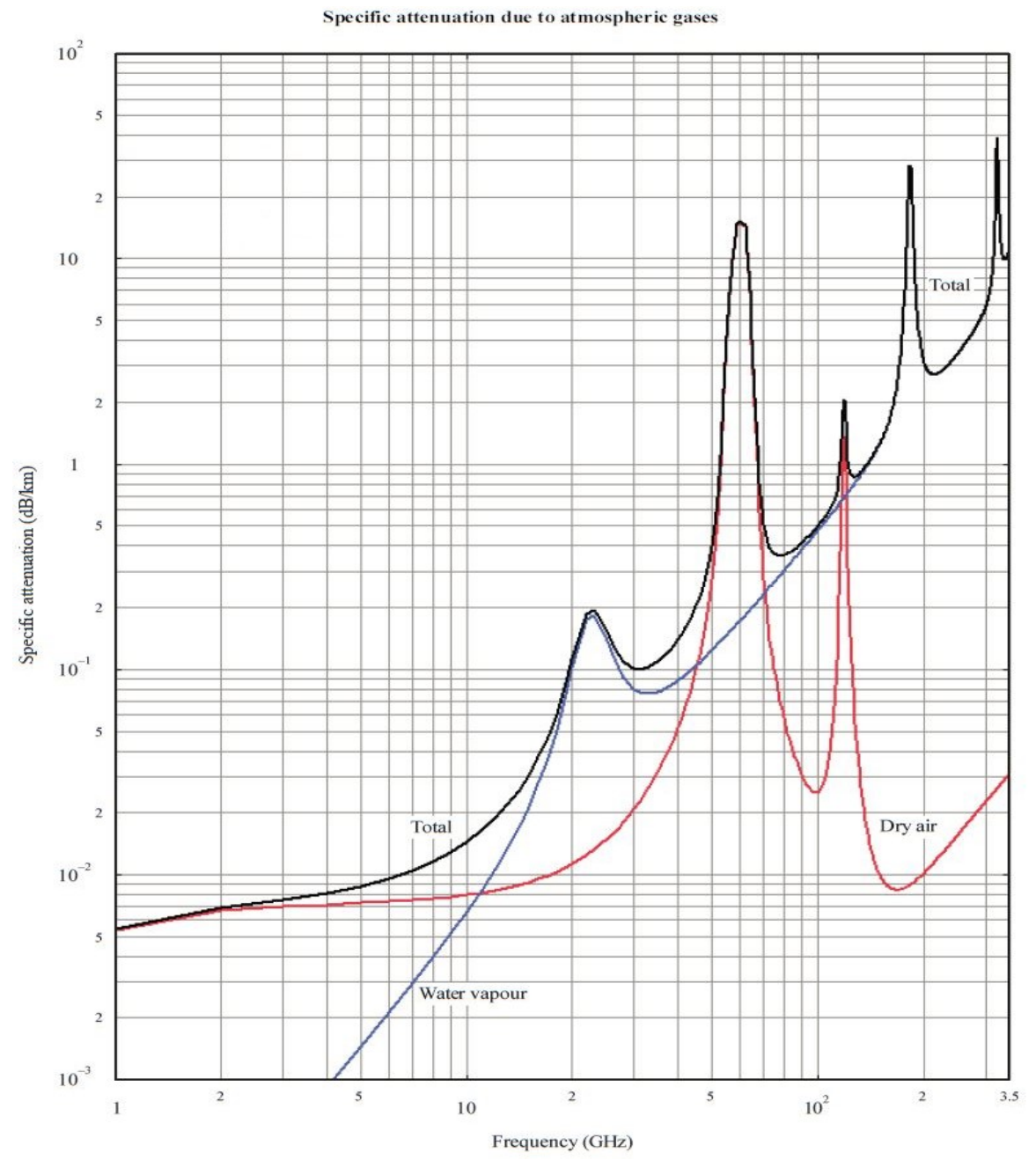

Figure 3. Specific Attenuation Due to Water Vapor and Dry Air

\subsubsection{Rain Losses}

It is well known that rain has a greater impact in the EHF region than at lower frequencies. This is especially true when the wavelength of the signal becomes comparable to the diameter of a rain drop. For example, the wavelength at $80 \mathrm{GHz}$ is $3.75 \mathrm{~mm}$ whereas the diameter of a rain drop ranges from $0.17-4.0 \mathrm{~mm}$ [1]. Rain is one of many weather effects that can exist on a given signal path. The difference between rain attenuation and other clear-sky effects is that rain is considered to be an "excess" attenuation in reference to the ideal weather 
situation. The excess attenuation that is endured by a propagating signal is comprised of two main components, absorption and scattering. The total excess attenuation in $\mathrm{dB}$ of a signal can be written mathematically as the sum of the absorption and scattering parameters as:

$$
A_{e x}=A_{a b}+A_{s c},
$$

where the subscripts represent excess attenuation, absorption attenuation, and scattering attenuation, respectively.

J.E. Allnutt stated that an electromagnetic wave is said to be scattered when "its energy is redirected from the original propagation direction without losing any substantial energy to the scattering particle, or particles" in [1]. This scatter can occur in any direction, as long as it is redirected from the original path.

Absorption and scattering are dependent on the complex index of refraction, which itself has dependencies on wavelength, temperature, and the interacting particle's size. When the particle is electrically small, Rayleigh theory can be applied to describe the radio wave interaction. The theory states that after a particle scatters a signal, the medium remains unchanged. This then leads to the particle appearing to be a small radiating dipole, which can be described by traditional electromagnetic theory. This approximation generally holds true for frequencies $10 \mathrm{GHz}$ and below when the interaction is dealing with hydrometeors.

When higher frequency analysis is required for interaction with hydrometeors, Rayleigh scattering theory can no longer be applied, since the interacting particle is no longer electrically small. As the wavelength decreases below the radius of a rain drop, the primary attenuation is derived from absorption rather than scattering. Furthermore, the imaginary component of the index of refraction becomes significant at frequencies above $10 \mathrm{GHz}$. These cases are best described using Mie scattering theory, which can be visually conveyed in Figure 4 [9]. 


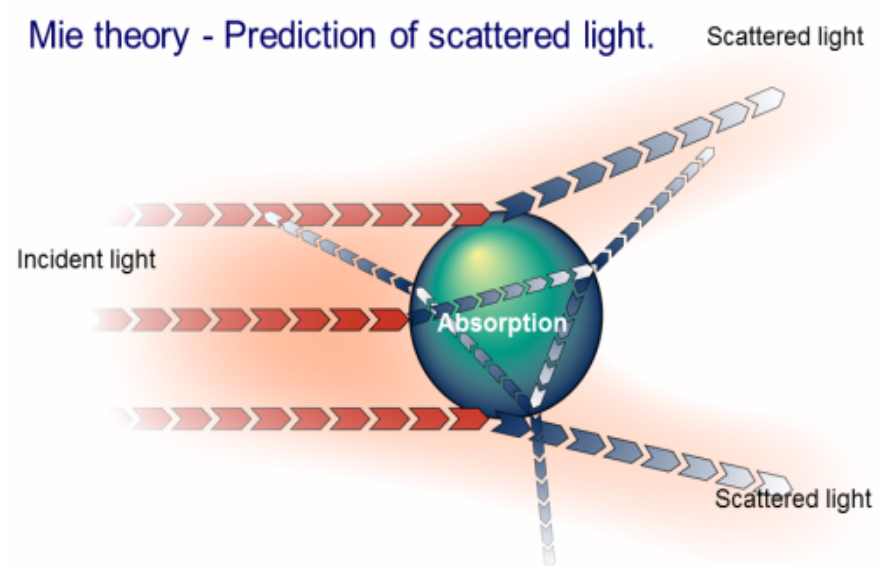

Figure 4. The Mie Solution Scattering Phenomenon

The Mie scattering theory describes the scattering of electromagnetic energy by a sphere. The formulation described in the theory allows for calculations of the electric and magnetic fields outside and within the particle of interest in order to determine how much energy is scattered. Mie theory defines an efficiency factor, provided effective extinction and scattering cross sections, which describes how a particle would interact with an electromagnetic wave. These relationships are:

$$
\begin{aligned}
& Q_{e x}=\frac{\sigma_{e x}}{\pi \times r^{2}}, \\
& Q_{s c}=\frac{\sigma_{s c}}{\pi \times r^{2}},
\end{aligned}
$$

where "ex" denotes the extinction factor, "sc" denotes the scattering factor, " $r$ " is the radius of the rain drop, and " $Q$ " represents the efficiency factor.

This relationship is very useful when examining a single interaction between a wave and a rain drop. However, it is often desired to understand the interaction of the radio wave and many rain drops. One possible way of calculating this is to integrate the extinction contributions with knowledge of the drop size distribution $N_{D}$, the path length $L$, and the extinction cross section of a rain drop with a specified drop diameter $C_{\mathrm{t}}(D)$. This can be seen as [1]:

$$
A_{e x}=4.343 \times L \int_{0}^{\infty} C_{t(D)} N_{(D)} d D \quad \mathrm{~dB} / \mathrm{km} .
$$

Evaluation of this integral is not simple and is typically not used in practical situations.

To make matters more complicated, the Mie solution to Maxwell's equations assumes a spherical 
radiator. Rain, unfortunately, is not always spherical. Rain drops tend to maintain a spherical shape when the diameter is approximately $170 \mu \mathrm{m}$ or less [1]. Above that, the aerodynamic forces change the drop shape to forms such as spheroidal, flattened spheroidal, and the Pruppacher and Pitter defined shape [1]. These different shapes also have a different impact on the wave known as depolarization. These shapes go against the Mie solution assumptions. Since rain characteristics vary vastly in time and space, empirical procedures have tended to be used to describe propagation through rain, often providing the best results.

The ITU-R 838-3 standard was developed in order to empirically determine the attenuation from 1-55 GHz with knowledge of rain rates (although they are not necessary). When rain rates are not provided, this standard utilizes statistical site data that have been collected for over 40 years in order to provide reasonable site data for modeling [9]. Furthermore, if the knowledge of rain rates are known, or measured, then attenuation due to specific rainfall rates for a given percentage of time can be calculated.

For the purposes of this work, the ITU-R standards P.618, P.837, P.838 and P.839 were referenced. ITU-R reference P.837 contains maps of meteorological parameters, which are used for rainfall rate statistics with a 1 minute integration time when local measurements are not available [9]. Furthermore, annex 1 of [9] describes the procedure necessary in order to derive rainfall rates from the provided parameters in the ITU-R database.

ITU-R P.838 standard calculates the attenuation due to rain from knowledge of rain rates. Since the characteristics of rain vary vastly in time and space, it is necessary to use statistical averaging or empirical methods in order to simplify the calculation [1]. This model came about from the understanding that the drop size distribution has a power-law relationship to rainfall rate. From this knowledge, coefficients were developed based on Marshall and Palmer, Laws and Parsons, and Joss drop size distributions [1]. Specifically, these coefficients were utilized for the calculation of specific attenuation. The specific attenuation due to rain, $\gamma R(\mathrm{~dB} / \mathrm{km})$, also follows a power-law relationship,

$$
\gamma_{R}=k R^{\alpha}
$$

where $R$ is the rain rate ( $\mathrm{mm} /$ hour), and $k$ and $\alpha$ are frequency dependent coefficients listed in the recommendation [2]. This is essentially the fundamental concept that the ITU-R P.838 recommendation adopted, which claims accuracy up to $1000 \mathrm{GHz}$. Determination of the specific 
attenuation, $\gamma_{\mathrm{R}}$, is highly relevant when there is a need to calculate attenuation with knowledge of rain rates.

In the specific attenuation relationship above, the frequency dependent coefficients can be calculated with the following formulas:

$$
\begin{gathered}
\log _{10} \mathrm{k}=\sum_{j=1}^{4} a_{j} \exp \left[-\left(\frac{\log _{10} f-b_{j}}{c_{j}}\right)^{2}\right]+m_{k} \log _{10} f+c_{k}, \\
\alpha=\sum_{j=1}^{5} a_{j} \exp \left[-\left(\frac{\log _{10} f-b_{j}}{c_{j}}\right)^{2}\right]+m_{\alpha} \log _{10} f+c_{\alpha},
\end{gathered}
$$

where $f$ is the frequency in $\mathrm{GHz}, k$ is either $k_{H}$ or $k_{V}$, and $\alpha$ is either $\alpha_{H}$ or $\alpha_{V}$. The subscripts, $H$ and $V$, are the variable's polarization designations where $H$ represents horizontal polarization and $V$ represents vertical polarization. The specific coefficients of $k$ and $\alpha$ can be calculated for linear and circular polarization as:

$$
\begin{gathered}
k=\left[k_{H}+k_{V}+\left(k_{H}-k_{V}\right)(\cos \theta)^{2} \cos 2 \tau\right] / 2, \\
\alpha=\left[\alpha_{H} k_{H}+\alpha_{V} k_{V}+\left(\alpha_{H} k_{H}-\alpha_{V} k_{V}\right)(\cos \theta)^{2} \cos 2 \tau\right] / 2 k,
\end{gathered}
$$

where $\theta$ is the elevation angle, and $\tau$ is the polarization tilt angle (for circular polarization, $\tau$ is $\left.45^{\circ}\right)$.

The ITU-R R.839 standard was intended to provide information regarding the height to which rain extends during periods of precipitation [10]. Basically, this model was intended to be used for areas of the world that no specific information is available. Additionally, this recommendation provides mildly accurate data for anywhere in the world when no site-specific information is present.

Finally, a section in the ITU-R P.618 standard ties all of this information into a model to estimate the long-term statistics for site-specific rain attenuation for frequencies up to approximately 55 GHz. The ITU-R 618-10 standard specifies the data required for typical EarthSpace telecommunications systems. Although this may seem out of scope, this model also lists information on tropospheric propagation. The limitation at $55 \mathrm{GHz}$ is troublesome and will be considered when advancing to higher frequencies. This ITU-R recommendation provides longterm statistics of slant-path rain attenuation endured by a propagating signal for a given location. The following step-by-step procedure is mentioned in [1]. In order to perform this estimation, the following parameters are required a priori: 
- $f$ - frequency $(\mathrm{GHz})$,

- $R_{0.01}-$ "point rainfall rate" for $.01 \%$ of an average year at a specified location ( $\mathrm{mm} / \mathrm{hour}$ ),

- $h s$ - the earth station's height above the mean sea level $(\mathrm{km})$,

- Note that this parameter can be obtained via ITU-R P.1511 when site specific data is unavailable.

- $\theta$ - elevation angle (degrees),

- $\varphi$ - latitude coordinate of the earth station (degrees), and

- $R_{e}$ - the effective radius of the Earth given as $8,500 \mathrm{~km}$.

Step 1: Determine the rain height, $h_{R}$, as given in Recommendation ITU-R P.839.

Step 2: For $\theta \geq 5^{\circ}$, compute the slant-path length, $L_{s}$, below the rain height from:

$$
L_{S}=\frac{\left(h_{R}-h_{S}\right)}{\sin \theta} \mathrm{km} .
$$

For $\theta<5^{\circ}$, the following formula can be used:

$$
L_{S}=\frac{2\left(h_{R}-h_{S}\right)}{\left((\sin \theta)^{2}+\frac{2\left(h_{R}-h_{S}\right.}{R_{e}}\right)^{1 / 2}+\sin \theta} \mathrm{km} .
$$

If $h_{R}-h_{s} \leq 0$, the predicted rain attenuation for any time percentage is zero and the following steps are not required.

Step 3: Calculate the horizontal projection, $L_{G}$, of the slant-path length from:

$$
L_{G}=L_{s} \cos \theta \mathrm{km} .
$$

Step 4: Obtain the rainfall rate, $R_{0.01}$, exceeded for $0.01 \%$ of an average year with an integration time of one minute. If this long-term statistic is unavailable from local data resources, an estimate can be obtained from the maps of rainfall rates given in ITU-R 837 [9]. If $R_{0.01}$ is equal to zero, the anticipated rain attenuation is also equal to zero for any percentage of time and the following steps are not required. 
Step 5: Obtain the specific attenuation, $\gamma \mathrm{R}$, using the frequency dependent coefficients given in [1] and ITU-R P.838, and the point rainfall rate, R0.01, determined in Step 4, by using:

$$
\gamma_{R}=k\left(R_{0.01}\right)^{\alpha} \mathrm{dB} / \mathrm{km} .
$$

Step 6: Calculate the horizontal reduction factor, $r_{0.01}$, for $0.01 \%$ of the time:

$$
r_{0.01}=\frac{1}{1+.78 \sqrt{\frac{L_{G} \gamma_{R}}{f}}-.38\left(1-e^{-2 L_{G}}\right)} .
$$

Step 7: Calculate the vertical adjustment factor, $v_{0.01}$, for $0.01 \%$ of the time:

$$
\zeta=\tan ^{-1}\left(\frac{h_{R}-h_{s}}{L_{G} r_{0.01}}\right) \text { degrees. }
$$

For $\zeta>\theta$,

$$
L_{R}=\frac{L_{G} r_{0.01}}{\cos \theta} \mathrm{km},
$$

else,

$$
L_{R}=\frac{\left(h_{R}-h_{S}\right)}{\sin \theta} \mathrm{km} .
$$

If $|\varphi|<36^{\circ}$,

$$
\chi=36-|\varphi| \text { degrees, }
$$

else,

$$
\chi=0 \text { degrees, }
$$

and

$$
\mathrm{v}_{0.01}=\frac{1}{1+\sqrt{\sin \theta}\left(31\left(1-\mathrm{e}^{-(\theta /(1+\chi))}\right) \frac{\sqrt{L_{R} \gamma_{R}}}{f^{2}}-45\right)} .
$$

Step 8: The effective path length:

$$
L_{E}=L_{R} \mathrm{v}_{0.01} \mathrm{~km} .
$$

Step 9: The predicted attenuation exceeded for $0.01 \%$ of an average year is obtained from:

$$
A_{0.01}=\gamma_{R} L_{E} \mathrm{~dB} .
$$


Step 10: The estimated attenuation to be exceeded for other percentages of an average year, in the range $0.001 \%$ to $5 \%$, is determined from the attenuation to be exceeded for $0.01 \%$ for an average year:

If $p \geq 1 \%$ or $|\varphi| \geq 36^{\circ}$ :

$$
\beta=0
$$

If $p<1 \%$ and $|\varphi|<36^{\circ}$ and $\theta \geq 25^{\circ}$ :

$$
\beta=-.005(|\varphi|-36)
$$

Otherwise:

$$
\begin{gathered}
\beta=-.005(|\varphi|-36)+1.8-4.25 \sin \theta \\
A_{p}=A_{0.01}\left(\frac{p}{0.01}\right)^{-\left(0.655+0.033 \ln (p)-0.045 \ln \left(A_{0.01}\right)-\beta(1-p) \sin \theta\right)} \mathrm{dB} .
\end{gathered}
$$

In summary, the above procedure accurately predicted long-term statistics of attenuation due to rain for specific locations. It is noted that year-to-year variability should be taken into account when comparing predictive models and measured data.

\subsubsection{Clouds and Fog}

In fixed satellite systems, signal attenuations tend to occur when traveling through clouds and/or fog. Below $10 \mathrm{GHz}$, the attenuation attributed by clouds and fog is nearly negligible [1]. Above $10 \mathrm{GHz}$, the attenuation due to clouds and fog increases substantially as frequency increases. Signal attenuation due to fog and clouds are due primarily to the scattering and absorption of the signal.

The primary difference between the cases is that water droplet particulates that occur in clouds and fog are considered to be much smaller in size than that of a hydrometeor (rain drop). The droplets in clouds and fog are assumed to be less than $0.01 \mathrm{~cm}$ in diameter [11], which holds a spherical shape as seen in [1]. Provided that the rain drop is approximately $100 \mu \mathrm{m}$ in diameter or less, the Rayleigh approximation can be used to understand the electromagnetic attenuation phenomenon within clouds and fog. This approximation is valid for frequencies below $200 \mathrm{GHz}$, with the limiting factor being the decreased wavelength [11]. For completeness, the underlying 
theory states that after a particle scatters a signal, the medium remains unchanged. The particle can then be approximated as a small radiating dipole for electromagnetic analysis.

ITU-R P.840-5 is intended to provide guidance for engineers to design satellite-to-ground telecommunications systems above $10 \mathrm{GHz}$, while accounting for cloud and fog attenuation [11]. The recommendation provides calculation of specific attenuation within clouds and fog, and provides a total-attenuation prediction method [11]. While it is not explicitly stated in the literature, the specific attenuation, $\gamma_{c}$, can be used to provide accurate attenuation estimations when the distance through the cloud and the liquid water content of the cloud is known. Unlike the rain model, which considers reflection, refraction, and absorption due to larger droplet sizes, the ITU-R P.840-5 assumes small droplets and only considers scattering. The specific attenuation within a cloud or fog is calculated as [11]:

$$
\gamma_{c}=K_{l} M \mathrm{~dB} / \mathrm{km}
$$

where $K_{l}$ is the specific attenuation coefficient, and $M$ is the liquid water density in the fog $\left(\mathrm{g} / \mathrm{m}^{3}\right)$. This calculation of this coefficient utilizes a double-Debye model for the dielectric constant, which is the basis of Rayleigh scattering model [11]. The specific attenuation constant can be calculated accurately up to $1 \mathrm{THz}$ by:

$$
K_{l}=\frac{0.819 f}{\varepsilon^{\prime \prime}\left(1+\eta^{2}\right)}(\mathrm{dB} / \mathrm{km}) /\left(\mathrm{g} / \mathrm{m}^{3}\right)
$$

where $f$ is the frequency $(\mathrm{GHz})$, and $\eta$ is defined as:

$$
\eta=\frac{2+\varepsilon^{\prime}}{\varepsilon^{\prime \prime}}
$$

The parameters $\varepsilon$ ' and $\varepsilon$ " represent the real and imaginary parts of the dielectric permittivity of water, respectively. The dielectric permittivity is then given by:

$$
\begin{aligned}
& \varepsilon^{\prime \prime}(f)=\frac{f\left(\varepsilon_{0}-\varepsilon_{1}\right)}{f_{p}\left[1+\left(f / f_{p}\right)^{2}\right]}+\frac{f\left(\varepsilon_{1}-\varepsilon_{2}\right)}{f_{s}\left[1+\left(f / f_{s}\right)^{2}\right]}, \\
& \varepsilon^{\prime}(f)=\frac{\varepsilon_{0}-\varepsilon_{1}}{\left[1+\left(f / f_{p}\right)^{2}\right]}+\frac{\varepsilon_{1}-\varepsilon_{2}}{\left[1+\left(f / f_{s}\right)^{2}\right]}+\varepsilon_{2},
\end{aligned}
$$

where

$$
\begin{gathered}
f_{p}=20.09-142(\theta-1)+294(\theta-1)^{2} \mathrm{GHz} \\
f_{s}=590-1500(\theta-1) \mathrm{GHz} \\
\varepsilon_{0}=77.6+103.3(\theta-1) \\
\text { Approved for Public Release; Distribution is Unlimited.. }
\end{gathered}
$$




$$
\begin{aligned}
\varepsilon_{1} & =5.48, \\
\varepsilon_{2} & =3.51, \\
\theta & =\frac{300}{T},
\end{aligned}
$$

with the temperature in Kelvin. In the above equations, the variables $f_{p}$ and $f_{s}$ are defined as the principal and secondary relaxation frequencies [11]. From this point, total attenuation due to clouds or fog can be calculated with a linear calculation of the specific attenuation parameter and the propagation distance through the medium.

The attenuation can also be estimated using known site-specific statistics of total columnar content of liquid water, or millimeters of water that is able to precipitate. The attenuation is then estimated by:

$$
A=\frac{L K_{l}}{\sin \theta} 90^{\circ} \geq \theta \geq 5^{\circ},
$$

where $L$ is the liquid water content, $K_{l}$ is the specific attenuation coefficient, and $\theta$ is the elevation angle. The statistics mentioned above are provided in the ITU-R database.

In summary, this model utilizes the Rayleigh scattering approximation for small particles. This model provides the flexibility of only requiring a frequency and channel length, or the frequency, site location, and elevation angle, which are all generally known parameters. Another prediction method discussed in [1] takes a different approach in that it estimates attenuation with knowledge of the interfering cloud type. From this, it provides an estimation of the spatial volume that the cloud takes up, and more accurately, determines the distance the signal propagates through the medium [1].

\subsubsection{Dust and Particulates}

Electromagnetic attenuation resulting from interaction with dust particulates is a relatively under-modeled occurrence. However, there are groups that are devoting their efforts to characterizing the interaction [12]. When a radio wave interacts with a dust particle, the underlying mathematical theory follows Rayleigh (and Mie at higher frequencies) scattering theory as discussed in the preceding sections. Unlike water particles, dust particles vary greatly in size and electrical characteristics, since the composition of dust is not comprised of only one molecule. 
The power loss endured by the incident wave can be calculated by multiplying the uniform power density with the cross-section of the attenuator of interest [12]. The total power removed from the incident field through excess attenuation can be represented by an extinction cross-section $[12,13]$. As in the rain attenuation section, the attenuation due to dust storms can be calculated similarly as [12]:

$$
A_{T}=\int_{0}^{L} A_{p} d x \mathrm{~dB}
$$

with the specific attenuation, $A_{p}$, given as

$$
A_{d}=4.343 \times 10^{3} \int_{a_{\min }}^{a_{\max }} \sigma_{t}(a) N(a) d a \mathrm{~dB} / \mathrm{km},
$$

where $\sigma_{t}$ is the total attenuation cross-section factors of particles with radius $a$, and $N(a)$ is defined as the number or particles per unit volume of air with particles of radius of $a_{\max }-a_{\min }$. As in the case of rain prediction methods, obtaining an accurate distribution of particles is no simple task. Therefore, Rayleigh and Mie approximations have been made to describe the interaction.

The Rayleigh approximation for the specific attenuation due to dust storms is given in $[12,14]$ by Islam and Goldhirsh respectively as:

$$
A=\frac{2.317 \times 10^{-3} \cdot \varepsilon^{\prime \prime}}{\left[\left(\varepsilon^{\prime}+2\right)^{2}+\varepsilon^{\prime 2}\right] \cdot \lambda} \cdot \frac{1}{V^{\gamma}} \mathrm{dB} / \mathrm{km},
$$

where $V$ is the visibility in kilometers, $\lambda$ is the wavelength in meters, $\gamma$ is the locational and temporal parameter, and $\varepsilon$ ' and $\varepsilon$ " are the real and imaginary parts of the dielectric constant of the dust particles in question [12]. This formulation developed by Goldhirsh claims accuracy up to $48 \mathrm{GHz}$ [14].

As with hydrometeors, the Rayleigh approximation cannot be assumed when the wavelength becomes comparable to the size of the dust particle. Therefore, the Mie approximation for dust storms is generally used to describe attenuation at higher frequencies. The specific attenuation for dust storms is given in [12] as:

$$
A_{d}=4.343 \times 10^{3} \int_{a_{\min }}^{a_{\max }}\left[\frac{\lambda^{2}}{2 \pi}(k a)^{3}\left(c_{1}+c_{2}(k a)^{2}+c_{3}(k a)^{3}\right)\right] N(a) d a \mathrm{~dB} / \mathrm{km},
$$

where the bracketed terms represent total cross-section efficiency as mentioned previously for dust storm attenuation.

The author extends these models to a custom dust storm model, which provides a general formula for the frequency range 1-100 GHz. The general formulation from [12] is as follows: 


$$
A_{d}=\frac{a_{e} f}{V}\left[x+y a_{e}^{2} f^{2}+z a_{e}^{3} f^{3}\right] \mathrm{dB} / \mathrm{km}
$$

where $a_{e}$ is the equivalent particle radius in meters (which was approximated to $50 \mu \mathrm{m}$ in [12]), $f$ is the frequency in $\mathrm{GHz}, V$ is the visibility in kilometers, and $x, y$, and $z$ are constants whose values are dependent on the complex dielectric constant [12]. These values are given as:

$$
\begin{gathered}
x=\frac{1886 \cdot \varepsilon \prime \prime}{\left(\varepsilon^{\prime}+2\right)^{2}+\varepsilon^{\prime \prime}{ }^{2}}, \\
y=137 \times 10^{3} \cdot \varepsilon^{\prime \prime}\left\{\frac{6}{5} \frac{7 \varepsilon^{\prime 2}+7 \varepsilon^{\prime \prime}+4 \varepsilon^{\prime}-20}{\left[\left(\varepsilon^{\prime}+2\right)^{2}+\varepsilon^{\prime \prime}\right]^{2}}+\frac{1}{15}+\frac{5}{3\left[\left(2 \varepsilon^{\prime}+3\right)^{2}+4 \varepsilon \prime^{\prime 2}\right]}\right\},
\end{gathered}
$$

and

$$
z=379 \times 10^{4}\left\{\frac{\left(\varepsilon^{\prime}-1\right)^{2}\left(\varepsilon^{\prime}+2\right)+\left[2\left(\varepsilon^{\prime}-1\right)^{2}\left(\varepsilon^{\prime}+2\right)-9\right]+\varepsilon^{\prime \prime}}{\left[\left(\varepsilon^{\prime}+2\right)^{2}+\varepsilon^{\prime \prime}\right]^{2}}\right\} .
$$

The model was further simplified in [12] to equations that were frequency band specific. These simplified relationships were achieved by determining approximate complex dielectric constants for frequency bands. Specifically, the author of [12] utilized measured values to make these approximations.

Ultimately, this model is one of the few that describes an attenuation prediction method for propagation within dust storms. The ITU-R references the issue and states that "very little is known about the effects of sand and dust storms on radio signals on slant-paths". That being said, this model is not widely held as the standard of the world, although none are. The author described both a prediction method and data validation in [12], which gives the presented model some validity.

\subsubsection{Scintillation}

When the atmosphere is still, the refractive index varies slowly with height, and more slowly in the horizontal plane [1]. The presence of wind causes the atmosphere to be mixed, and results in small-scale rapid variations in the refractive index [1]. These rapid variations are referred to as tropospheric scintillations. "The magnitude of tropospheric scintillations depends on the magnitude and structure of the refractive index variations, increasing with frequency and with the path length through the medium, and decreasing as the antenna beamwidth decreases because of aperture averaging". For systems operating at low frequencies or having large-fade margins, scintillation effects do not severely impact the communications system $[1,15]$. In satellite communications, this is often not the case.

Approved for Public Release; Distribution is Unlimited. 
These fluctuations in the index of refraction along a propagation path result in amplitude and phase scintillations in the signal, and ultimately cause the received signal to have amplitude and phase deviations [1]. Tropospheric scintillation is said to be comprised of two components. The first component is due to energy dispersive small scale variations in the refractive index of the lower troposphere $[1,16]$.The second component is due to the turbulent mixing at the edges of clouds [1]. This component tends to the more intense than those in free-space.

Tropospheric scintillation has been characterized to have dependence on meteorological, temporal, geographic, systematic, and frequency variance. These dependencies are important to consider when investigating attenuation effects due to tropospheric scintillations.

Studies have proven that tropospheric scintillation is strongly correlated with temperature and humidity [1]. When both parameters are high, the scintillation effect on signal amplitude also increases. Evidence has also shown that increasing wind speeds result in slightly increasing amplitude scintillations [1]. One attenuation effect of interest is the scintillation correlation with rainfall. Many studies have been conducted to determine this, and have reported largely increased amplitude scintillation effects [16]. Another area of interest is the impact that increasing the frequency has on tropospheric scintillations. When the same antenna is utilized to measure scintillations at different frequencies, high correlation exists.

The ITU-R P.618-10 recommendation specifies a method in section 2.4 to estimate attenuation due to amplitude scintillation for elevation angles greater than $4^{\circ}$. This model requires known values for the frequency, $f$, site average ambient surface temperature, $t$, humidity, $H$, the elevation path angle, $\theta$, the physical diameter (or largest dimension) of the antenna, $D$, the antenna efficiency, $\eta$, and the "wet term" of the index of refraction. The "wet term" is determined by ITU-R recommendation P.453. This procedure is as follows:

Step 1: For the values of $t$, calculate the saturation water vapor pressure, $e_{s},(\mathrm{hPa})$, as specified in Recommendation ITU-R P.453.

Step 2: Compute the wet term of the refractive index, $N_{\text {wet }}$, corresponding to $e_{s}, t$ and $H$ as given in Recommendation ITU-R P.453. Note that steps 1 and 2 are not needed if $N_{\text {wet }}$ is obtained from Recommendation ITU-R P.453 directly.

Step 3: Calculate the standard deviation of the signal amplitude, $\sigma_{r e f}$, used as reference:

$$
\sigma_{\text {ref }}=3.6 \times 10^{-3}+10^{-4} \times N_{w e t} \mathrm{~dB} .
$$


Step 4: Calculate the effective path length $L$ according to:

$$
L=\frac{2 h_{L}}{\sqrt{\sin ^{2} \theta+2.35 \times 10^{-4}}+\sin \theta} \mathrm{m},
$$

where $h_{L}$ is the height of the turbulent layer $\left(h_{L}=1000 \mathrm{~m}\right)$.

Step 5: Estimate the effective diameter, $D_{\text {eff }}$, from the geometrical diameter, $D$, and the antenna efficiency $\eta$ :

$$
D_{e f f}=\sqrt{\eta} D \mathrm{~m}
$$

Step 6: Calculate the antenna averaging factor:

$$
g(x)=\sqrt{3.86\left(x^{2}+1\right)^{11 / 12} \cdot \sin \left[\frac{11}{6} \tan ^{-1} \frac{1}{x}\right]-7.08 x^{5 / 6}}
$$

with:

$$
x=1.22 D_{\text {eff }}^{2}(f / L),
$$

and $f$ is the operation frequency. If the argument of the square root is negative when $\mathrm{x} \geq 7$, the predicted scintillation fade depth for any percentage of time is zero and the remaining procedure is not required.

Step 7: Calculate the standard deviation of the signal for the considered period and propagation path:

$$
\sigma=\sigma_{r e f} f^{7 / 12} \frac{g(x)}{(\sin \theta)^{1.2}}
$$

Step 8: Calculate the time percentage factor, $a(p)$, for the time percentage, $p$, of concern in the range $.01<p \leq 50$ :

$$
a(p)=-0.061\left(\log _{10} p\right)^{3}+0.072\left(\log _{10} p\right)^{2}-1.71 \log _{10} p+3.0 .
$$

Step 9: Calculate the scintillation fade depth for the time percentage $p$ by:

$$
A_{s}(p)=a(p) \cdot \sigma \mathrm{dB}
$$

Unfortunately, this model is only tested up to $20 \mathrm{GHz}$ and is recommended for use up to $30 \mathrm{GHz}$. This is the major limitation of the link-budget GUI, since higher frequencies can experience higher losses due to tropospheric turbulences. Though, it is generally a good practice to account for known losses, rather than ignoring them completely. 


\subsubsection{Free-Space Path Loss}

Consider a signal that is transmitted through free-space to a receiver located at distance $d$ from the transmitting system. Also assume that no excess attenuation or obstacles are present, and the signal propagates along a clear signal path directly between the transmitter and receiver. This channel type is known as a line-of-sight (LOS) channel. The losses endured by the signal simply propagating in this situation are known as path loss or free-space path loss in this case [17].

The free-space path loss is the decrease in power that the signal experiences as it travels the distance $d$ to the receiver. The loss experienced will be proportional to the inverse of the square of the distance $\left(1 / d^{2}\right)$ [1]. The power flux density for an isotropic antenna transmitting with a power $P_{t}$ at distance $d$ can be given by:

$$
p_{f d}=\frac{P_{t}}{4 \times \pi \times d^{2}} \mathrm{~W} / \mathrm{m}^{2} .
$$

Note that the denominator term is simply the radius of a sphere of radius $d$. If the antenna considered is no longer isotropic, but rather has a gain associated with it called $G_{t}$, then the power flux density becomes [4]:

$$
p_{f d}=\frac{P_{t} \times G_{t}}{4 \times \pi \times d^{2}} \mathrm{~W} / \mathrm{m}^{2} .
$$

Multiplying the power flux density by the receiving antenna aperture area, results in the received power. Since the gain of an antenna can be related to its area, the receiver gain can be extracted from the previous product. This results in the scalar quantity:

$$
P_{r}=P_{t} \times G_{t} \times\left(\frac{\lambda}{4 \times \pi \times d}\right)^{2} \mathrm{~W} .
$$

The term within the parenthesis can be inverted, and would be referred to as the free-space path loss. This equation has been used throughout the years and has been found to be very useful in developing link-budgets.

The free-space path loss model is not very different from the equations given above. The only difference is that in the model used, the free-space path loss attenuation is converted to $\mathrm{dB}$ losses. This is given as:

$$
F S P L(d B)=20 \log _{10}(d)+20 \log _{10}(f)+20 \log _{10}\left(\frac{4 \pi}{c}\right) d B,
$$


where $d$ is the distance in meters, $f$ is the frequency in $\mathrm{Hz}$, and $c$ is the speed of light in a vacuum. This is a fundamental loss factor that is taken into account every time a link-budget is performed.

\subsubsection{Total Attenuation Due to Multiple Sources}

When multiple attenuation effects are present and occurring simultaneously, it is important to understand how the net loss is calculated from the presented attenuation models. The net loss estimated is not a simple addition of each calculated parameter. This is because some of the model presented overlap in the losses that they estimate. Specifically, when rain is present, the models of gaseous absorption and clouds and fog attenuation must be calculated for differently, since the rain model accounts for some losses that are normally determined in their models.

After values for the attenuation due to rain, clouds, gasses, and tropospheric scintillation are calculated, they can be combined using the following model:

$$
A_{T}(p)=A_{G}(p)+\sqrt{\left(A_{R}(p)+A_{C}(p)\right)^{2}+A_{S}^{2}(p)},
$$

where $A_{G}$ is the attenuation due to gasses, $A_{R}$ is the attenuation due to rain, $A_{C}$ is the attenuation due to clouds and/or fog, and $A_{S}$ is the attenuation due to tropospheric scintillation all for a given probability of time of the attenuation [17].

When $A_{R}$ is non-zero, special considerations must be made for the gaseous and clouds and fog attenuation models. When the probability is less than $1 \%$, then the contributions from the gaseous absorption and clouds and fog models must be calculated at a probability of $1 \%$.

Mathematically, this is seen as:

$$
\begin{aligned}
& A_{C}(p)=A_{C}(1 \%) \text { for } \mathrm{p}<1 \%, \\
& A_{G}(p)=A_{G}(1 \%) \text { for } \mathrm{p}<1 \% .
\end{aligned}
$$

As stated above, these equations take into account the fact that a large part of cloud and fog, and gaseous attenuation is already included in the rain attenuation prediction model for percentage of times below $1 \%$.

Approved for Public Release; Distribution is Unlimited. 


\subsubsection{Coupled Model}

The attenuation models discussed previously were translated into MATLAB, and a coupled model was created in the form of a graphical user interface (GUI). The development of a GUI allows for timely and precise link-budget calculations and loss estimations from which transmitting and receiving systems can be specified. The development of this model was an essential step towards creating a baseline model to be used and extrapolated at higher frequencies, especially considering the atmospheric impairments for EHF.

The link-budget GUI shown in Figure 5 allows for a multitude of different test scenarios. It models the standard atmospheric effects based on world location (for highest accuracy), and can include attenuation effects such as rain, clouds, fog, dust storms, and others as needed. This degree of flexibility allows for specification and planning for "worst-case" scenarios to ensure optimum performance.

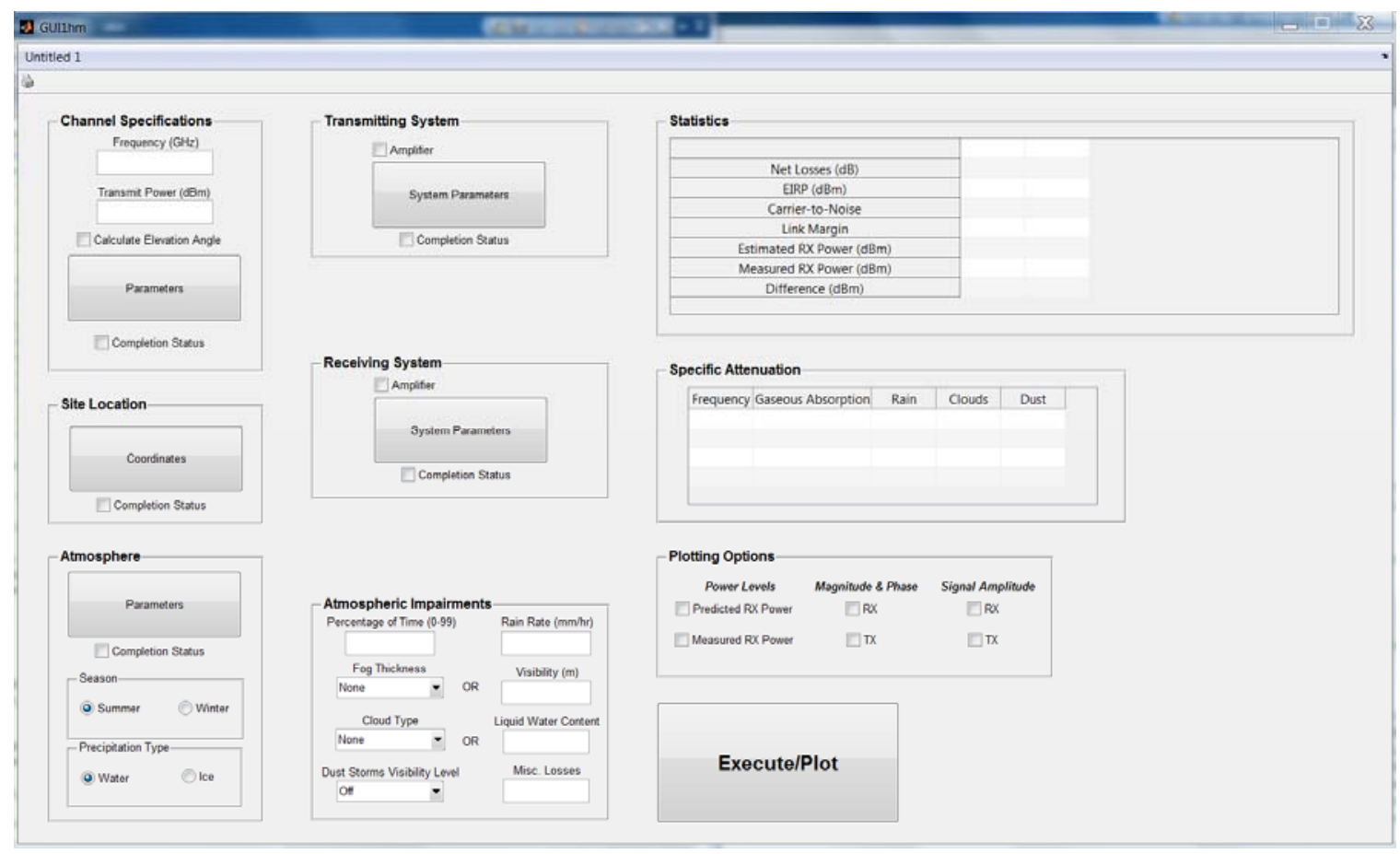

Figure 5. The Link-Budget GUI

The GUI allows for the designer/user to test numerous scenarios which can ultimately result in maximum accuracy when determining the system specifications. To achieve precision in 
the results, one must go through the "fine-tuning" of the link budget to ensure the minimum amount of power is received, while not wasting resources with "over-kill” designs. The following sections will discuss the GUI in detail.

\subsubsection{Channel Specifications}

This portion of the GUI allows the designer to specify the fundamental parameters for the channel. For example, if the designer knows that he/she wants to use $80 \mathrm{GHz}$, and has a transmitter that supports that, then it can be set. Additionally, if the transmitter has a low transmit power without an amplifier, then setting the maximum power output of the transmitter will assist with the development of the entire system.

The parameters in this section that can be changed are the frequency, transmission power, channel length, receiver elevation, transmitter elevation, and optionally the elevation angle. For most Earth-space communication systems, the elevation angle is a set parameter. This is not the case though if on-ground experimentation is desired. Due to the close proximity of the transmitter and receiver in this case, the option for the program to calculate the elevation angle is beneficial. The channel specification panel and associated "pop-up" menu can be seen in Figure 6.
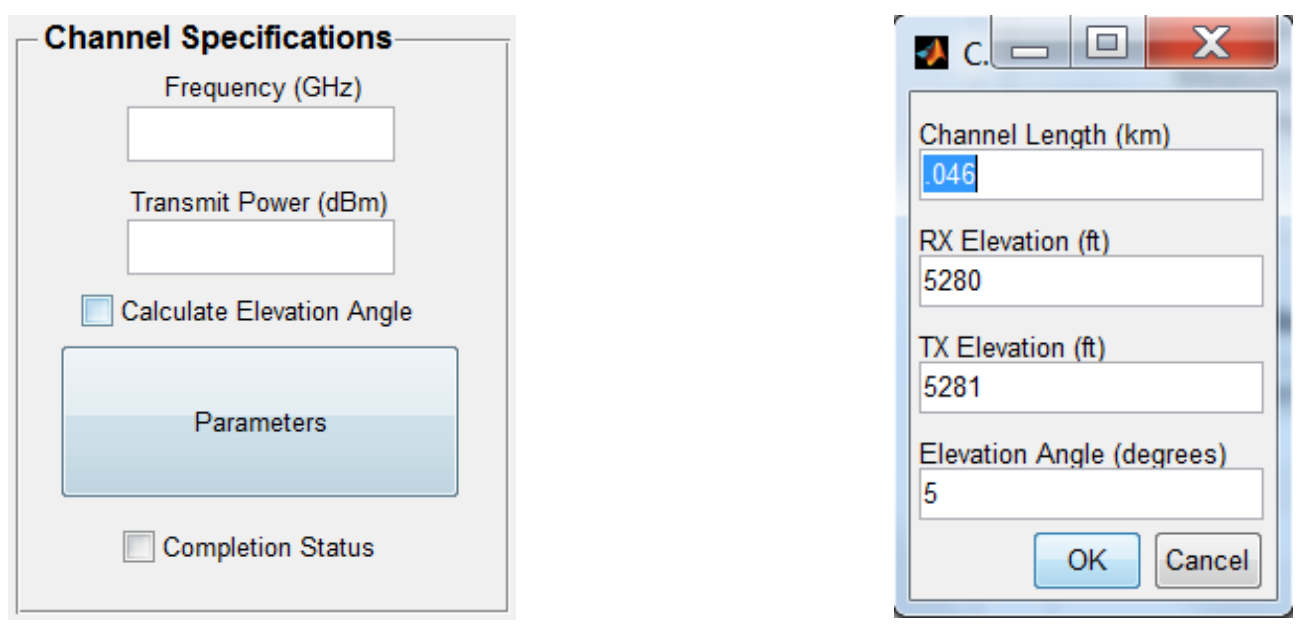

Figure 6. (a) The Channel Specification Panel (Left) Is What The User Normally Sees On The Main GUI; (b) The Pop-up Menu (Right) Is What The User Sees When The "Parameters" Button Has Been Selected 
In addition, each parameter has limits that are specified and enforced in the program. The frequency entry can be an array of single valued elements. This is advantageous for results requiring frequency sweeps.

$$
1 \leq \text { frequency } \leq 100 \mathrm{GHz} \text {. }
$$

The "Transmit Power" entry is the user set power without the addition of any losses. The entry must be a real power, but has no value limitations:

$$
\mathbb{R}\left\{P_{T}\right\}=P_{T} .
$$

The "Channel Length" must be greater than $0 \mathrm{~km}$, but can be a very small fraction if desired. The upper limit is set at $100 \mathrm{~km}$, although, at large distances, some computations can become intense and time consuming. The upper limit can be removed if the processing unit is capable:

$$
0<C L \leq 100 \mathrm{~km} \text {. }
$$

The elevation angle has the range of:

$$
0<\theta \leq 90 \text { degrees. }
$$

The calculation of the "Elevation Angle", if selected, is performed by simple trigonometry. The angle is determined by:

$$
\theta=\sin ^{-1} \frac{\left|h_{T X}-h_{R X}\right|}{C L},
$$

where $C L$ is the channel length, $h_{T X}$ is the height of the transmitter, and $h_{R X}$ is the height of the receiver.

\subsubsection{Site Location}

The site location is a straight-forward, yet highly important parameter. If the region is known in which the communication system will be used, then statistical data on rain rates and other atmospheric impairments can be utilized in the system design. Ultimately, this section requires the user to enter both latitude and longitude values. The panel and pop-up menu can be seen in Figure 7. 

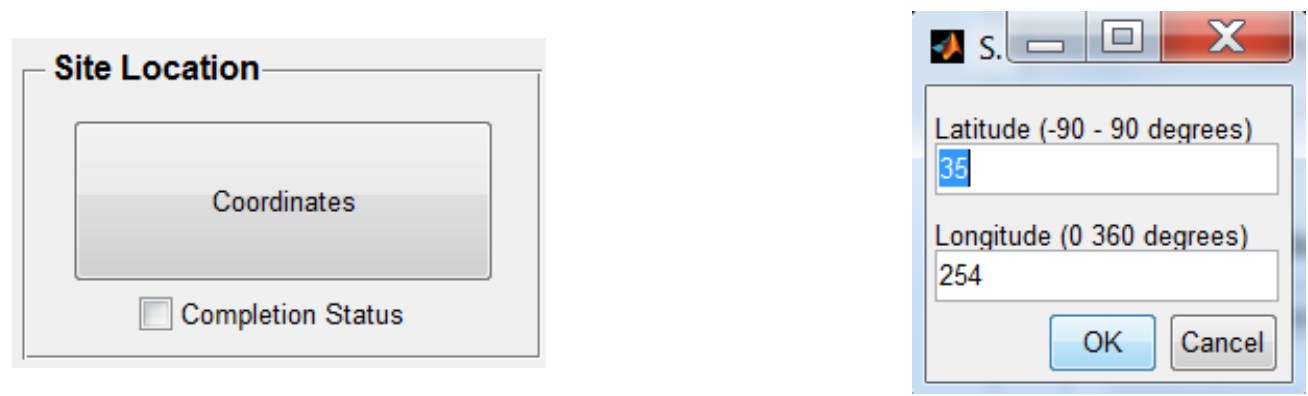

Figure 7. (a) The Site Location Panel (Left) Is What The User Normally Sees On The Main GUI; (b) The Pop-up Menu (Right) Is What The User Sees When The “Coordinates" Button Has Been Selected

\subsubsection{Atmosphere}

The entries on this panel require specific atmospheric parameters (which are usually readily available) or long-term atmospheric data in order to determine the system limitations from an atmospheric perspective. This panel allows for the user to enter temperature, air pressure, humidity, season, and precipitation type. The first four parameters seem straight forward, but the precipitation type is a little arbitrary. This selection allows the user to specify if the signal interacts with ice or water in the troposphere. This parameter is slightly arbitrary, and could generally be chosen with the associative season. This is seen in Figure 8.
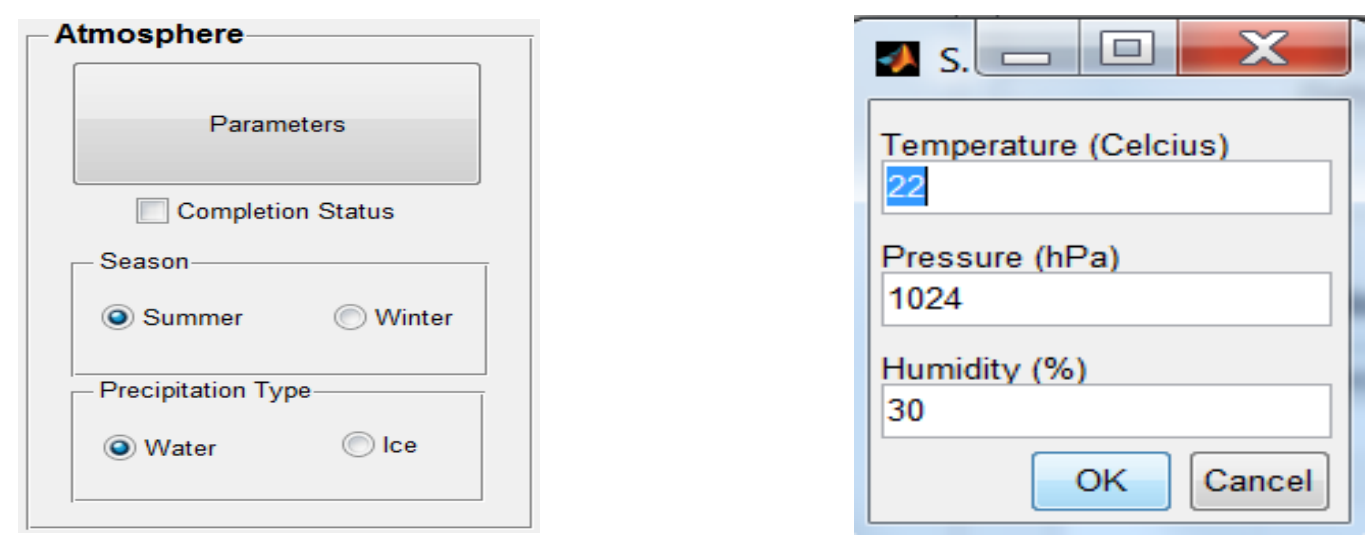

Figure 8. (a) The Atmosphere Panel (Left) Is What The User Normally Sees On The Main GUI; (b) The Pop-up Menu (Right) Is What The User Sees When The "Parameters" Button Has Been Selected 
The parameters ranges are as follows:

$$
\begin{gathered}
-273.15^{\circ} \mathrm{C} \leq \text { Temperature, } \\
0 \leq \text { Pressure } \leq 1085.7 \mathrm{hPa}, \\
0 \leq \text { Humidity } \leq 100 \%,
\end{gathered}
$$

The selection of seasons is left up to the best judgment of the user, also depending on the site location. For example, if the site were a location in the tropical region of the world, then a good rule of thumb would be to select summer. Making selections in the mid-latitudes could make the decisions more difficult. The precipitation type can be determined by the user's judgment. Knowing the practical site environment could make this decision easier, as well as the time of year.

\subsubsection{Transmitting and Receiving System}

In the GUI, the transmitting and receiving systems essentially have the same input parameters. Both panels call for the antenna gain, antenna diameter or largest dimension, antenna efficiency, system cable losses, and optionally amplifier gain and saturation power. Although both panels are similar, it is rare for both the transmitter and receiver to have the same system elements. The panels and associated pop-up menus can be seen in Figure 9 and Figure 10. An example of each pop-up is conveyed through these images.
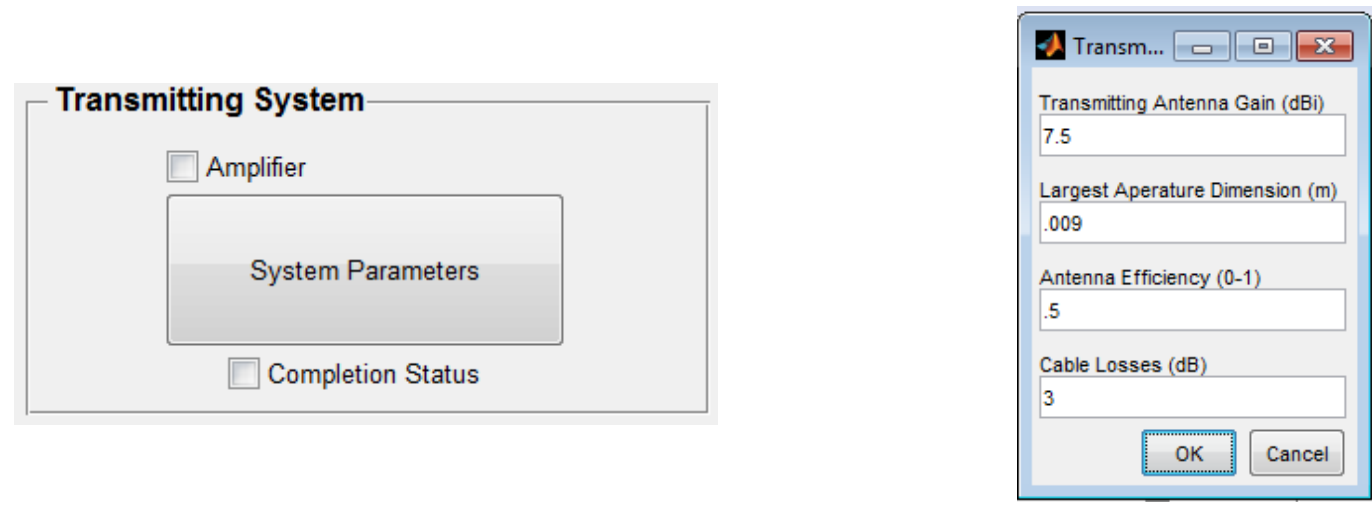

Figure 9. (a) The Transmitting Panel (Left) Is What The User Normally Sees On The Main GUI; (b) The Pop-up Menu (Right) Is What The User Sees When The "System Parameters” Button Has Been Selected; Note That The Amplifier Button Is Not Selected In This Case 
- Transmitting System

$\square$ Amplifier

System Parameters

Completion Status

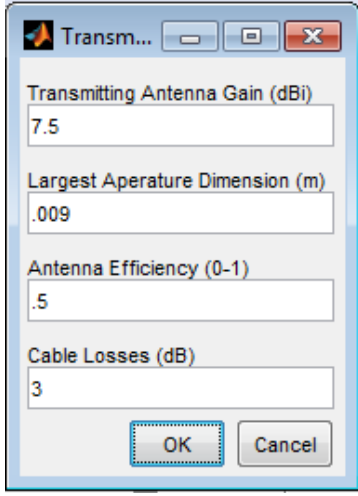

Figure 10. (a) The Receiving Panel (Left) Is What The User Normally Sees On The Main GUI; (b) The Pop-up Menu (Right) Is What The User Sees When The "System Parameters” Button Has Been Selected; Note That The Amplifier Button Was Selected In This Case

\subsubsection{Atmospheric Impairments}

The "Atmospheric Impairments" panel as seen in Figure 11, allows for the user to plan for a "worst-case" scenario in which rain, dust, fog, and clouds can be selected with a certain severity. The drop-down menus are intended for the general case, or when not much site information is provided. Each of the parameter sets and drop-down menus will be discussed in the following paragraphs.

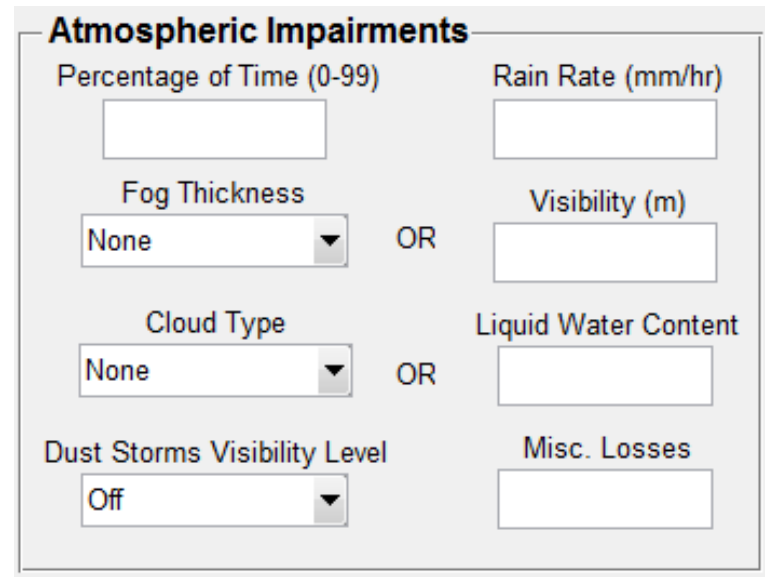

Figure 11. Excess Attenuation Panel 
The "Percentage of Time" is an input that takes in the percentage of time that attenuation exceeds a certain limit, usually for the worst month. For example, if a system operates in a desert-like climate with low precipitation, then a percentage of time that attenuation for the worst month would be not be exceeded due to rain could be $95 \%$. However, if the location were in a tropical rain forest, then then threshold might not be exceeded for only $35 \%$ of the time. Note that when the probability of time is chosen to exceed the threshold only $0.01 \%$ for example, intense attenuation can be expected.

The rain rate entry allows for the designer to add rain attenuation onto the signal. If the designer has a rain rate in mind, or is trying to find a system limitation, then specific values can be entered. If nothing is entered, then the program references yearly statistical rain data for the location entered. Ultimately, it provides the rainfall rate exceeded for a percentage of time during an average year.

The "Dust Storms" drop-down menu as seen in Figure 12 is a feature that allows the designer to take into account the signal attenuation that may occur during propagation through intense dust. The intensity of the storms is classified by the visibility levels, where the most intense is the lowest visibility level. It utilizes certain visibility levels where the smallest distance is the most severe case.

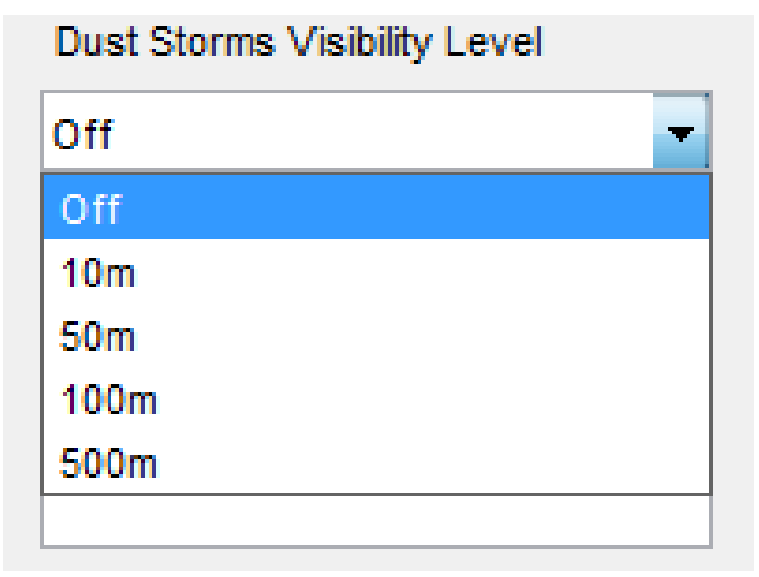

Figure 12. Dust Storms Visibility Level Drop-Down Menu; Note That The Lower The Visibility, The More Severe The Attenuation Is Expected To Be

Although the cloud and fog attenuation contributions are estimated using the same models, the perception of both is very different to the user. Therefore the parameters were 
seperated for operator convenience. The fog section also allows for the designer to either select the fog density or the visibility level. The "Fog Thickness" drop-down allows for the selection of different levels of density and is meant for the general case. The visibility parameter entry calculates the anticipated fog water content based on the visibility level. This is the better choice when more accuracy is required.

Finally, the cloud section allows the designer to anticipate the impact of clouds on the link. The designer has the choice of selecting cloud type, or entering the liquid water content in $\mathrm{g} / \mathrm{m}^{3}$. This section differs from that of the fog section in that clouds typically have a much higher water content that fog does.

\subsubsection{Plotting Options}

This section of the GUI is straight forward as seen in Figure 13. The designer has the option to plot predicted received power, signal amplitude, and the expected magnitude and phase for the transmitting and receiving systems. Note that there is a selection that states "Measured RX Power". This portion is intended to compare the predictive model results to measured results and requires the user to save data to an excel spreadsheet, to ensure the number of predicted power elements match the number of measured power elements.

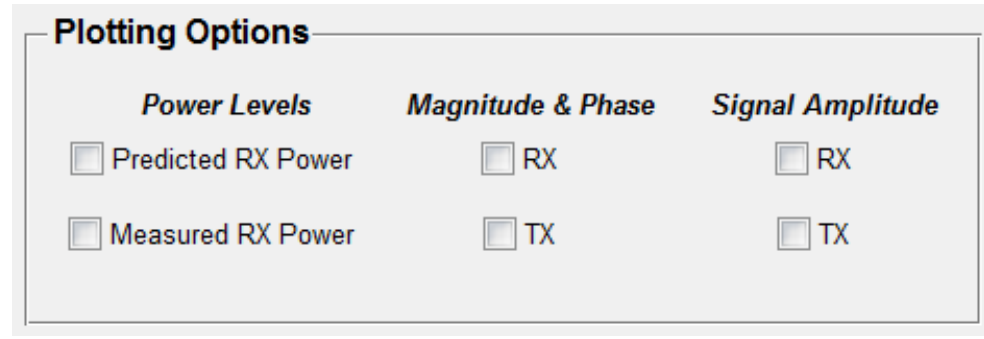

\section{Figure 13. The Plotting Options Panel}

\subsubsection{GUI Outputs}

From all of the user inputs, the coupled model determines the specific attenuation due to gaseous absorption, rain, clouds, and dust (Figure 14). The program automatically fills out the 
"Statistics" table, which lists the net losses, Effective Isotropic Radiated Power (EIRP), carrierto-noise, link margin, estimated received power, and phase parameters (Figure 15).

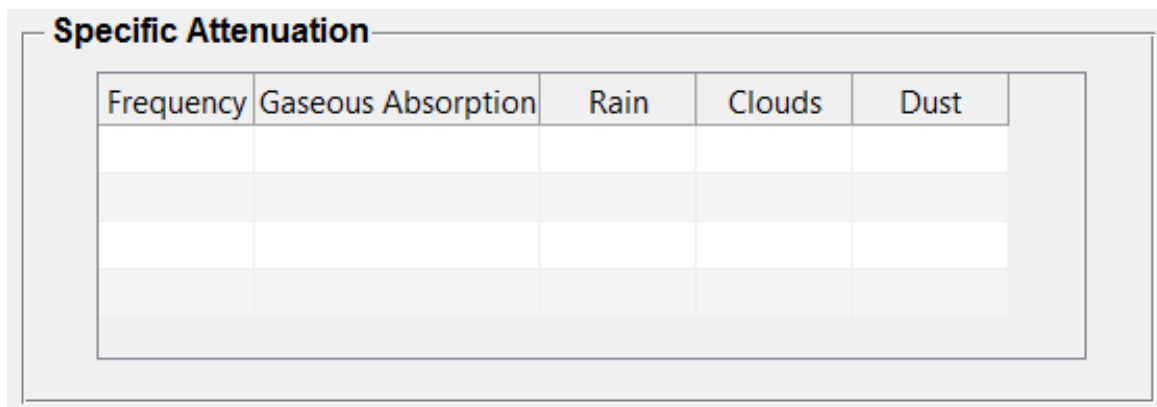

Figure 14. Specific Attenuation Panel Which Only Lists The Active Attenuators In The Link-Budget Calculation

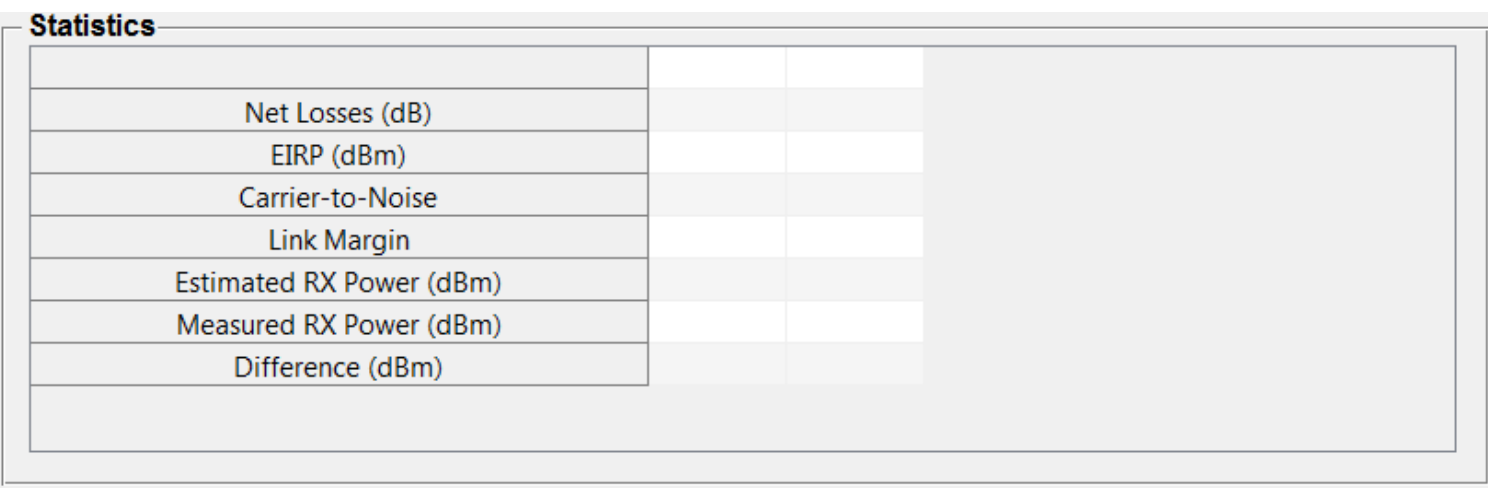

Figure 15. Output Statistics Panel Which Is Filled In Every Time A Link-Budet Calculation Is Run

\subsubsection{Link Budget and Analysis}

Now that the coupled model has been presented, it would be useful to demonstrate the functionality by performing a sample link budget calculation. In order to perform the calculation, user determined parameters must be input to the GUI. For each panel on the GUI, every pushbutton must be selected. If no values are saved, the GUI will warn the user and send default values to the link-budget code. For example, the channel specifications push-button was selected, then canceled out. The warning message that would pop up can be seen in Figure 16. If the user 
attempts to run the link-budget calculation without all of the completion boxes checked, designating that input data is saved, then an error message will pop up as seen in Figure 17. For the sake of this example calculation, default values will be used. The input data for the GUI at 10 GHz is shown in Table 1 .

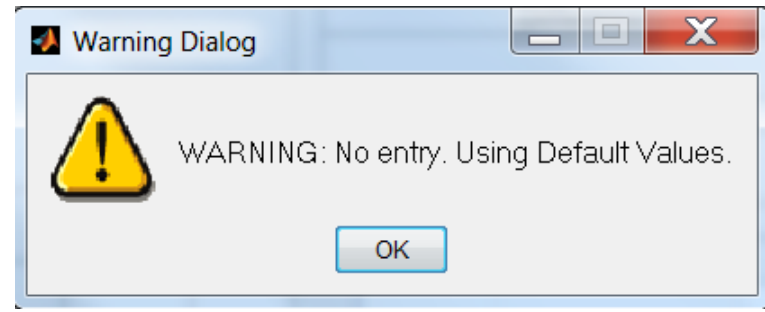

Figure 16. Typical Warning Box That Would Pop-up When Either No Entries Were Saved Or The Data Fields Were Not Correctly Filled Out

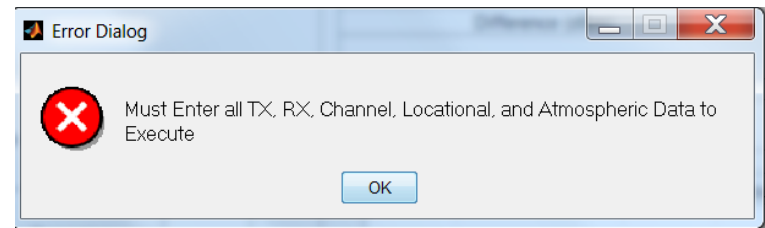

\section{Figure 17. Typical Error Box That Would Pop-up When Program Execution Is Attempted Without The Necessary Input Data}

The majority of the data that is conveyed in Table 2 are typical output data sets. This data was manually extracted for more convenient presentation. The majority of losses seen on the right will not be present in the GUI output sections, but can be extracted easily. The primary factor of interest is the estimated received power. From there, the user can decide whether the power is sufficient or not. If it is sufficient, then it requires no additional efforts. If the received power is insufficient, then the values must be tweaked and tradeoffs must be made in order to achieve the required received power. 
Table 1. Input Parameters for a Sample Link-Budget Calculation at $10 \mathrm{GHz}$

\begin{tabular}{|c|c|c|c|}
\hline \multicolumn{2}{|c|}{ Channel Specifications } & \multicolumn{2}{|c|}{ Transmitting System } \\
\hline Frequency & $10 \mathrm{GHz}$ & Antenna Gain & $12.2 \mathrm{dBi}$ \\
\hline Transmit Power & $25 \mathrm{dBm}$ & Largest Aperture Dimension & $.24 \mathrm{~m}$ \\
\hline $\begin{array}{l}\text { Transmitter } \\
\text { Height }\end{array}$ & $5280 \mathrm{ft}$. & Antenna Efficiency & 0.5 \\
\hline Receiver Height & $5281 \mathrm{ft}$. & Pre-Amp Cable Losses & $3 \mathrm{~dB}$ \\
\hline \multirow[t]{3}{*}{ Channel Length } & $.046 \mathrm{~km}$ & Post- Amp Cable Losses & $3 \mathrm{~dB}$ \\
\hline & & Amplifier Gain & $44 \mathrm{~dB}$ \\
\hline & & $\mathrm{P} 1$ & $41 \mathrm{dBm}$ \\
\hline \multicolumn{4}{|c|}{ Site Location } \\
\hline Longitude & 254 & & \\
\hline Latitude & 35 & \multicolumn{2}{|c|}{ Transmitting System } \\
\hline \multicolumn{2}{|c|}{ Atmosphere } & Antenna Gain & $12.2 \mathrm{dBi}$ \\
\hline Temperature & $22 \mathrm{C}$ & Largest Aperture Dimension & $.24 \mathrm{~m}$ \\
\hline Pressure & $1024 \mathrm{hPa}$ & Antenna Efficiency & 0.5 \\
\hline Humidity & $30 \%$ & Cable Losses & $3 \mathrm{~dB}$ \\
\hline
\end{tabular}


Table 2. Sample Calculation at $10 \mathrm{GHz}$ Performed by the Coupled Model

\begin{tabular}{|c|c|c|c|c|c|}
\hline \multicolumn{7}{|c|}{ LINK BUDGET at 10 GHz and 46 m } \\
\hline Transmit Power & 25 & $\mathrm{dBm}$ & & & \\
\hline Transmit Antenna Gain & 12.2 & $\mathrm{dBi}$ & & & \\
\hline Feed Losses & 3 & $\mathrm{~dB}$ & Free Space Path Loss & 85.7029 & $\mathrm{~dB}$ \\
\hline EIRP & 38 & $\mathrm{dBm}$ & Polarization Losses & 3.0103 & $\mathrm{~dB}$ \\
\hline & & & Gaseous Losses & 0.0005905 & $\mathrm{~dB}$ \\
\hline Receive Antenna Gain & 12.2 & $\mathrm{dBi}$ & Scintillation Losses & 0.0017 & $\mathrm{~dB}$ \\
\hline Feed Losses & 3 & $\mathrm{~dB}$ & Rain Losses & 0 & $\mathrm{~dB}$ \\
\hline Received Power & -41.5156 & $\mathrm{dBm}$ & Clouds \& Fog Losses & 0 & $\mathrm{~dB}$ \\
\hline & & & Dust Losses & 0 & $\mathrm{~dB}$ \\
\hline System Noise Power & -177 & $\mathrm{dBW}$ & Misc. Losses & 0 & $\mathrm{~dB}$ \\
\hline C/N & 135.4844 & $\mathrm{~dB}$ & Net Power Losses & 88.7156 & $\mathrm{~dB}$ \\
\hline C/N Threshold & -20 & $\mathrm{dBm}$ & & & \\
\hline Link Margin & 115.4844 & $\mathrm{~dB}$ & & & \\
\hline
\end{tabular}

In this case, the predicted received power is $-41.5156 \mathrm{dBm}$ whereas the noise floor is set at $-177 \mathrm{dBm}$. It is obvious that the current system is more than sufficient since the received power has a high carrier-to-noise ratio, and the conservative $20 \mathrm{~dB}$ link margin requirement is adequately met. Unfortunately, the noise floor will almost never sit at -177 dBm.

In the scenario where the noise floor was up to $-52 \mathrm{dBm}$, the system would require modification. This would result in the link margin becoming negative by about $9 \mathrm{dBm}$. Some system changes that could be made are: choosing higher gain antennas, choosing low-loss cables, transmit at a higher power, add a high power amplifier to the transmitting system, etc. This was just a simple example of how the GUI could be used practically. 


\subsection{Model Validation Approach}

Every model needs to be properly tested for it to be considered a reliable design tool (i.e., verification and validation). The vast majority of the individual models that the coupled model is comprised of have been investigated extensively. However, a model uniting them all has not.

The approach to test the developed link-budget model was relatively simple. The first phase would be to take simple line-of-sight (LOS) free-space measurements, and determine if the predicted calculations match the data well. If the system is truly LOS, then the losses experienced should be solely propagation losses (assuming all other losses are accounted for exactly). This would prove that the model can withstand the typical environment without the addition of excess attenuation. Depending on the prediction method's accuracy, the next step would either be to improve the model, and/or attempt propagation measurements in a different scenario.

Once enough data sets are collected and the model has proven to be accurate, the next phase would be to introduce excess attenuation to the system. The attenuation of most interest would be rain, since it is the one that most severely impacts satellite communication systems.

Again, after sufficient data is collected and the model is deemed accurate, the third phase would be to increase the frequency in the $\mathrm{V}$ and $\mathrm{W}$-bands to begin model characterization. In these frequency bands, extensive data would need to be collected, since propagation impairments in these bands are relatively unpredictable.

\subsection{Experiment 1 - Measuring Propagation Losses}

The first experiment had a primary goal of transmitting and receiving a signal across a channel in a practical situation. In addition, the secondary goal was to compare the measured results with the designed propagation model and determine the error. Based on the results, the decision would be made to either continue further development of the models, or seek an alternate route. Therefore, it was imperative that the results be fairly accurate while allowing measurable losses through the air.

The plan was to create a point-to-point link in which the power loss due to propagation could be directly measured, since other impairments would be minimized. This setup is also convenient since it would mimic a future system operating in the $\mathrm{V}$ and $\mathrm{W}$-bands, which would

Approved for Public Release; Distribution is Unlimited. 
need to be a LOS system. After this was decided, the next decision was to use a frequency in the $\mathrm{X}$-band. This decision was made based on two aspects:

1. X-band frequencies are well characterized and propagation impairments are well known, and

2. X-band equipment (e.g. transmitter and antennas) was readily available.

It was thought that if the model could predict power that accurately matches the clear-sky measurements, then an initial baseline would be set. The consequence of operating at such a distant frequency though is that the propagation impairments and system performance cannot necessarily be linearly scaled to higher frequencies. Since the primary goal was only to create a link, this issue was left alone.

Following the macroscopic system specifications, the next decision was to implement the experiment while mitigating as many external loss sources as possible. In order to minimize the effect of multipath and ground reflections as much possible, the transmitter and receiver were placed on the roofs of neighboring buildings at the University of New Mexico (UNM). The channel was specifically setup between the roofs of UNM's Electrical \& Computer Engineering and Centennial Engineering Buildings as can be seen in Figure 18.

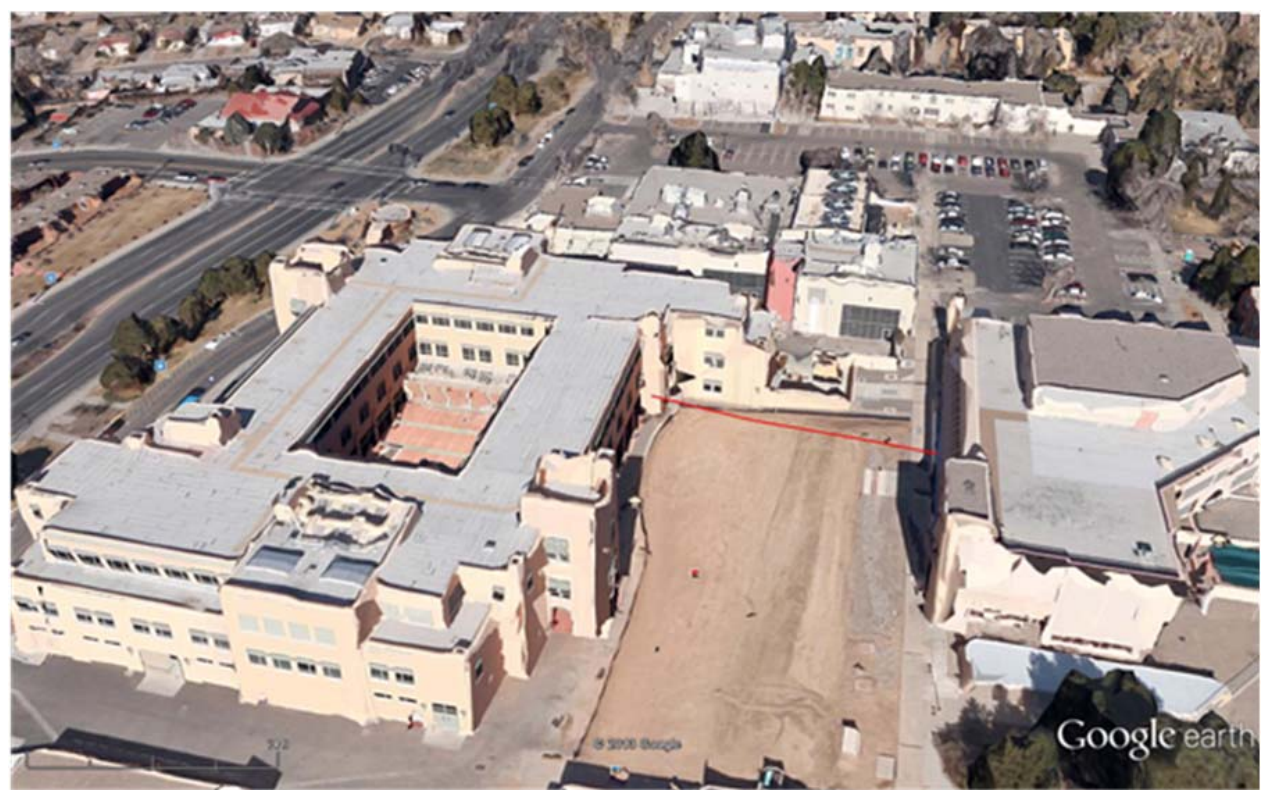

Figure 18. An Overview of the First Propagation Experiment at $10 \mathrm{GHz}$ 
Originally, it was thought that a power sweep would be beneficial to the work. After obtaining a better idea of what the overall goal was, that idea seemed to be unnecessary, although it was still performed in the first experiment. An overview of the experimental setup can be seen in Figure 18. An actual picture from the experiment is shown in Figure 19. In order to achieve the experimental goals, specific equipment that met the requirements was utilized. The equipment used is split into two categories below; transmitting system and receiving system. These are described below.

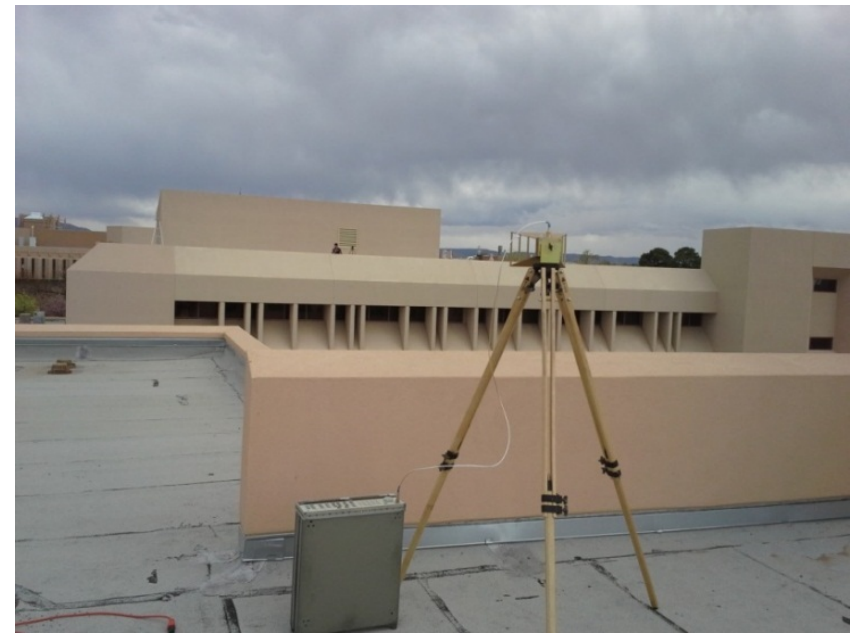

Figure 19. Actual Footage of the First Experiment at $10 \mathrm{GHz}$

\subsubsection{Transmitting System}

- Signal Generator - HP 83752B Synthesized Sweeper

o Capable of generating continuous waves (CWs) with transmit power at 25 $\mathrm{dBm}$

o Frequency Range from $10 \mathrm{MHz}$ to $20 \mathrm{GHz}$

- Transmitting Antenna - SAS-571 Double Ridge Guide Horn Antenna

o Wideband antenna $(700 \mathrm{MHz}-18 \mathrm{GHz})$

o Approximately $12.2 \mathrm{dBi}$ of gain at $10 \mathrm{GHz}$

- Sub-Miniature version A (SMA) $3.5 \mathrm{~mm}$ connector cable 


\subsubsection{Receiving System}

- Receiver - N9030A PXA Signal Analyzer

o Capable of measuring signal levels near $-159 \mathrm{dBm}$ at $10 \mathrm{GHz}$.

o Can handle frequencies up to $110 \mathrm{GHz}$.

- Receiving Antenna - SAS-571 Double Ridge Guide Horn Antenna

o Wideband antenna $(700 \mathrm{MHz}-18 \mathrm{GHz})$

o Approximately $12.2 \mathrm{dBi}$ of gain at $10 \mathrm{GHz}$

- SMA 3.5mm connector cable

\subsubsection{Specifications}

Many different data parameters such as meteorological data and channel specifications were needed in order to accurately predict the received power. At the time, local rather than site specific meteorological data was utilized. The receiver location and channel length were determined through the Google Earth program, since physical measurements could not be taken. The antenna heights and aperture dimensions were measured by hand. The antenna efficiency of 0.5 tends to be a common conservative value used by most. The cable losses were measured after the experiment using a network analyzer. Although not listed here, the model incorporates a $3 \mathrm{~dB}$ loss due to polarization mismatch, which is also a conservative, but a practical value for this. The input data for the GUI is shown in Table 3. As mentioned previously, a power sweep was performed at $10 \mathrm{GHz}$ during this experiment because it was originally thought to be beneficial. Since this was proved to be unnecessary, it is not mentioned in Table 3. For further reference, the output powers tested were 11, 14, 17, 20, and $23 \mathrm{dBm}$. The parameters in Table 3 were input into the coupled model and a simulation was run. The expected performance is conveyed in Table 4. Since then, modifications have been made to the link-budget model, and the original predicted data slightly differed. This is conveyed in Table 5 . 
Table 3. Input Parameters and Link-Budget Calculations for the First Experiment

\begin{tabular}{|c|c|c|c|}
\hline \multicolumn{2}{|c|}{ Channel Specifications } & \multicolumn{2}{|c|}{ Transmitting System } \\
\hline Frequency & $10 \mathrm{GHz}$ & Antenna Gain & $12.2 \mathrm{dBi}$ \\
\hline Transmit Power & $23 \mathrm{dBm}$ & $\begin{array}{l}\text { Largest Aperture } \\
\text { Dimension }\end{array}$ & $0.24 \mathrm{~m}$ \\
\hline Transmitter Height & $5201 \mathrm{ft}$. & Antenna Efficiency & 0.5 \\
\hline Receiver Height & $5205 \mathrm{ft}$. & Cable Losses & -3.217 \\
\hline Channel Length & $0.0467 \mathrm{~km}$ & & \\
\hline \multicolumn{2}{|c|}{ Site Location } & & \\
\hline Longitude & 253.63 & & \\
\hline Latitude & 35.05 & \multicolumn{2}{|c|}{ Receiving System } \\
\hline \multicolumn{2}{|c|}{ Atmosphere } & Antenna Gain & $12.2 \mathrm{dBi}$ \\
\hline Temperature & $22 \mathrm{C}$ & $\begin{array}{l}\text { Largest Aperture } \\
\text { Dimension }\end{array}$ & $0.24 \mathrm{~m}$ \\
\hline Pressure & $1024 \mathrm{hPa}$ & Antenna Efficiency & 0.5 \\
\hline Humidity & $30 \%$ & Cable Losses & -7.71289 \\
\hline
\end{tabular}

Table 4. Output Data from the Link-Budget Model for the First Experiment

\begin{tabular}{|l|c|c|l|c|c|}
\hline \multicolumn{7}{|c|}{ LINK BUDGET at 10 GHz and 46.7 m } \\
\hline Transmit Power & 23 & $\mathrm{dBm}$ & Receive Antenna Gain & 12.2 & $\mathrm{dBi}$ \\
\hline Transmit Antenna Gain & 12.2 & $\mathrm{dBi}$ & Feed Losses & 7.71289 & $\mathrm{~dB}$ \\
\hline Feed Losses & 3.217 & $\mathrm{~dB}$ & Received Power & -52.377 & $\mathrm{dBm}$ \\
\hline EIRP & 31.983 & $\mathrm{dBm}$ & & & \\
\hline & & & System Noise Power & -84.773 & $\mathrm{dBW}$ \\
\hline Free Space Path Loss & 85.8341 & $\mathrm{~dB}$ & C/N & 32.396 & $\mathrm{~dB}$ \\
\hline Gaseous Loss & 0.00069 & $\mathrm{~dB}$ & C/N Threshold & -20 & $\mathrm{dBm}$ \\
\hline Net Power Losses & 88.8467 & $\mathrm{~dB}$ & Link Margin & 12.396 & $\mathrm{~dB}$ \\
\hline
\end{tabular}


Table 5. Output Data Post Data Analysis Discussion

\begin{tabular}{|l|c|c|l|c|c|}
\hline \multicolumn{7}{|c|}{ LINK BUDGET at 10 GHz and 46.7 m } \\
\hline Transmit Power & 23 & $\mathrm{dBm}$ & Receive Antenna Gain & 12.2 & $\mathrm{dBi}$ \\
\hline Transmit Antenna Gain & 12.2 & $\mathrm{dBi}$ & Feed Losses & 7.71289 & $\mathrm{~dB}$ \\
\hline Feed Losses & 3.217 & $\mathrm{~dB}$ & Received Power & -52.377 & $\mathrm{dBm}$ \\
\hline EIRP & 31.983 & $\mathrm{dBm}$ & & & \\
\hline & & & System Noise Power & -84.773 & $\mathrm{dBW}$ \\
\hline Free Space Path Loss & 85.8341 & $\mathrm{~dB}$ & $\mathrm{C} / \mathrm{N}$ & 32.396 & $\mathrm{~dB}$ \\
\hline Gaseous Loss & 0.00069 & $\mathrm{~dB}$ & C/N Threshold & -20 & $\mathrm{dBm}$ \\
\hline Net Power Losses & 88.8467 & $\mathrm{~dB}$ & Link Margin & 12.396 & $\mathrm{~dB}$ \\
\hline
\end{tabular}

\subsection{Experiment 2 - Quantifying Accuracy in an Anechoic Chamber}

Following the discovery of a rain simulator, further out-door measurements were desired. Prior to working with the rain simulator, it was desired to quantify the model's accuracy without influence of any external sources (multipath and outside noise). This was important, since the specific attenuation expected at $10 \mathrm{GHz}$ is only $0.8134 \mathrm{~dB} / \mathrm{km}$. This specific attenuation was troublesome since the rain simulator only spanned a maximum distance of 59 feet. In order to eliminate the vast majority of noise sources and multipath, an anechoic chamber was needed. The large-scale anechoic chamber operated by the Air Force Research Laboratories was used for this.

The additional data taken were measurements at several frequencies above $10 \mathrm{GHz}$. To satisfy this requirement, the frequencies of 20,30 , and $40 \mathrm{GHz}$ were also investigated. These frequencies are much easier to detect losses with since they are much more sensitive to exterior attenuation than the lower X-band frequencies. At this time, the use of higher frequency channels and equipment were not thought to be used in the field test, but rather just for model verification. In the anechoic chamber, several received power measurements were taken at all four frequencies to determine propagation losses. The experimental setup can be vaguely seen in Figure 20. 


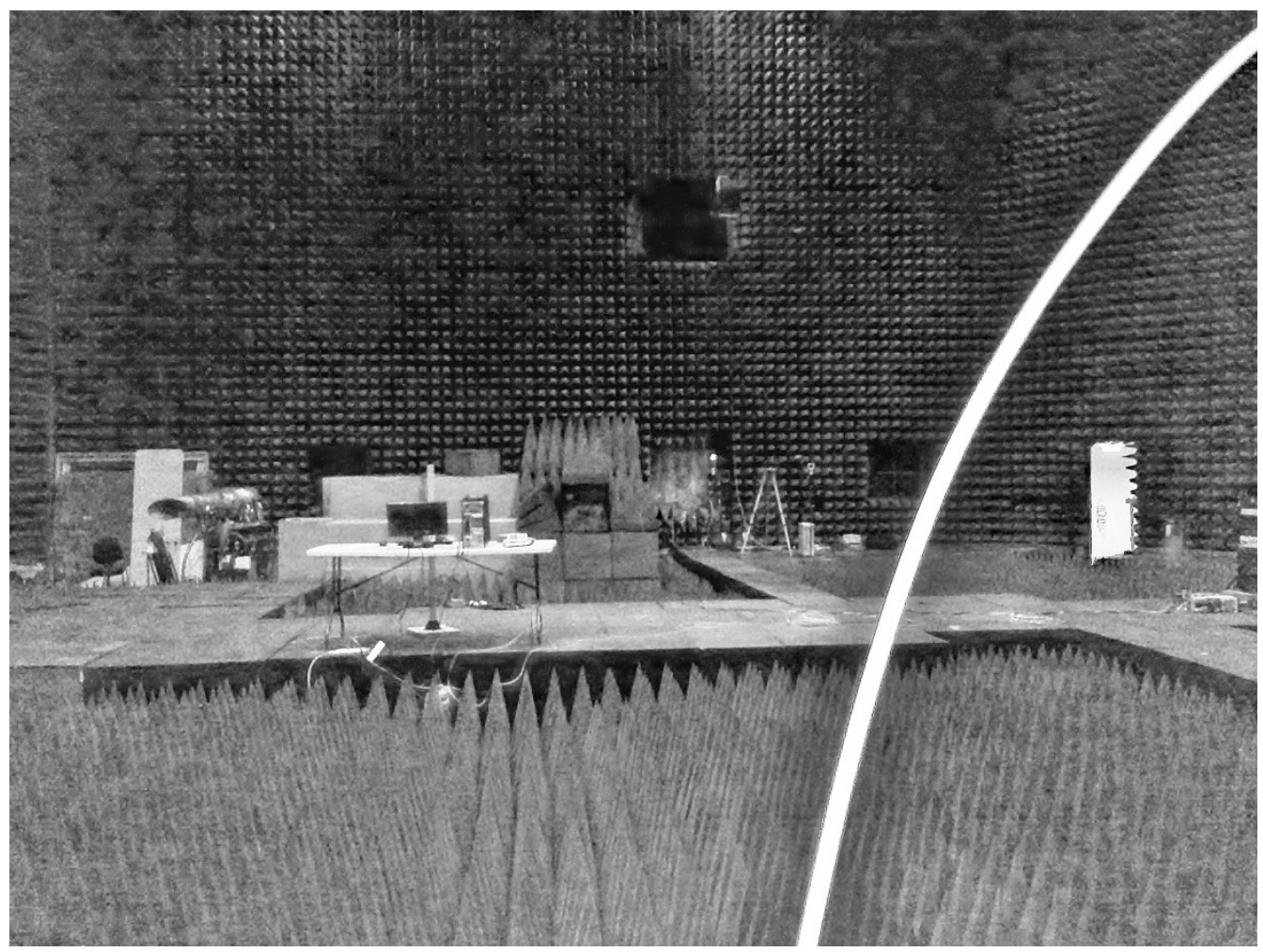

Figure 20. Actual Footage of the Experiment at 10, 20, 30, and $40 \mathrm{GHz}$ within the Anechoic Chamber

In order to achieve the experimental goals, specific equipment that met the requirements was utilized. In this case, two different communication links were created; one utilizing the original test equipment for $10 \mathrm{GHz}$, and another for the other three frequencies utilizing equipment rated for higher frequencies. The equipment used is split into four categories; a transmitting system and receiving system for $10 \mathrm{GHz}$, and a transmitting system and receiving system for 20, 30, $40 \mathrm{GHz}$. These are seen below.

\subsubsection{GHz Transmitting System}

- Signal Generator - HP 83752B Synthesized Sweeper

o Capable of generating continuous waves (CWs) with transmit power at 15 $\mathrm{dBm}$

o Frequency Range from $10 \mathrm{MHz}$ to $20 \mathrm{GHz}$

- Transmitting Antenna - SAS-571 Double Ridge Guide Horn Antenna 
o Wideband antenna (700 MHz $-18 \mathrm{GHz})$

o Approximately $12.2 \mathrm{dBi}$ of gain at $10 \mathrm{GHz}$

- SMA 3.5mm connector cable

\subsubsection{GHz Receiving System}

- Receiver - N9030A PXA Signal Analyzer

o Capable of measuring signal levels near $-159 \mathrm{dBm}$ at $10 \mathrm{GHz}$.

o Can handle frequencies up to $110 \mathrm{GHz}$

- Receiving Antenna - SAS-571 Double Ridge Guide Horn Antenna

o Wideband antenna $(700 \mathrm{MHz}-18 \mathrm{GHz})$

o Approximately $12.2 \mathrm{dBi}$ of gain at $10 \mathrm{GHz}$

- SMA 3.5mm connector cable

\subsubsection{0, 30, and 40 GHz Transmitting System}

- $\quad$ Signal Generator - Agilent E8257D

o Capable of generating continuous waves (CWs) with transmit power at 15 $\mathrm{dBm}$

o Maximum Operating Frequency - $50 \mathrm{GHz}$

- Transmitting Antenna - AH-840 Horn Antenna

○ Broadband antenna $(18-40 \mathrm{GHz})$

o Approximately 21.63, 23.65, and $21.05 \mathrm{dBi}$ of gain at 20,30 , and $40 \mathrm{GHz}$ respectively

- SMA K-type connector cables

\subsubsection{0, 30, \& 40 GHz Receiving System}

- Receiver - N9030A PXA Signal Analyzer

o Capable of measuring signal levels near $-159 \mathrm{dBm}$ at $10 \mathrm{GHz}$.

o Can handle frequencies up to $110 \mathrm{GHz}$

- Receiving Antenna - AH-840 Horn Antenna

o Broadband antenna $(18-40 \mathrm{GHz})$ 
o Approximately $21.63,23.65$, and $21.05 \mathrm{dBi}$ of gain at 20,30 , and $40 \mathrm{GHz}$ respectively

- SMA K-type connector cables

\subsubsection{Specifications}

The antenna efficiency of 0.5 was used in the predictive calculation for all four frequencies. The cable losses were only predicted in this case, since the equipment used could not be removed from the facility. The predictions were made according to manufacturer specifications. In this particular case, the only anticipated losses that were not included in the model were attributed to pointing losses. The input data for the GUI at $10 \mathrm{GHz}$ and $20-40 \mathrm{GHz}$ are shown in Table 6 and Table 7, respectively. The expected performance predictions are displayed in Table 8, Table 9, Table 10, and Table 11.

Table 6. Link-Budget Calculation at $10 \mathrm{GHz}$

\begin{tabular}{|c|c|c|c|c|c|}
\hline \multicolumn{7}{|c|}{ LINK BUDGET at 10 GHz and 19.431 m } \\
\hline Transmit Power & 15 & $\mathrm{dBm}$ & & & \\
\hline Transmit Antenna Gain & 12.2 & $\mathrm{dBi}$ & & \\
\hline Feed Losses & 0.87 & $\mathrm{~dB}$ & Free Space Path Loss & 78.2177 & $\mathrm{~dB}$ \\
\hline EIRP & 26.33 & $\mathrm{dBm}$ & Polarization Losses & 3.0103 & $\mathrm{~dB}$ \\
\hline Receive Antenna Gain & 12.2 & $\mathrm{dBi}$ & Scintillation Losses & 0.0016 & $\mathrm{~dB}$ \\
\hline Feed Losses & 0.87 & $\mathrm{~dB}$ & Rain Losses & 0 & $\mathrm{~dB}$ \\
\hline Received Power & -43.5698 & $\mathrm{dBm}$ & Clouds \& Fog Losses & 0 & $\mathrm{~dB}$ \\
\hline & & & Dust Losses & 0 & $\mathrm{~dB}$ \\
\hline System Noise Power & -177 & $\mathrm{dBW}$ & Misc. Losses & 0 & $\mathrm{~dB}$ \\
\hline C/N & 133.4302 & $\mathrm{~dB}$ & Net Power Losses & 81.2298 & $\mathrm{~dB}$ \\
\hline C/N Threshold & -20 & $\mathrm{dBm}$ & & & \\
\hline Link Margin & 113.4302 & $\mathrm{~dB}$ & & & \\
\hline
\end{tabular}


Table 7. Input Parameters for Link-Budget Calculations at 20-40 GHz

\begin{tabular}{|c|c|c|c|}
\hline \multicolumn{2}{|c|}{ Channel Specifications } & \multicolumn{2}{|c|}{ Transmitting System } \\
\hline Frequency & $20,30,40 \mathrm{GHz}$ & $\begin{array}{l}\text { Antenna Gain } \\
(20,30,40 \mathrm{GHz})\end{array}$ & $21.63,23.65,21.05 \mathrm{dBi}$ \\
\hline Transmit Power & $15 \mathrm{dBm}$ & $\begin{array}{l}\text { Largest Aperture } \\
\text { Dimension }\end{array}$ & $0.06858 \mathrm{~m}$ \\
\hline Transmitter Height & $5235.8125 \mathrm{ft}$. & Antenna Efficiency & 0.5 \\
\hline Receiver Height & $5376.785 \mathrm{ft}$. & $\begin{array}{l}\text { Cable Losses } \\
(20,30,40 \mathrm{GHz})\end{array}$ & $3.39,4.33,7.32 \mathrm{~dB}$ \\
\hline Channel Length & $0.019431 \mathrm{~km}$ & & \\
\hline \multicolumn{2}{|c|}{ Site Location } & & \\
\hline Longitude & 253.64 & & \\
\hline Latitude & 35.2 & \multicolumn{2}{|c|}{ Receiving System } \\
\hline \multicolumn{2}{|c|}{ Atmosphere } & Antenna Gain & $12.2 \mathrm{dBi}$ \\
\hline Temperature & $21.1111 \mathrm{C}$ & $\begin{array}{l}\text { Largest Aperture } \\
\text { Dimension }\end{array}$ & $0.06858 \mathrm{~m}$ \\
\hline Pressure & $1009.34 \mathrm{hPa}$ & Antenna Efficiency & 0.5 \\
\hline Humidity & $26 \%$ & $\begin{array}{l}\text { Cable Losses } \\
(20,30,40 \mathrm{GHz})\end{array}$ & $2.35,2.83,4.86 \mathrm{~dB}$ \\
\hline
\end{tabular}


Table 8. Link-Budget Calculation at $10 \mathrm{GHz}$

\begin{tabular}{|l|c|c|l|c|c|}
\hline \multicolumn{7}{|c|}{ LINK BUDGET at 10 GHz and 19.431 m } \\
\hline Transmit Power & 15 & $\mathrm{dBm}$ & & & \\
\hline Transmit Antenna Gain & 12.2 & $\mathrm{dBi}$ & & & \\
\hline Feed Losses & 0.87 & $\mathrm{~dB}$ & Free Space Path Loss & 78.2177 & $\mathrm{~dB}$ \\
\hline EIRP & 26.33 & $\mathrm{dBm}$ & Polarization Losses & 3.0103 & $\mathrm{~dB}$ \\
\hline & & & Gaseous Losses & 0.000245 & $\mathrm{~dB}$ \\
\hline Receive Antenna Gain & 12.2 & $\mathrm{dBi}$ & Scintillation Losses & 0.0016 & $\mathrm{~dB}$ \\
\hline Feed Losses & 0.87 & $\mathrm{~dB}$ & Rain Losses & 0 & $\mathrm{~dB}$ \\
\hline Received Power & -43.5698 & $\mathrm{dBm}$ & Clouds \& Fog Losses & 0 & $\mathrm{~dB}$ \\
\hline & & & Dust Losses & 0 & $\mathrm{~dB}$ \\
\hline System Noise Power & -177 & $\mathrm{dBW}$ & Misc. Losses & 0 & $\mathrm{~dB}$ \\
\hline C/N & 133.4302 & $\mathrm{~dB}$ & Net Power Losses & 81.2298 & $\mathrm{~dB}$ \\
\hline C/N Threshold & -20 & $\mathrm{dBm}$ & & & \\
\hline Link Margin & 113.4302 & $\mathrm{~dB}$ & & & \\
\hline
\end{tabular}


Table 9. Link-Budget Calculation at $20 \mathrm{GHz}$

\begin{tabular}{|l|c|c|l|c|c|}
\hline \multicolumn{7}{|c|}{ LINK BUDGET at 20 GHz and 19.431 m } \\
\hline Transmit Power & 15 & $\mathrm{dBm}$ & & & \\
\hline Transmit Antenna Gain & 21.63 & $\mathrm{dBi}$ & & & \\
\hline Feed Losses & 3.39 & $\mathrm{~dB}$ & Free Space Path Loss & 84.2383 & $\mathrm{~dB}$ \\
\hline EIRP & 33.24 & $\mathrm{dBm}$ & Polarization Losses & 3.0103 & $\mathrm{~dB}$ \\
\hline & & & Gaseous Losses & 0.0019 & $\mathrm{~dB}$ \\
\hline Receive Antenna Gain & 21.63 & $\mathrm{dBi}$ & Scintillation Losses & 0.0024 & $\mathrm{~dB}$ \\
\hline Feed Losses & 2.35 & $\mathrm{~dB}$ & Rain Losses & 0 & $\mathrm{~dB}$ \\
\hline Received Power & -34.7328 & $\mathrm{dBm}$ & Clouds \& Fog Losses & 0 & $\mathrm{~dB}$ \\
\hline & & & Dust Losses & 0 & $\mathrm{~dB}$ \\
\hline System Noise Power & -177 & $\mathrm{dBW}$ & Misc. Losses & 0 & $\mathrm{~dB}$ \\
\hline C/N & 142.2672 & $\mathrm{~dB}$ & Net Power Losses & 87.2528 & $\mathrm{~dB}$ \\
\hline C/N Threshold & -20 & $\mathrm{dBm}$ & & & \\
\hline Link Margin & 122.2672 & $\mathrm{~dB}$ & & & \\
\hline
\end{tabular}


Table 10. Link-Budget Calculation at $30 \mathrm{GHz}$

\begin{tabular}{|l|c|c|l|c|c|}
\hline \multicolumn{7}{|c|}{ LINK BUDGET at 30 GHz and 19.431 m } \\
\hline Transmit Power & 15 & $\mathrm{dBm}$ & & & \\
\hline Transmit Antenna Gain & 23.65 & $\mathrm{dBi}$ & & & \\
\hline Feed Losses & 4.33 & $\mathrm{~dB}$ & Free Space Path Loss & 87.7601 & $\mathrm{~dB}$ \\
\hline EIRP & 34.32 & $\mathrm{dBm}$ & Polarization Losses & 3.0103 & $\mathrm{~dB}$ \\
\hline & & & Gaseous Losses & 0.0016 & $\mathrm{~dB}$ \\
\hline Receive Antenna Gain & 23.65 & $\mathrm{dBi}$ & Scintillation Losses & 0.003 & $\mathrm{~dB}$ \\
\hline Feed Losses & 2.83 & $\mathrm{~dB}$ & Rain Losses & 0 & $\mathrm{~dB}$ \\
\hline Received Power & -35.65 & $\mathrm{dBm}$ & Clouds \& Fog Losses & 0 & $\mathrm{~dB}$ \\
\hline & & & Dust Losses & 0 & $\mathrm{~dB}$ \\
\hline System Noise Power & -177 & $\mathrm{dBW}$ & Misc. Losses & 0 & $\mathrm{~dB}$ \\
\hline C/N & 141.365 & $\mathrm{~dB}$ & Net Power Losses & 90.775 & $\mathrm{~dB}$ \\
\hline C/N Threshold & -20 & $\mathrm{dBm}$ & & & \\
\hline Link Margin & 121.365 & $\mathrm{~dB}$ & & & \\
\hline
\end{tabular}


Table 11. Link-Budget Calculation at $40 \mathrm{GHz}$

\begin{tabular}{|l|c|c|l|c|c|}
\hline \multicolumn{5}{|c|}{ LINK BUDGET at 40 GHz and 19.431 m } \\
\hline Transmit Power & 15 & $\mathrm{dBm}$ & & & \\
\hline Transmit Antenna Gain & 21.05 & $\mathrm{dBi}$ & & & \\
\hline Feed Losses & 7.32 & $\mathrm{~dB}$ & Free Space Path Loss & 90.2589 & $\mathrm{~dB}$ \\
\hline EIRP & & $\mathrm{dBm}$ & Polarization Losses & 3.0103 & $\mathrm{~dB}$ \\
\hline & & & Gaseous Losses & 0.0022 & $\mathrm{~dB}$ \\
\hline Receive Antenna Gain & 21.05 & $\mathrm{dBi}$ & Scintillation Losses & 0.0035 & $\mathrm{~dB}$ \\
\hline Feed Losses & 4.86 & $\mathrm{~dB}$ & Rain Losses & 0 & $\mathrm{~dB}$ \\
\hline Received Power & & $\mathrm{dBm}$ & Clouds \& Fog Losses & 0 & $\mathrm{~dB}$ \\
\hline & & & Dust Losses & 0 & $\mathrm{~dB}$ \\
\hline System Noise Power & -177 & $\mathrm{dBW}$ & Misc. Losses & 0 & $\mathrm{~dB}$ \\
\hline C/N & & $\mathrm{dB}$ & Net Power Losses & 93.2749 & $\mathrm{~dB}$ \\
\hline C/N Threshold & -20 & $\mathrm{dBm}$ & & & \\
\hline Link Margin & & $\mathrm{dB}$ & & & \\
\hline
\end{tabular}

\subsection{Experiment 3 - Quantifying Model Accuracy with Rain Impairment}

Once it was determined that the model was tested adequately under clear-sky conditions, it was decided to further examine the model's capabilities by adding precipitation into the equation. As mentioned previously, a UNM rain simulator was discovered and utilized to validate the coupled model with precipitation attenuation. The rain simulator was designed to simulate monsoon-like rainfall to monitor vegetation growth and soil variations on a 9 x $14 \mathrm{~m}$ plot. For their purposes, the rainfall had to be uniform throughout the plot, and the distribution lines had to be high enough to allow the rain drop to achieve a near natural velocity. They achieved the uniform distribution in their design by utilizing rain drop quality sprinkler heads and creating an overlapping circular rain patterns. This can be seen in Figure 21. 


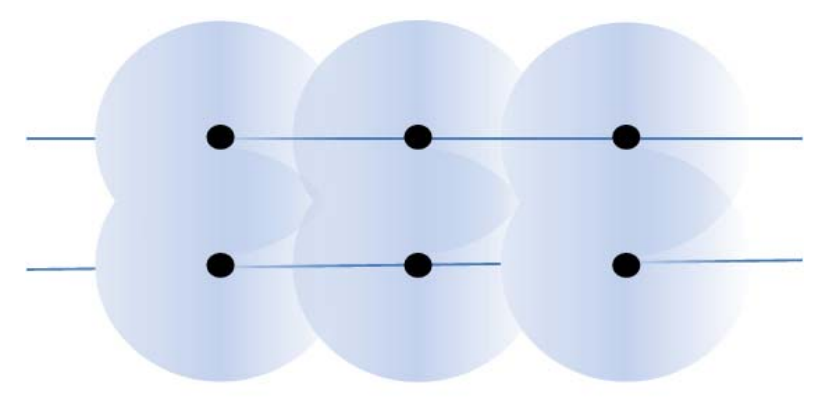

\section{Figure 21. Top View Animation of the Rain Simulator Exemplifying the Uniform Distribution of Rainfall}

Throughout the summer, the Biologist group emulated $20 \mathrm{~mm} /$ hour and $60 \mathrm{~mm} /$ hour rain events. Although the simulators are unable to operate for an entire hour, the rain rates were provided by the Biologist group. It was desired to perform several experiments to acquire a decent amount of data for analysis. The plan was then to perform two experiments with rainfall at $20 \mathrm{~mm} /$ hour, and one experiment with rainfall at $60 \mathrm{~mm} /$ hour. More would have been desired, but the experiment schedule for the Biologists was coming to an end. This experiment was to be the first experiment with rainfall at $20 \mathrm{~mm} /$ hour.

Unfortunately, the rainfall zone is relatively small, spanning over a $14 \times 9 \mathrm{~m}$ plot. Due to the limited measurement area, exploiting the space was important. It was decided to have the wave propagate diagonally across the rain area in order to increase the distance in which the signal was interacting with rain. This would also increase the rain's impact on the signal.

Another important part of the experiment to be mentioned was the approximate time of the execution. Since the rain simulator is located outside, it is subject to uncontrollable forces like wind. Wind has a negative impact on the rain simulator, since it tends to redirect rainfall outside the measurement area resulting in non-uniform rainfall. In an attempt to eliminate wind effects, the experiments were scheduled to occur from 8:00 AM to 9:00 AM. The Biology team stated that wind speeds are generally less intense in the early mornings during the summer.

Three sets of ten measurements were to be taken during this experiment; a set before rainfall, a set during rainfall, and a set following the rainfall. The reason for multiple measurement sets was that multipath was expected to occur during the experiment, and the effect would change throughout. It was believed that it would change throughout because the ground, 
which was in close proximity to the antennas, would accumulate the rapidly falling rain water at the surface before it could absorb it.

To summarize, the goal of this experiment was to measure the propagation losses across the plot at $10 \mathrm{GHz}$ prior to, during, and following the rain simulation. The hope was that noticeable losses would be measured and the results would match the link-budget model. As in the first experiment, a single $10 \mathrm{GHz}$ communications link was setup to test the model. The equipment used was split into three categories below; a transmitting system and a receiving system for $10 \mathrm{GHz}$, and miscellaneous equipment. These are described below.

\subsection{1 $10 \mathrm{GHz}$ Transmitting System}

- $\quad$ Signal Generator - HP 83752B Synthesized Sweeper

o Capable of generating CWs with transmit power at $15 \mathrm{dBm}$

o Frequency Range from $10 \mathrm{MHz}$ to $20 \mathrm{GHz}$

- Transmitting Antenna - SAS-571 Double Ridge Guide Horn Antenna

o Wideband antenna $(700 \mathrm{MHz}-18 \mathrm{GHz})$

o Approximately $12.2 \mathrm{dBi}$ of gain at $10 \mathrm{GHz}$

- X-Band Amplifier by Microsemi

o Provides approximately $42 \mathrm{~dB}$ of gain

o Has a saturation power at approximately $40.3 \mathrm{dBm}$

- 2 SMA $3.5 \mathrm{~mm}$ connector cables

\subsubsection{GHz Receiving System}

- Receiver - N9030A PXA Signal Analyzer

o Capable of measuring signal levels near $-159 \mathrm{dBm}$ at $10 \mathrm{GHz}$.

o Can handle frequencies up to $110 \mathrm{GHz}$

- Receiving Antenna - SAS-571 Double Ridge Guide Horn Antenna

o Wideband antenna $(700 \mathrm{MHz}-18 \mathrm{GHz})$

o Approximately $12.2 \mathrm{dBi}$ of gain at $10 \mathrm{GHz}$

- SMA 3.5mm connector cable 


\subsubsection{Miscellaneous Equipment}

- Electric Power Generator

o Craftsman Model 580.329120

o $3000 \mathrm{~W}$

o AC $20 \mathrm{~A}$ and $120 \mathrm{~V}$

o DC $10 \mathrm{~A}$ and $12 \mathrm{~V}$

- Two $100 \mathrm{ft} .14$ gauge extension cords

- DC Power Supply

o Capable of generating $12 \mathrm{~V}$ and $6 \mathrm{~A}$

- Two fold-out tables to hold equipment

\subsubsection{Input Parameters and Predicted Performance}

As in all of the experiments, many different data parameters such as meteorological data and channel specifications were needed in order to accurately predict the received power. Site specific meteorological and locational data was provided by the Biology department and verified via the Google Earth Application. The altitude was determined through Google Earth.

The channel length, antenna heights, aperture dimensions, and cable losses were measured manually. Although losses due to multipath were expected, no additional losses were added to the model. The input parameters for the GUI at $10 \mathrm{GHz}$ is shown in Table 12. The predictive measurements during rain and in the clear-sky scenario can be seen in Table 13 and Table 14, respectively. 
Table 12. Input Parameters for a Link-Budget Calculation at $10 \mathrm{GHz}$

\begin{tabular}{|c|c|c|c|}
\hline \multicolumn{2}{|c|}{ Channel Specifications } & \multicolumn{2}{|c|}{ Transmitting System } \\
\hline Frequency & $10 \mathrm{GHz}$ & Antenna Gain & $12.2 \mathrm{dBi}$ \\
\hline Transmit Power & $25 \mathrm{dBm}$ & Largest Aperture Dimension & $0.24 \mathrm{~m}$ \\
\hline $\begin{array}{l}\text { Transmitter } \\
\text { Height }\end{array}$ & $5276 \mathrm{ft}$. & Antenna Efficiency & 0.5 \\
\hline Receiver Height & $5275.833 \mathrm{ft}$. & Pre-Amp Cable Losses & $8.0347 \mathrm{~dB}$ \\
\hline \multirow[t]{3}{*}{ Channel Length } & $.01788 \mathrm{~km}$ & Post- Amp Cable Losses & $3.2412 \mathrm{~dB}$ \\
\hline & & Amplifier Gain & $42 \mathrm{~dB}$ \\
\hline & & $\mathrm{P} 1$ & $40.3 \mathrm{dBm}$ \\
\hline \multicolumn{4}{|c|}{ Site Location } \\
\hline Longitude & 253.273749 & & \\
\hline Latitude & 34.344021 & \multicolumn{2}{|c|}{ Receiving System } \\
\hline \multicolumn{2}{|c|}{ Atmosphere } & Antenna Gain & $12.2 \mathrm{dBi}$ \\
\hline Temperature & $24.87 \mathrm{C}$ & Largest Aperture Dimension & $.24 \mathrm{~m}$ \\
\hline Pressure & $845.249 \mathrm{hPa}$ & Antenna Efficiency & 0.5 \\
\hline Humidity & $41.42 \%$ & Cable Losses & $2.0494 \mathrm{~dB}$ \\
\hline
\end{tabular}


Table 13. Link-Budget Calculation at $10 \mathrm{GHz}$ During a Rainfall at $20 \mathrm{~mm} / \mathrm{hour}$

\begin{tabular}{|c|c|c|c|c|c|}
\hline \multicolumn{6}{|c|}{ LINK BUDGET at $10 \mathrm{GHz}$ and $17.88 \mathrm{~m}$} \\
\hline Transmit Power & 25 & $\mathrm{dBm}$ & & & \\
\hline Transmit Antenna Gain & 12.2 & $\mathrm{dBi}$ & & & \\
\hline \multirow[t]{3}{*}{ EIRP } & 37.0588 & $\mathrm{dBm}$ & Free Space Path Loss & 77.4951 & $\mathrm{~dB}$ \\
\hline & & & Polarization Losses & 3.0103 & $\mathrm{~dB}$ \\
\hline & & & Gaseous Losses & 0.0001678 & $\mathrm{~dB}$ \\
\hline Receive Antenna Gain & 12.2 & $\mathrm{dBi}$ & Scintillation Losses & 0.0022 & $\mathrm{~dB}$ \\
\hline \multirow[t]{3}{*}{ Received Power } & -33.3796 & $\mathrm{dBm}$ & Rain Losses & 0.0834 & $\mathrm{~dB}$ \\
\hline & & & Clouds \& Fog Losses & 0 & $\mathrm{~dB}$ \\
\hline & & & Dust Losses & 0 & $\mathrm{~dB}$ \\
\hline System Noise Power & -177 & $\mathrm{dBW}$ & Misc. Losses & 0 & $\mathrm{~dB}$ \\
\hline $\mathrm{C} / \mathrm{N}$ & 143.6204 & $\mathrm{~dB}$ & Net Power Losses & 80.5890 & $\mathrm{~dB}$ \\
\hline C/N Threshold & -20 & $\mathrm{dBm}$ & & & \\
\hline Link Margin & 123.6240 & $\mathrm{~dB}$ & & & \\
\hline
\end{tabular}


Table 14. Link-Budget Calculation at $10 \mathrm{GHz}$ During Clear-Sky Atmosphere

\begin{tabular}{|c|c|c|c|c|c|}
\hline \multicolumn{6}{|c|}{ LINK BUDGET at $10 \mathrm{GHz}$ and $17.88 \mathrm{~m}$} \\
\hline Transmit Power & 25 & $\mathrm{dBm}$ & & & \\
\hline Transmit Antenna Gain & 12.2 & $\mathrm{dBi}$ & & & \\
\hline \multirow[t]{3}{*}{ EIRP } & 37.0588 & $\mathrm{dBm}$ & Free Space Path Loss & 77.4951 & $\mathrm{~dB}$ \\
\hline & & & Polarization Losses & 3.0103 & $\mathrm{~dB}$ \\
\hline & & & Gaseous Losses & 0.0001678 & $\mathrm{~dB}$ \\
\hline Receive Antenna Gain & 12.2 & $\mathrm{dBi}$ & Scintillation Losses & 0.0022 & $\mathrm{~dB}$ \\
\hline \multirow[t]{3}{*}{ Received Power } & -33.3796 & $\mathrm{dBm}$ & Rain Losses & 0 & $\mathrm{~dB}$ \\
\hline & & & Clouds \& Fog Losses & 0 & $\mathrm{~dB}$ \\
\hline & & & Dust Losses & 0 & $\mathrm{~dB}$ \\
\hline System Noise Power & -177 & $\mathrm{dBW}$ & Misc. Losses & 0 & $\mathrm{~dB}$ \\
\hline $\mathrm{C} / \mathrm{N}$ & 143.6204 & $\mathrm{~dB}$ & Net Power Losses & 80.5078 & $\mathrm{~dB}$ \\
\hline C/N Threshold & -20 & $\mathrm{dBm}$ & & & \\
\hline Link Margin & 123.6240 & $\mathrm{~dB}$ & & & \\
\hline
\end{tabular}

\subsection{Experiment 4-Quantifying Model Accuracy with Rain Impairment Again}

The fourth and final experiment was the culmination of the prior experiments. As mentioned previously, the original plan was to perform a couple of experiments with the 20 $\mathrm{mm} /$ hour rainfall, and one at $60 \mathrm{~mm} /$ hour. Following the first rain experiment, it was determined that the impact of precipitation on a $10 \mathrm{GHz}$ signal was minimal and could not be detected with a short range communication link. As a result, higher frequency operations were needed to analyze the model's accuracy. The specific attenuation at 10,20,30, and $40 \mathrm{GHz}$ was calculated, and it was determined that propagation at the higher frequencies would experience higher losses even with a small channel length.

Though it took some time, the $20-40 \mathrm{GHz}$ RF equipment that was used in the second experiment was made available to exploit in the final experiment. In order to perform the experiment with the equipment available, two channels needed to be created; one intended for 10 GHz propagation, and another that can perform 20,30, and $40 \mathrm{GHz}$ propagation. The high rain 
rates turned out to be very beneficial since they magnified the attenuation effect. Therefore, in order to maximize the impact that the rain would have on the wave propagation, the higher rainfall rate event was chosen for experimentation.

The goal of this final experiment was to measure the propagation losses across the plot at 10, 20, 30, and $40 \mathrm{GHz}$ prior to, during, and following the rain simulation. The hope was that noticeable losses would be measured and the results would match the link-budget model. As in the previous experiment, a generator that could support the equipment's power requirements was utilized. The experimental setup can be seen in Figure 22.

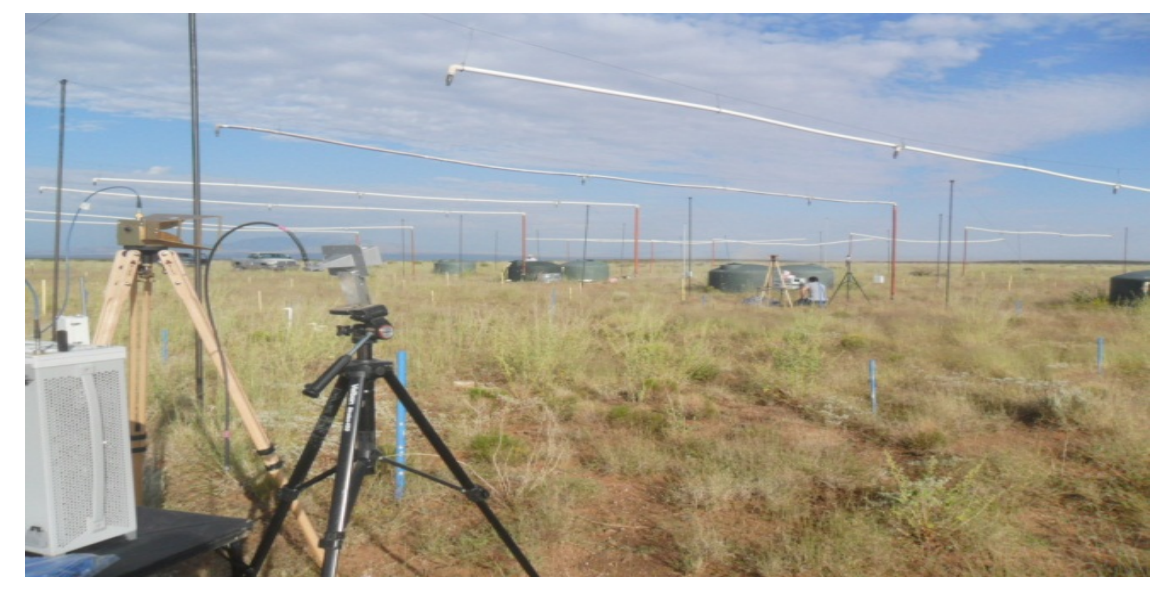

Figure 22. The Rainfall Experimental Setup; Note the Appreciable Height of the Water Distribution Lines and the Relatively Clear LOS Path

As mentioned previously, the Biologist group performed rain experiments throughout the summer. The original plans for the rain simulator experiment specified that measurements would be taken during several rain emulations to achieve a large amount of data. This was not the case though for two reasons:

- The original plan only included experiments at $10 \mathrm{GHz}$, and

- The equipment needed to perform this experiment at higher frequencies was only available for a small window of time.

As stated above, the plan originally specified several experiments at $10 \mathrm{GHz}$. The findings from the third experiment indicated that appreciable losses due to rain could not be accounted for precisely. The more sensitive frequencies were needed to be used and this will be discussed in 
more detail. Ultimately, the mission of this final experiment was to record measureable losses that could be cross-compared against the coupled model. The communication links operating at $10,20,30$, and $40 \mathrm{GHz}$ would hopefully show the rain loss relationship as expected.

As mentioned previously, two different communication links were created; one utilizing the original test equipment for $10 \mathrm{GHz}$, and another for the other three frequencies utilizing equipment rated for higher frequencies. The equipment used is split into five categories below; a transmitting system and receiving system for $10 \mathrm{GHz}$, a transmitting system and receiving system for 20,30, $40 \mathrm{GHz}$, and miscellaneous equipment. These are seen below.

\subsection{1 $10 \mathrm{GHz}$ Transmitting System}

- Signal Generator - HP 83752B Synthesized Sweeper

o Capable of generating continuous waves (CWs) with transmit power at 15 $\mathrm{dBm}$

o Frequency Range from $10 \mathrm{MHz}$ to $20 \mathrm{GHz}$

- Transmitting Antenna - SAS-571 Double Ridge Guide Horn Antenna

o Wideband antenna $(700 \mathrm{MHz}-18 \mathrm{GHz})$

o Approximately $12.2 \mathrm{dBi}$ of gain at $10 \mathrm{GHz}$

- X-Band Amplifier by Microsemi

o Provides approximately $42 \mathrm{~dB}$ of gain

o Has a saturation power at approximately $40.3 \mathrm{dBm}$

- 2 SMA $3.5 \mathrm{~mm}$ connector cables

\subsubsection{GHz Receiving System}

- Receiver - N9030A PXA Signal Analyzer

o Capable of measuring signal levels near $-159 \mathrm{dBm}$ at $10 \mathrm{GHz}$.

o Can handle frequencies up to $110 \mathrm{GHz}$

- Receiving Antenna - SAS-571 Double Ridge Guide Horn Antenna

o Wideband antenna $(700 \mathrm{MHz}-18 \mathrm{GHz})$

o Approximately $12.2 \mathrm{dBi}$ of gain at $10 \mathrm{GHz}$

- SMA 3.5mm connector cable 


\subsubsection{0, 30, and 40 GHz Transmitting System}

- Signal Generator - Agilent E8257D

o Capable of generating $\mathrm{CW}$ with transmit power at $15 \mathrm{dBm}$

o Maximum Operating Frequency - $50 \mathrm{GHz}$

- Transmitting Antenna - AH-840 Horn Antenna

o Broadband antenna $(18-40 \mathrm{GHz})$

o Approximately 21.63, 23.65, and $21.05 \mathrm{dBi}$ of gain at 20,30 , and $40 \mathrm{GHz}$ respectively

\subsubsection{0, 30, and 40 GHz Receiving System}

- Receiver - N9030A PXA Signal Analyzer

o Capable of measuring signal levels near $-159 \mathrm{dBm}$ at $10 \mathrm{GHz}$.

o Can handle frequencies up to $110 \mathrm{GHz}$

- Receiving Antenna - AH-840 Horn Antenna

o Broadband antenna $(18-40 \mathrm{GHz})$

o Approximately $21.63,23.65$, and $21.05 \mathrm{dBi}$ of gain at 20,30 , and $40 \mathrm{GHz}$, respectively.

\subsubsection{Miscellaneous Equipment}

- Electric Power Generator

o Craftsman Model 580.329120

o $3000 \mathrm{~W}$

o AC $20 \mathrm{~A}$ and $120 \mathrm{~V}$

O DC $10 \mathrm{~A}$ and $12 \mathrm{~V}$

- Two $100 \mathrm{ft} .14$ gauge extension cords

- DC Power Supply

o Capable of generating $12 \mathrm{~V}$ and $6 \mathrm{~A}$

- Two $6 \mathrm{ft}$. X $8 \mathrm{ft}$. tarps (for equipment protection)

- Two fold-out tables to hold equipment. 


\subsubsection{Input Parameters and Predicted Performance}

Many different data parameters such as meteorological data and channel specifications were needed in order to accurately predict the received power. Site specific meteorological data was utilized to achieve maximum accuracy for modeling. The receiver location was provided by the Biology department and verified via the Google Earth Application. The altitude was determined through Google Earth. The channel length was measured across the measurement area. The antenna heights and aperture dimensions were measured by hand. The cable losses were measured with a network analyzer following experimentation. Although losses due to multipath were expected, no additional losses were added to the model. The input data for the GUI at $10 \mathrm{GHz}$ and $20-40 \mathrm{GHz}$ are shown in Table 15 and Table 16, respectively.

Table 15. Input Parameters for a Link-Budget Calculation at $10 \mathrm{GHz}$

\begin{tabular}{|l|r|l|r|}
\hline \multicolumn{2}{|c|}{ Channel Specifications } & \multicolumn{2}{c|}{ Transmitting System } \\
\hline Frequency & $10 \mathrm{GHz}$ & Antenna Gain & $12.2 \mathrm{dBi}$ \\
\hline Transmit Power & $25 \mathrm{dBm}$ & Largest Aperture Dimension & $0.24 \mathrm{~m}$ \\
\hline $\begin{array}{l}\text { Transmitter } \\
\text { Height }\end{array}$ & $5276.25 \mathrm{ft}$. & Antenna Efficiency & 0.5 \\
\hline Receiver Height & $5276.125 \mathrm{ft}$. & Pre-Amp Cable Losses & $1.63 \mathrm{~dB}$ \\
\hline Channel Length & $0.0181356 \mathrm{~km}$ & Post- Amp Cable Losses & $7.10 \mathrm{~dB}$ \\
\hline & & Amplifier Gain & $42 \mathrm{~dB}$ \\
\hline \multicolumn{2}{|c|}{ Site Location } & P1 & $40.3 \mathrm{dBm}$ \\
\hline \multicolumn{2}{|c|}{ Atmosphere } & Antenna Gain & \\
\hline Longitude & 253.273749 & \multicolumn{2}{c|}{ Receiving System } \\
\hline Latitude & $26.44 \mathrm{C}$ & Largest Aperture Dimension & $0.24 \mathrm{~m}$ \\
\hline \multicolumn{2}{|r|}{$845.514 \mathrm{hPa}$} & Antenna Efficiency & 0.5 \\
\hline Temperature & $43.37 \%$ & Cable Losses & $2.36 \mathrm{~dB}$ \\
\hline Pressure & & & $12.2 \mathrm{dBi}$ \\
\hline Humidity & & &
\end{tabular}


Table 16. Input Parameters for a Link-Budget Calculation at 20, 30, and $40 \mathrm{GHz}$

\begin{tabular}{|c|c|c|c|}
\hline \multicolumn{2}{|c|}{ Channel Specifications } & \multicolumn{2}{|c|}{ Transmitting System } \\
\hline \multirow{2}{*}{ Frequency } & \multirow{2}{*}{$\begin{array}{r}20,30,40 \\
\mathrm{GHz}\end{array}$} & Antenna Gain & \multirow{2}{*}{$\begin{array}{r}21.63,23.65 \\
21.05 \mathrm{~dB}\end{array}$} \\
\hline & & $(20,30,40 \mathrm{GHz})$ & \\
\hline Transmit Power & $15 \mathrm{dBm}$ & $\begin{array}{l}\text { Largest Aperture } \\
\text { Dimension }\end{array}$ & $0.06858 \mathrm{~m}$ \\
\hline Transmitter Height & $5276.6667 \mathrm{ft}$. & Antenna Efficiency & 0.5 \\
\hline \multirow{2}{*}{ Receiver Height } & \multirow{2}{*}{$5276.5 \mathrm{ft}$. } & Cable Losses & \multirow{2}{*}{$3.39,4.33,7.32$} \\
\hline & & $(20,30,40 \mathrm{GHz})$ & \\
\hline Channel Length & $0.0172 \mathrm{~km}$ & & \\
\hline \multicolumn{2}{|c|}{ Site Location } & & \\
\hline Longitude & 253.273749 & & \\
\hline Latitude & 34.344021 & \multicolumn{2}{|c|}{ Receiving System } \\
\hline \multicolumn{2}{|c|}{ Atmosphere } & Antenna Gain & $12.2 \mathrm{dBi}$ \\
\hline Temperature & $26.44 \mathrm{C}$ & $\begin{array}{l}\text { Largest Aperture } \\
\text { Dimension }\end{array}$ & $0.06858 \mathrm{~m}$ \\
\hline Pressure & $845.514 \mathrm{hPa}$ & Antenna Efficiency & 0.5 \\
\hline \multirow[t]{2}{*}{ Humidity } & $43.27 \%$ & Cable Losses & $\begin{array}{r}2.36,2.83,4.86 \\
\mathrm{~dB}\end{array}$ \\
\hline & & $(20,30,40 \mathrm{GHz})$ & \\
\hline
\end{tabular}

As in the previous experiments, the channels were evaluated using the coupled model using the given input parameters. In this case, there was a total of eight simulations ran. To make the reading more convenient, only the received power and expected rain losses will be included in Table 17. Note that the frequencies in Table 17 cannot be directly compared to each other due to the different antenna gains, cables losses, and completely different transmitting systems as in the case of the $10 \mathrm{GHz}$ channel. 
Table 17. Expected Performance at 10, 20, 30, and $40 \mathrm{GHz}$

\begin{tabular}{|c|c|c|c|}
\hline $\begin{array}{c}\text { Frequency } \\
(\mathbf{G H z})\end{array}$ & $\begin{array}{c}\text { Clear-Sky Received } \\
\text { Power (dBm) }\end{array}$ & $\begin{array}{c}\text { Received Power } \\
\text { with Rain } \mathbf{( d B m )}\end{array}$ & Rain Losses (dB) \\
\hline 10 & -37.59 & -37.84 & 0.25 \\
\hline 20 & -33.69 & -34.65 & 0.97 \\
\hline 30 & -34.58 & -36.51 & 1.94 \\
\hline 40 & -47.30 & -50.28 & 2.99 \\
\hline
\end{tabular}

\section{RESULTS AND DISCUSSION}

\subsection{Experiment 1 - Measuring Propagation Losses}

In this first experiment, no redundant measurements were taken at any power. The raw power measured at $10 \mathrm{GHz}$ with their respective input power levels are conveyed in Table 18 and Figure 23. The raw power readings are seen in Figure 24 through Figure 28. From the figures, it is evident that the frequency spanned from 9 to $11 \mathrm{GHz}$. This allowed for non-typical signals to be detected in the received power measurements. From these figures, the center frequency is easily spotted, even at the low transmission power of $14 \mathrm{dBm}$ as seen in Figure 28.

Table 18. Measured Received Power at $10 \mathrm{GHz}$ for a Channel Length of $46 \mathrm{~m}$.

\begin{tabular}{|c|c|}
\hline TX Power (dBm) & RX Power $(\mathbf{d B m})$ \\
\hline 11 & -58.64 \\
\hline 14 & -57.46 \\
\hline 17 & -54.67 \\
\hline 20 & -52.12 \\
\hline 23 & -50.94 \\
\hline
\end{tabular}




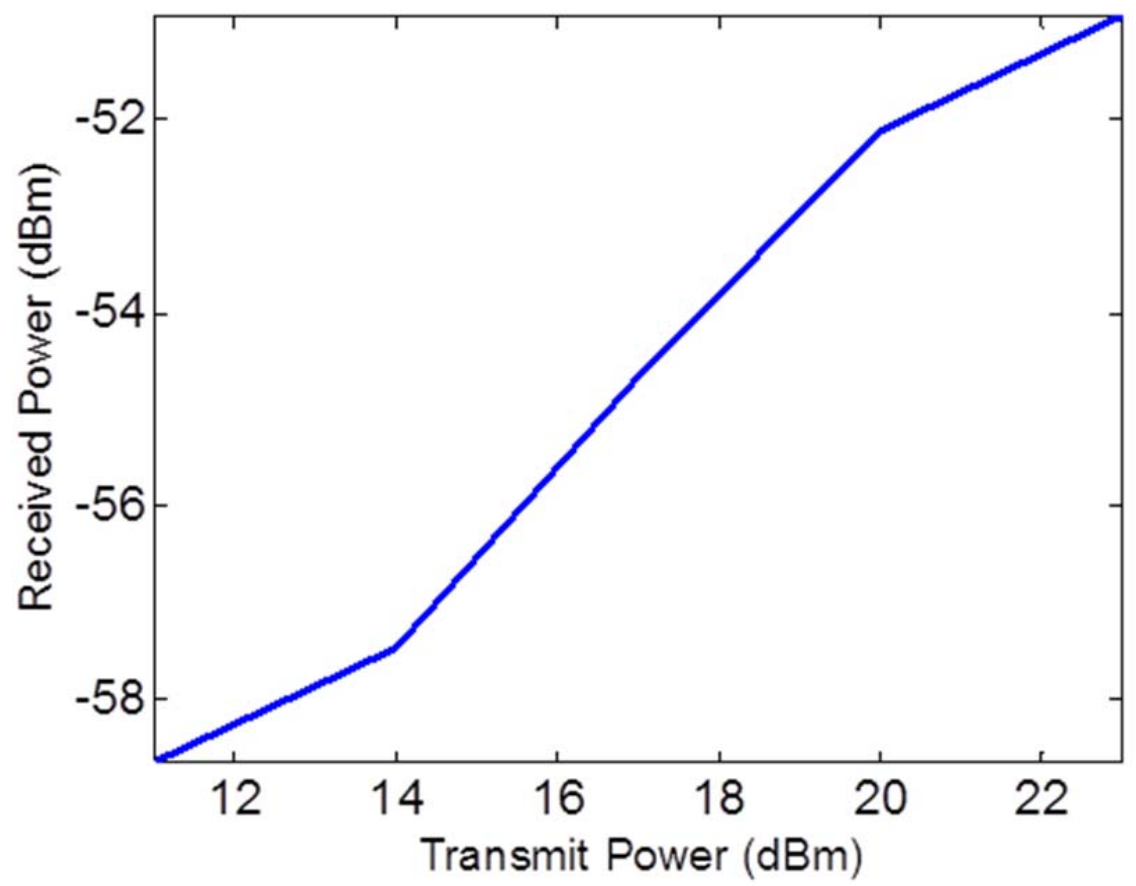

Figure 23. Measured Received Power at $10 \mathrm{GHz}$ for a Channel Length of $46 \mathrm{~m}$

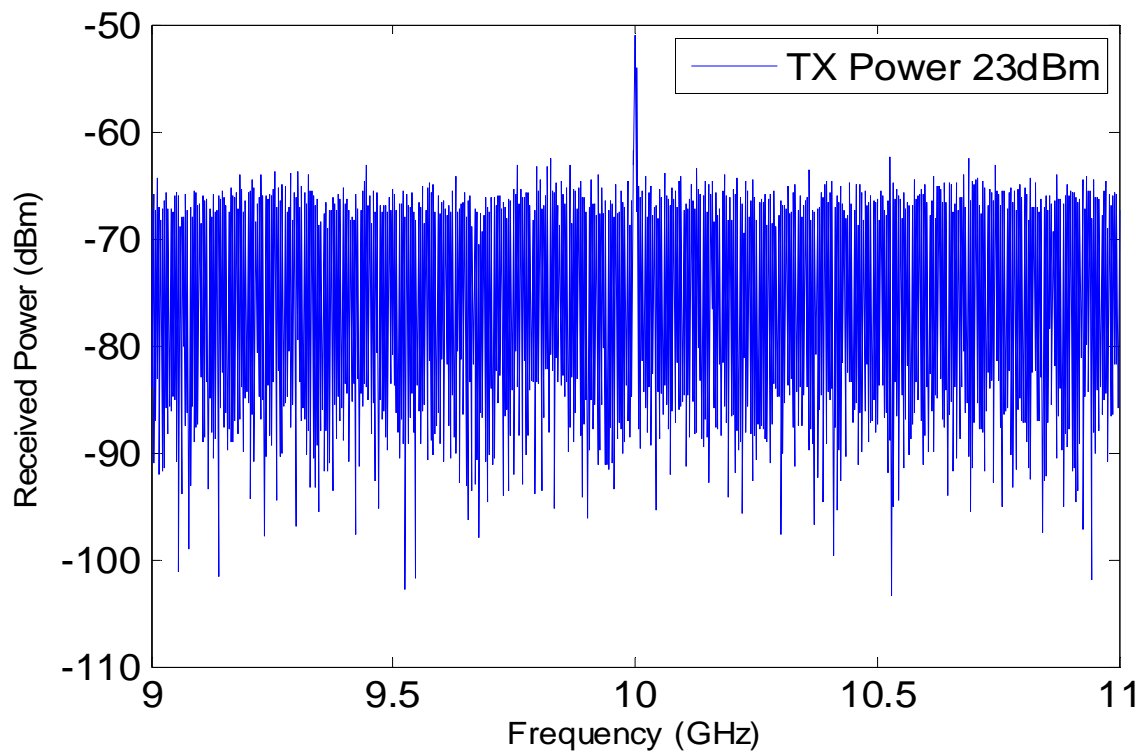

Figure 24. Measured Received Power at $10 \mathrm{GHz}$ for a Transmit Power of $23 \mathrm{dBm}$ 


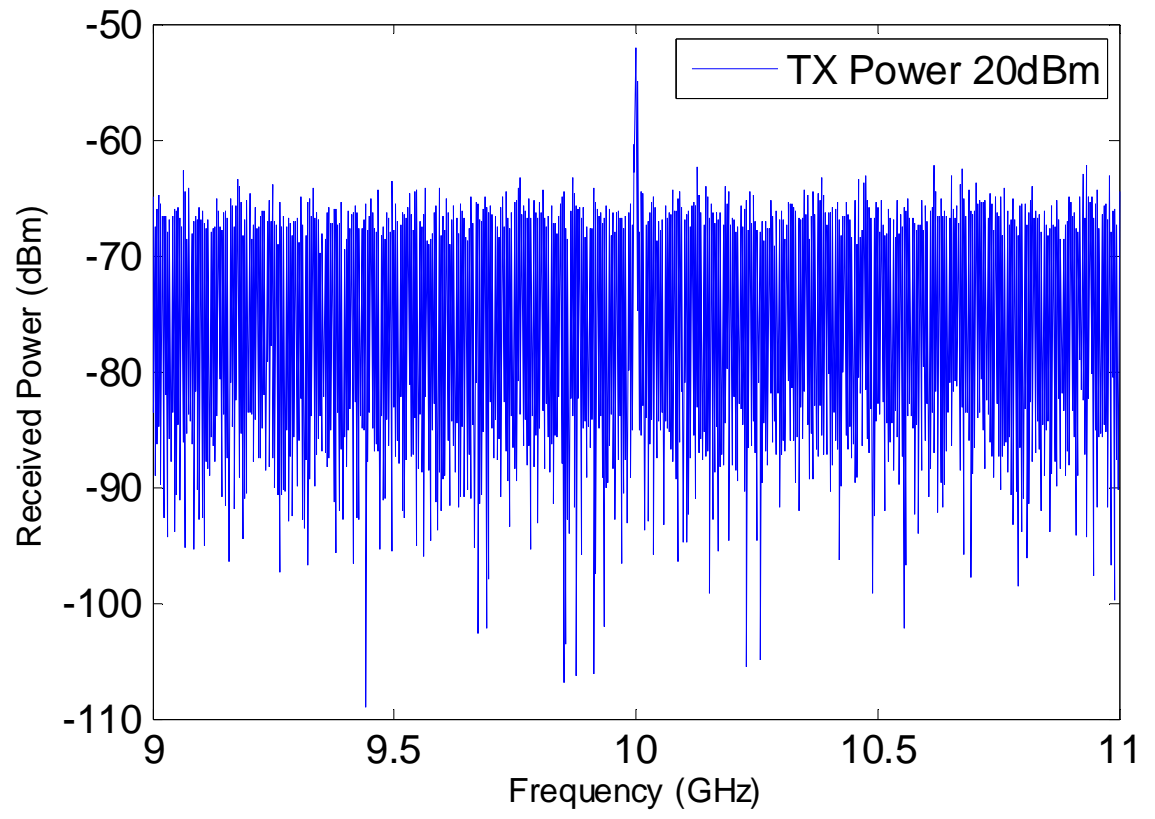

Figure 25. Measured Received Power at $10 \mathrm{GHz}$ for a Transmit Power of $20 \mathrm{dBm}$

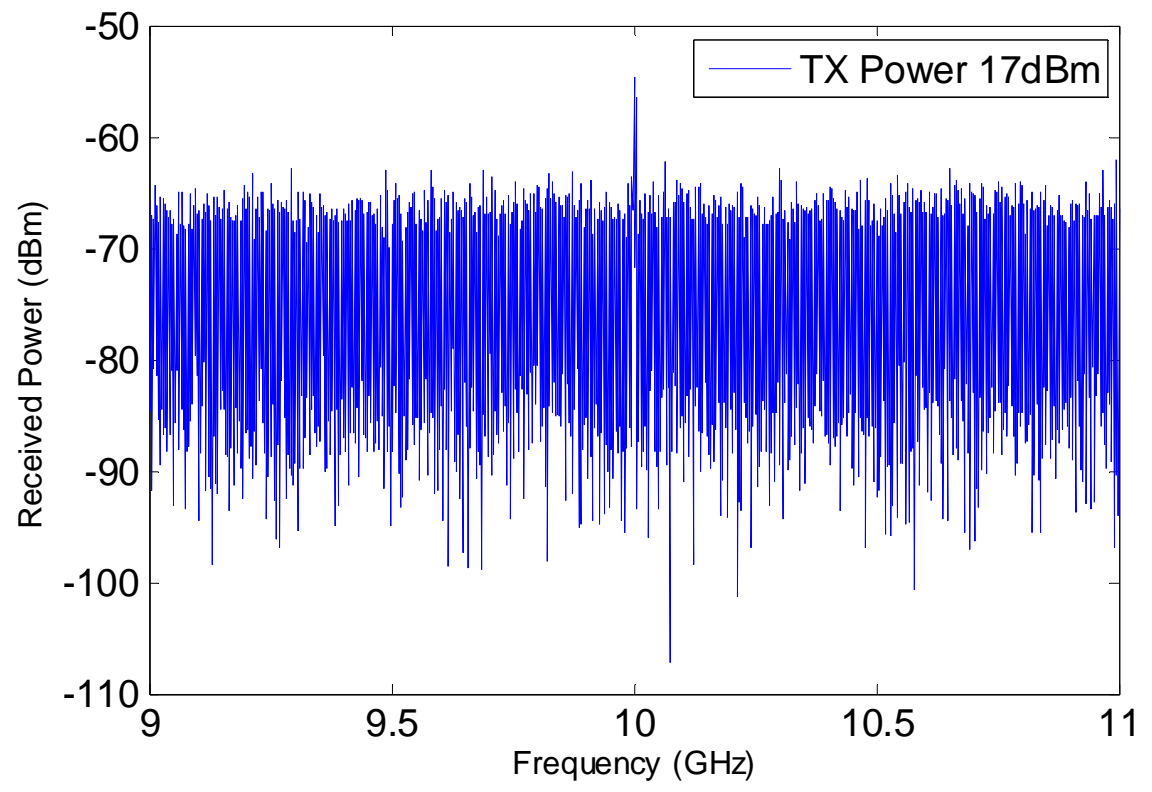

Figure 26. Measured Received Power at $10 \mathrm{GHz}$ for a Transmit Power of $17 \mathrm{dBm}$ 


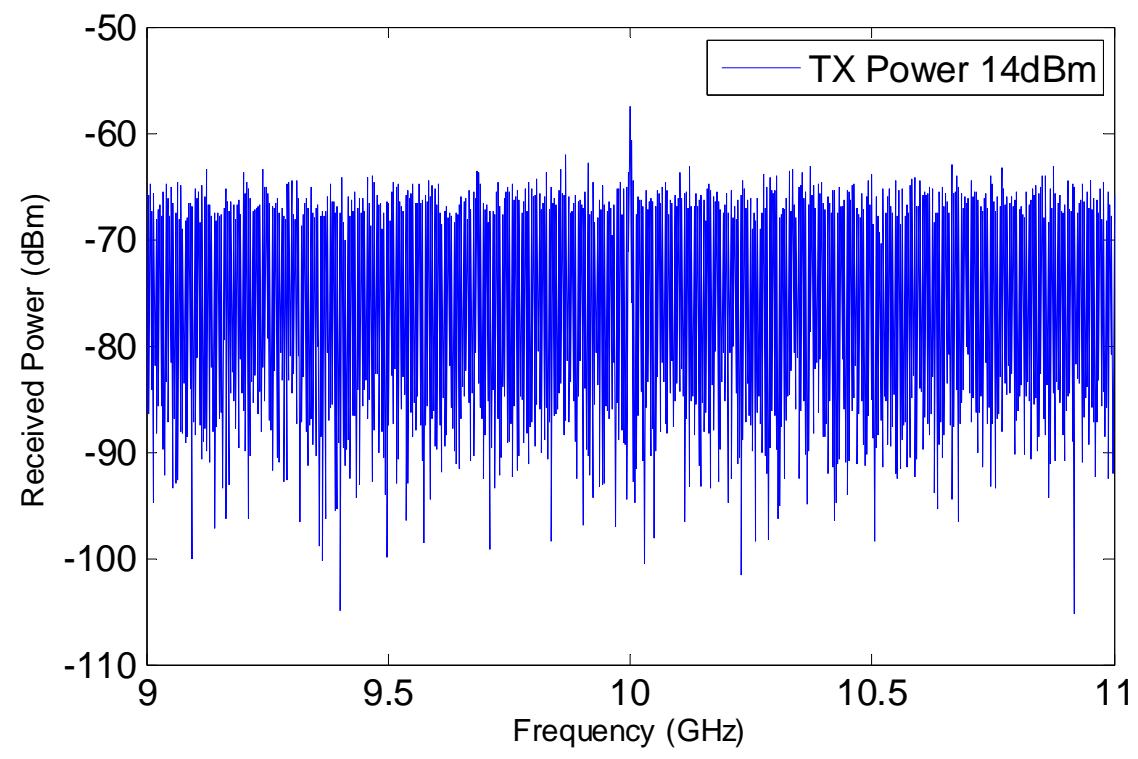

Figure 27. Measured Received Power at $10 \mathrm{GHz}$ for a Transmit Power of $14 \mathrm{dBm}$

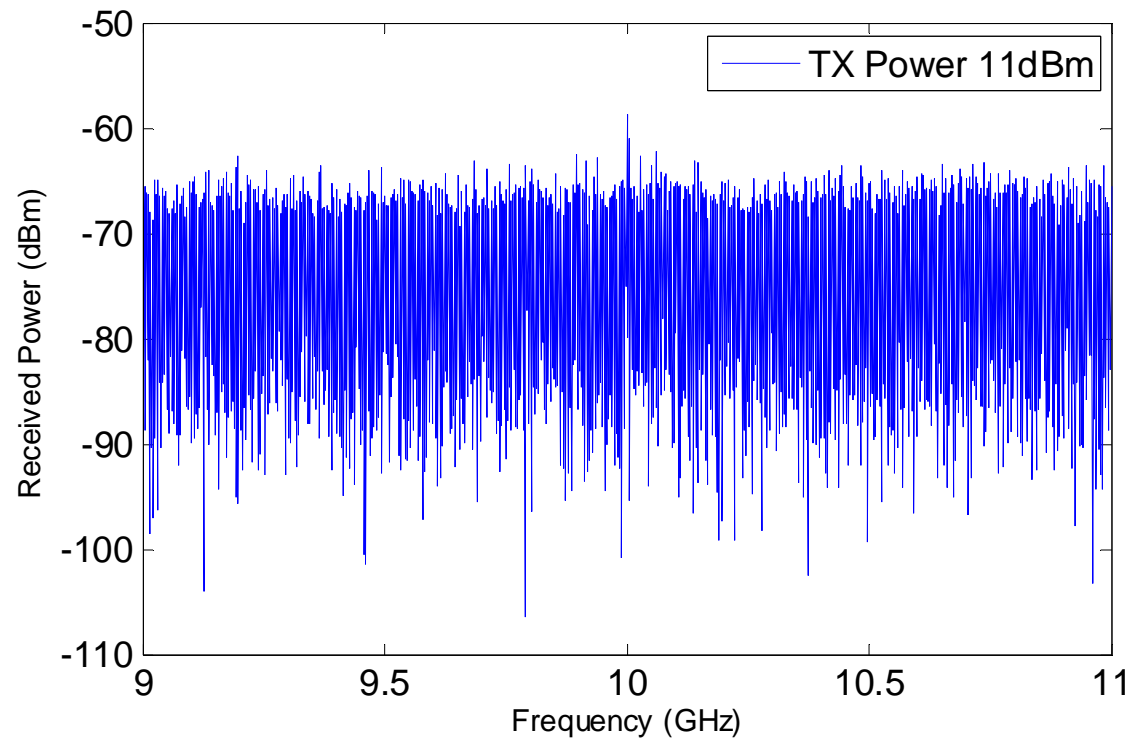

Figure 28. Measured Received Power at $10 \mathrm{GHz}$ for a Transmit Power of $11 \mathrm{dBm}$

A link-budget calculation was performed for this experiment only using $23 \mathrm{dBm}$ as the transmission power. In the actual experiment, this was not the case. Received power levels were measured for five different transmission powers; 11, 14, 17, 20, and $23 \mathrm{dBm}$. Following retrieval 
of the data captured, the measured values were compared to the predicted model values, which is conveyed in Figure 29 and displayed in Table 19.

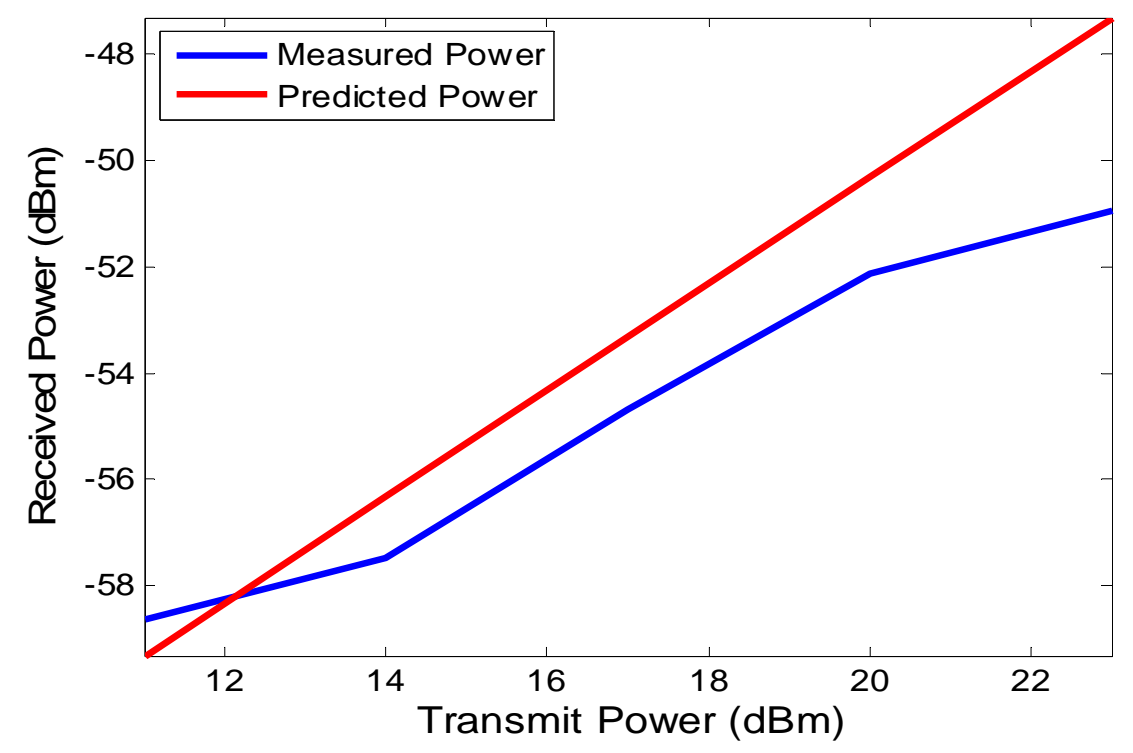

Figure 29. The Predicted Received Power Compared Against the Measured Values

Table 19. The Initial Comparison Between the Measured Results and the Predicted Data

\begin{tabular}{|c|c|c|c|}
\hline $\begin{array}{c}\text { Transmitted } \\
\text { Power (dB) }\end{array}$ & $\begin{array}{c}\text { Predicted } \\
\text { Receive } \\
\text { Power (dB) }\end{array}$ & $\begin{array}{c}\text { Measured } \\
\text { (dB) }\end{array}$ & $\begin{array}{c}\text { Difference } \\
\text { (dB) }\end{array}$ \\
\hline 11 & -59.31 & -58.64 & 0.67 \\
\hline 14 & -56.31 & -57.46 & -1.15 \\
\hline 17 & -53.31 & -54.67 & -1.36 \\
\hline 20 & -50.31 & -52.12 & -1.81 \\
\hline 23 & -47.31 & -50.94 & -3.63 \\
\hline
\end{tabular}

From Table 19, two simple observations can be made, (1) the received power was measured successfully, and (2) the prediction model is relatively close to the measured power. At this point, the initial goals of the first experiment were met. However, it was necessary to analyze the data received in order to improve the current model, and understand any 
shortcomings. In Figure 29, the predicted power follows a linear relationship, whereas the measured data moves around. The predicted power also appears to be higher than that of the measured values for most transmitted powers. The measured power and predicted powers should theoretically match exactly if performed correctly.

In theory, the power measured should follow a linear relationship, as long as the input power does not surpass the antenna limitations. From Figure 29, the power appears to be rolling off, as if it were somehow saturating. The SAS 571 horn antenna is specified to have a maximum continuous power of $300 \mathrm{~W}$, which is approximately $54.8 \mathrm{dBm}$. The input powers for this experiment clearly do not pass this threshold with the largest power being $23 \mathrm{dBm}$ without additional losses. Since no violation is present, the error must come from the measuring technique, unaccounted losses, or a combination of the two. This is a much more probable explanation given the propagation environment and the lack of experience in recording measurements (at the time).

In Figure 19, the environmental propagation impairments can be observed. Notice that the roof walls are not much lower than the antennas' heights. This could be problematic for transmitted signal with low directivity and conversely large beamwidth. Although it was not considered at the time of experimentation, the E-Field and H-Field half-power beam-widths for the SAS 571 antenna are specified to be $48^{\circ}$ and $30^{\circ}$, respectively. In this experiment, the electric field was positioned in parallel with ground, which makes the magnetic field perpendicular. Since the beamwidth for the $\mathrm{H}$-field is $30^{\circ}$, some interaction between the roof wall and the signal could be expected.

Another loss mechanism not accounted for is the pointing loss. In the experiment, an attempt to mitigate this loss as much as possible was made. The antennas were moved individually on both sides to maximize the received power seen on the spectrum analyzer. It was believed that the antennas were positioned in the optimum spot, though small losses could still exist.

The possible explanation for the varying power losses could be that there was a significant lack of data to be modeled linearly. As mentioned previously, only one measurement at each power was taken, which definitely supports this theory. In an attempt to quantify the accuracy, the measured and respective powers were normalized to their effective isotropic radiated power (EIRP), and then to the average. From this, an estimation of how well the model 
performed, in general, can be found. The data supporting this is listed in Table 20 and can be seen in Figure 30. The power measured and predicted at the antenna, rather than the source, was found by simply removing the cable losses from the received powers.

Although this statistic states nothing about the model performance with varying powers, it does convey the accuracy of the model with only two outliers being present in Figure 30. Even with five data points, there appears to be no clear convergence in the measured power. This makes it difficult to clearly quantify the accuracy. The only conclusion that can be made in this case is that the majority of data points (3/5) are within the error bars.

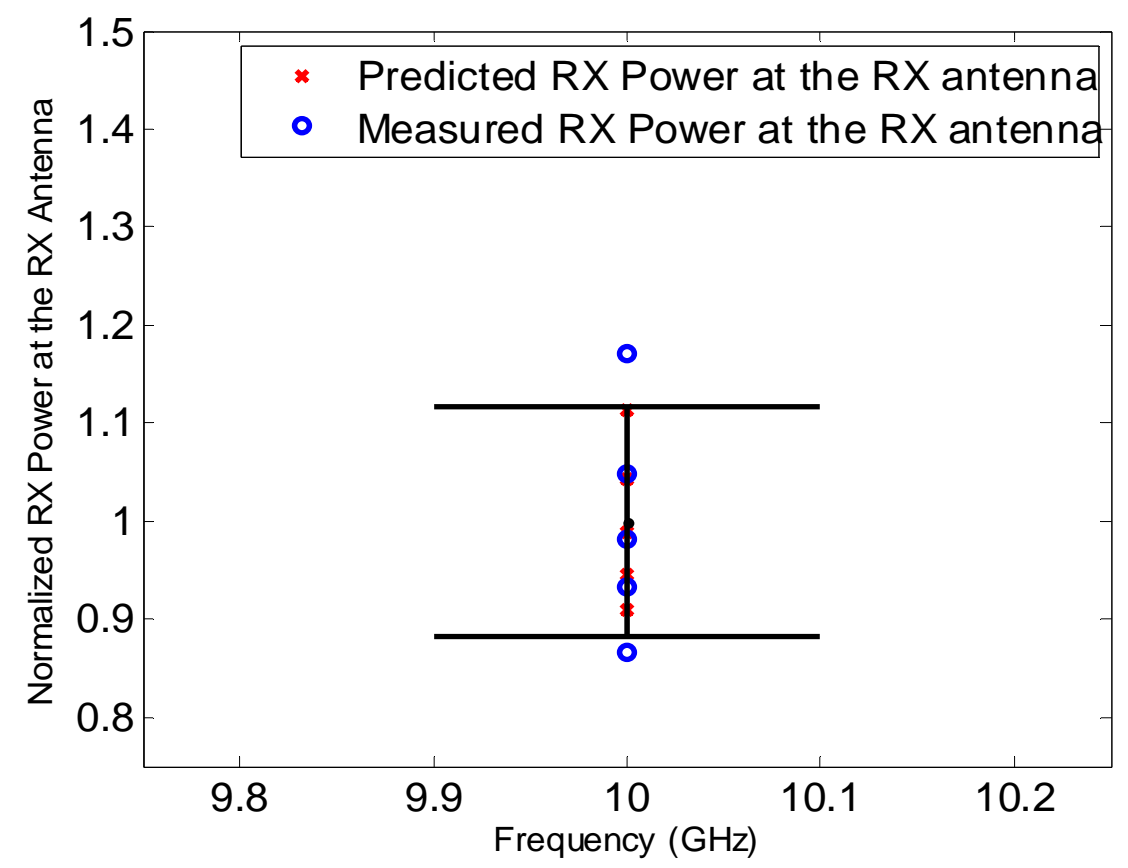

Figure 30. Data Comparison Between the Normalized Data of the Predicted and Measured Powers 
Table 20. The Initial Comparison Between the Measured Results and the Predicted Data

\begin{tabular}{|c|c|c|c|c|c|}
\hline $\begin{array}{c}\text { Predicted Receive Power at } \\
\text { Antenna (dB) }\end{array}$ & $\mathbf{- 5 1 . 6 0}$ & $\mathbf{- 4 8 . 6 0}$ & $\mathbf{- 4 5 . 6 0}$ & $\mathbf{- 4 2 . 6 0}$ & $\mathbf{- 3 9 . 6 0}$ \\
\hline $\begin{array}{c}\text { Measured Receive Power at } \\
\text { Antenna (dB) }\end{array}$ & -50.93 & -49.75 & -46.96 & -44.41 & -43.23 \\
\hline EIRP (dB) & 32.2 & 29.2 & 26.2 & 23.2 & 20.2 \\
\hline Predicted/ EIRP & -1.603 & -1.66 & -1.74 & -1.84 & -1.96 \\
\hline Measured/ EIRP & -1.58 & -1.70 & -1.79 & -1.91 & -2.14 \\
\hline (Predicted/EIRP)/AVG & 0.91 & 0.95 & 0.99 & 1.04 & 1.11 \\
\hline (Measured/EIRP)/AVG & 0.87 & 0.93 & 0.98 & 1.05 & 1.17 \\
\hline
\end{tabular}

\subsection{Experiment 2 - Quantifying Model Accuracy in an Anechoic Chamber}

The primary goal was to quantify the accuracy of the link-budget model at $10 \mathrm{GHz}$ in this experiment. The secondary goal was to vary frequency upwards to determine if the model is scalable.

\subsubsection{Measurements}

For reference, the input parameters for the $10 \mathrm{GHz}$ and 20, 30, and $40 \mathrm{GHz}$ link-budget calculations can be seen in Table 21. The measured received power for each link can be seen in Figure 31 through Figure 34. Red lines indicate "error bars”. The data measured at each carrier frequency is displayed in Table 21. In each of the Figure 31 through Figure 34, it is clear where the signal lies. Since all signals were detected properly, analysis can be performed. In Table 21, it can be seen that a total of eight measurements were taken at each of the frequencies of interest, where the number of measurements is arbitrary. Prior to the analysis, note that $10 \mathrm{GHz}$ was the main focus at the time. 
Table 21. The Measured Power Values at the Primary Frequencies of Interest

\begin{tabular}{|c|c|c|c|c|}
\hline $\begin{array}{c}\text { Frequency } \\
\text { (GHz) }\end{array}$ & $\mathbf{1 E + 1 0}$ & $\mathbf{2 E + 1 0}$ & $\mathbf{3 E + 1 0}$ & $\mathbf{4 E + 1 0}$ \\
\hline Meas. 1 & -42.7814 & -34.5725 & -34.5748 & -44.9946 \\
\hline Meas. 2 & -42.8248 & -34.5313 & -34.7717 & -44.0781 \\
\hline Meas. 3 & -42.8887 & -34.6085 & -34.8136 & -45.5619 \\
\hline Meas. 4 & -43.0526 & -34.499 & -35.0304 & -44.8704 \\
\hline Meas. 5 & -43.1106 & -34.7761 & -34.7129 & -45.3473 \\
\hline Meas. 6 & -43.0526 & -34.6342 & -34.793 & -44.0957 \\
\hline Meas. 7 & -43.1364 & -34.6555 & -34.8739 & -44.8976 \\
\hline Meas. 8 & -43.0702 & -34.5637 & -34.6798 & -43.9252 \\
\hline Average & -42.9897 & -34.6051 & -34.7813 & -44.7214 \\
\hline
\end{tabular}

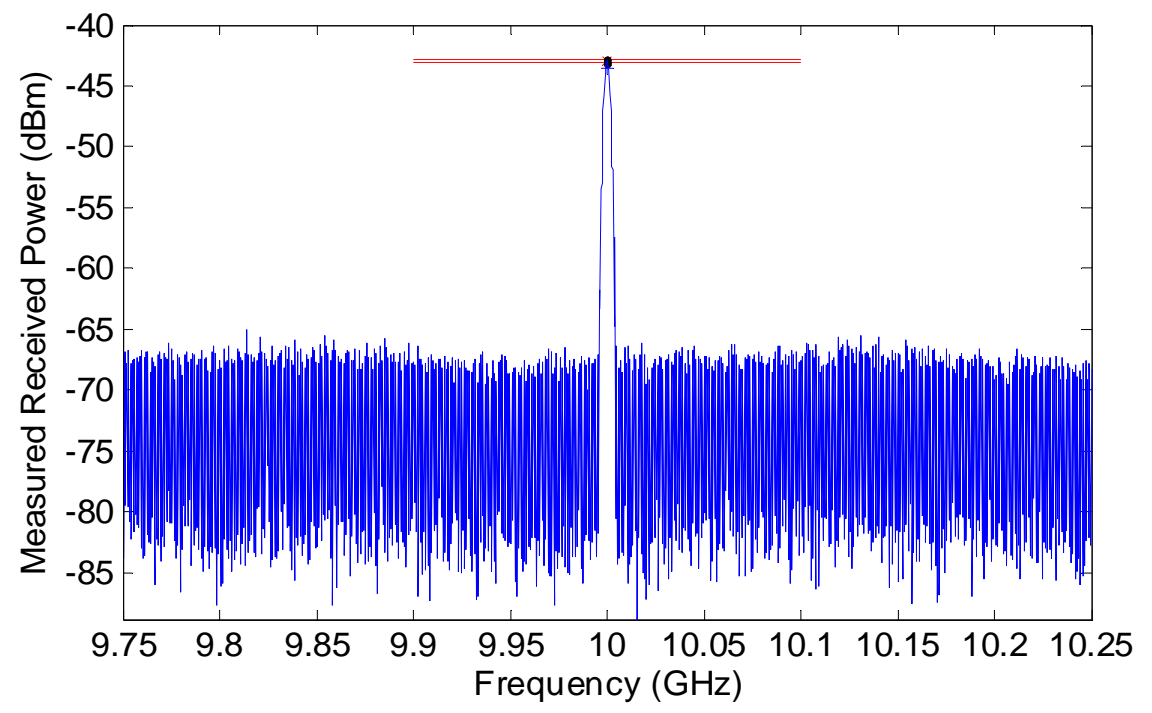

Figure 31. Plot of the Raw Output Data with Maximum Power Received at $10 \mathrm{GHz}$ 


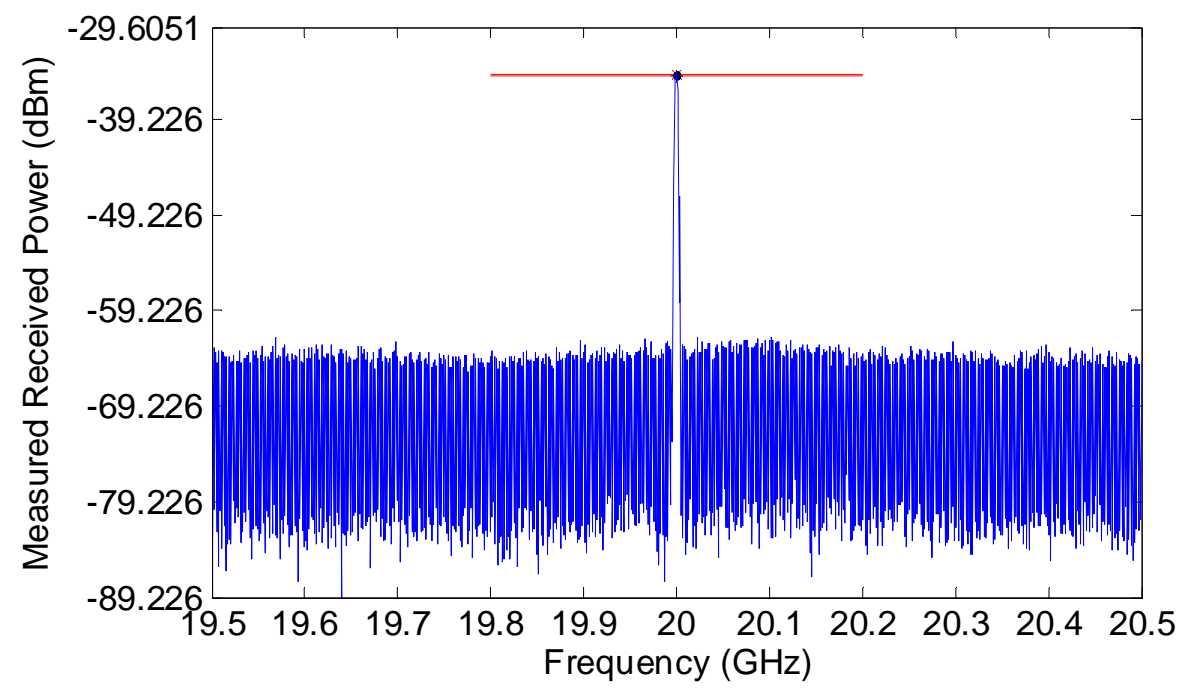

Figure 32. Plot of the Raw Output Data with Maximum Power Received at 20 GHz

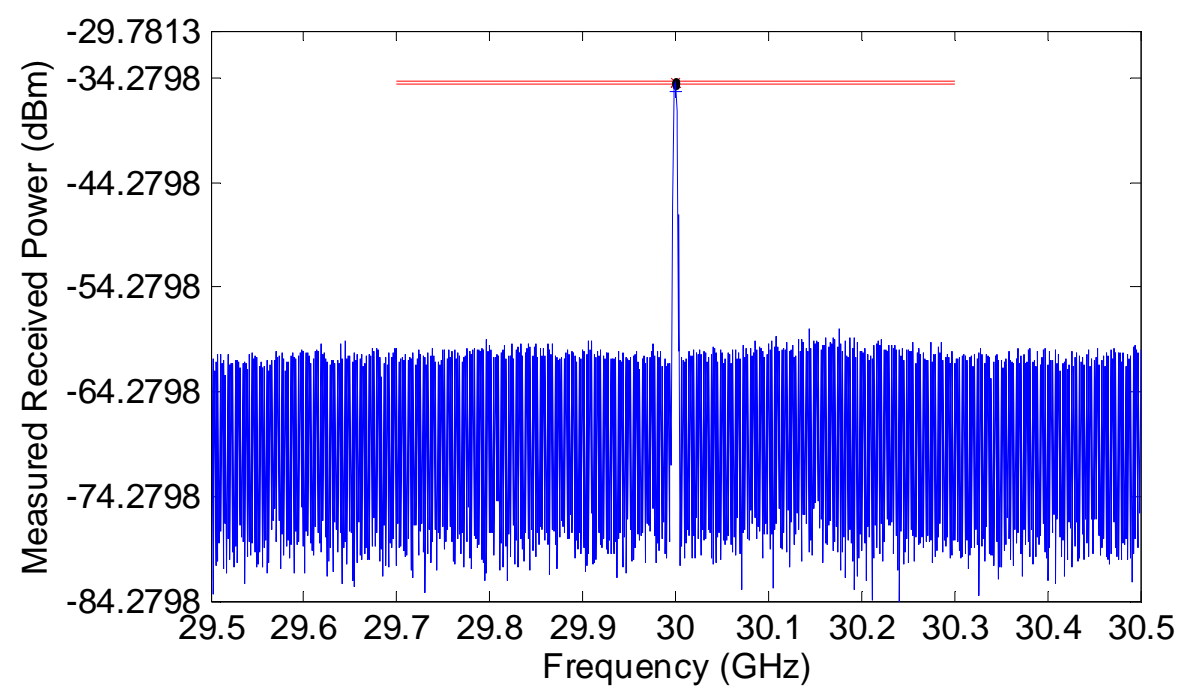

Figure 33. Plot of the Raw Output Data with Maximum Power Received at $30 \mathrm{GHz}$ 


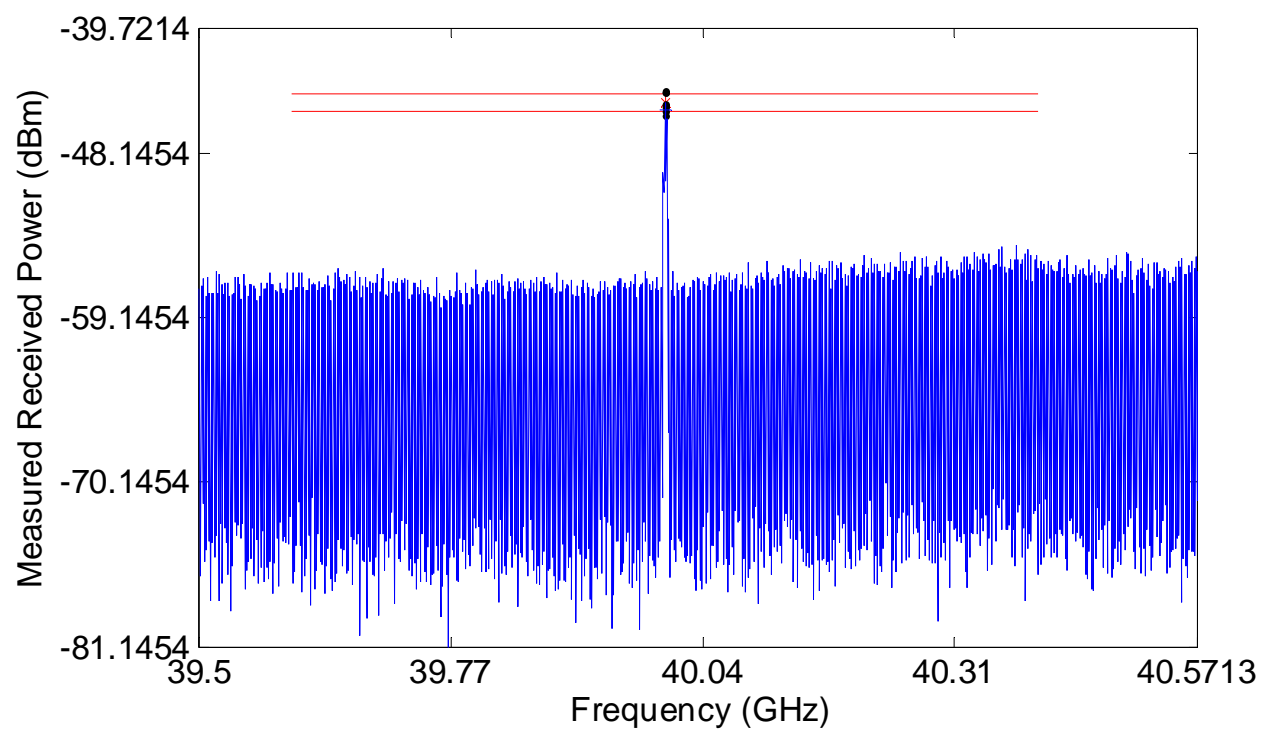

Figure 34. Plot of the Raw Output Data With Maximum Power Received at 40 GHz

\subsubsection{Analysis}

In Figure 21, the raw data measurements for the frequencies of interest can be seen for this experiment. It was desired to determine how accurate the model was in comparison to the ideal case. Therefore the first comparison that was performed was an absolute difference calculation. This can be seen in Figure 22.

Table 22. Measured Power Values at the Primary Frequencies of Interest

\begin{tabular}{|c|c|c|c|c|}
\hline Frequency & $\mathbf{1 0 ~ G H z}$ & $\mathbf{2 0 ~ G H z}$ & $\mathbf{3 0 ~ G H z}$ & $\mathbf{4 0} \mathbf{~ G H z}$ \\
\hline $\begin{array}{c}\text { Predicted Power } \\
\mathbf{( d B m )}\end{array}$ & -43.57 & -34.73 & -35.64 & -45.18 \\
\hline $\begin{array}{c}\text { Average Measured } \\
\text { Power (dBm) }\end{array}$ & -42.99 & -34.60 & -34.78 & -44.72 \\
\hline $\begin{array}{c}\text { Absolute Difference } \\
\mathbf{( d B m )}\end{array}$ & 0.58 & 0.13 & 0.85 & 0.45 \\
\hline
\end{tabular}


The difference appears to be minimal with the largest deviation being $0.85 \mathrm{~dB}$ at $30 \mathrm{GHz}$. However, it was believed that the model should nearly mimic the measured values with the only unaccounted loss being the pointing loss. To truly examine how well the predicted power compares to the measured values, it is necessary to take a closer look at the error bar regions in the Figure 31 through Figure 34. The zoomed in images can be seen in Figure 35 through Figure 38. In these plots, an error bar is present which was calculated utilizing the average of the measured powers associated with each frequency. The standard deviations for 10, 20, 30, and 40 GHz signal measurements are $0.128,0.081,0.127$, and 0.577 , respectively. From these values, the spread of the measurements can be seen. Notice that the $40 \mathrm{GHz}$ plot has the largest spread and that can be clearly conveyed in Figure 38. Along with the error bars, the predicted value and measured powers are also plotted on the figure represented by a blue cross and black dots respectively. With the majority of data points within the error bars, the number of measurements is believed to be sufficient. In Figure 35, notice that the predicted power is about $0.6 \mathrm{dBm}$ lower than the measured powers. This is slightly troublesome, since it was expected that in an ideal scenario the model should be in the same region as the measurements. Though, one could argue that a model that over anticipates losses is better than one that underestimates.

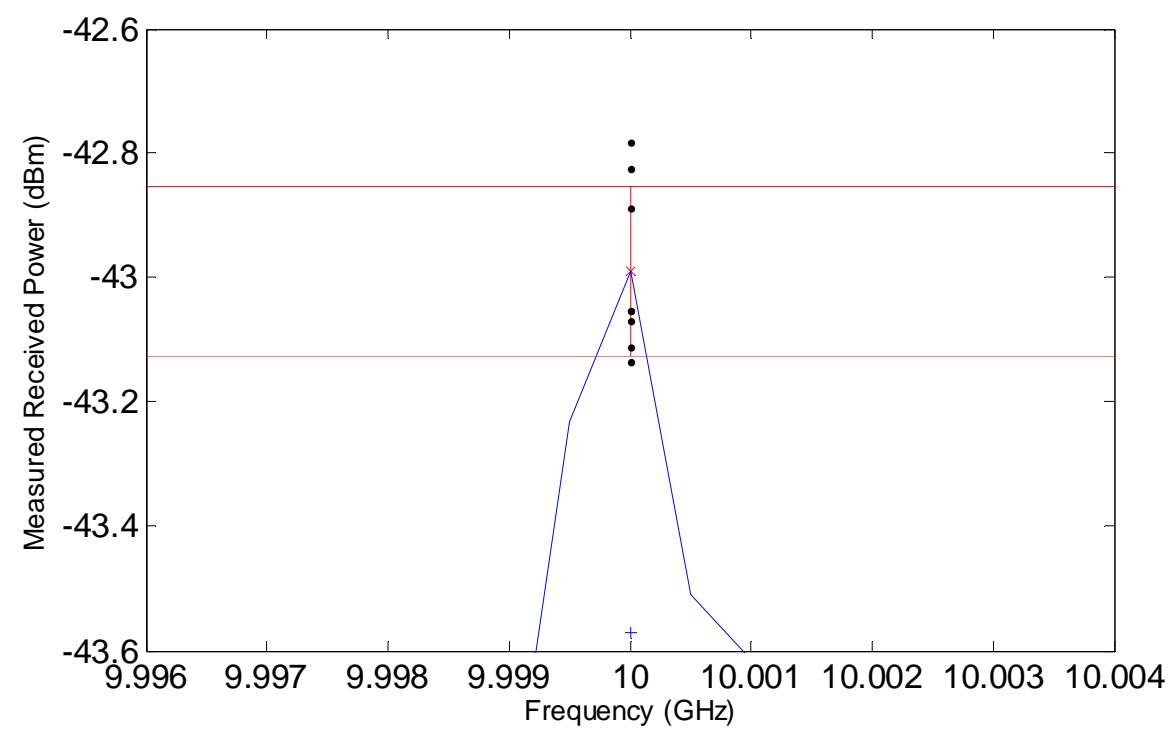

Figure 35. Scaled View of Figure 31 at $10 \mathrm{GHz}$ 


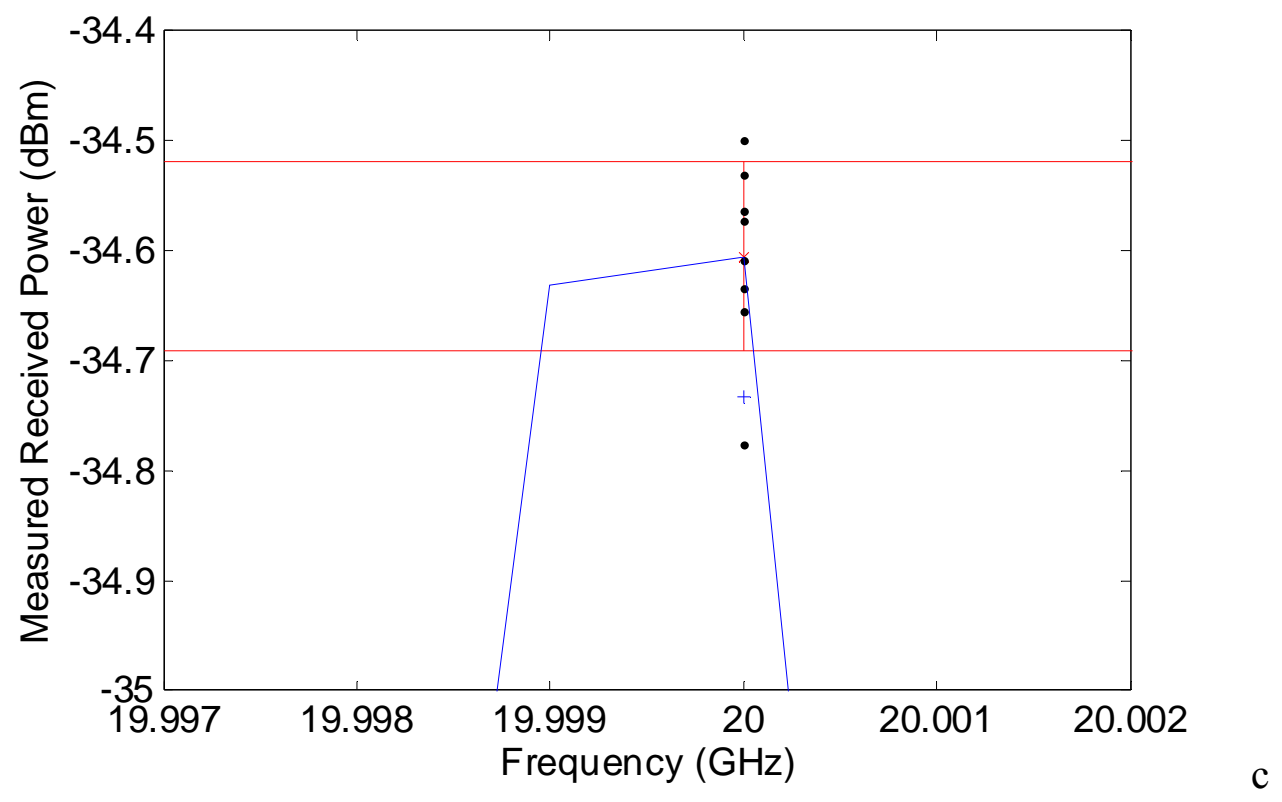

Figure 36. Scaled View of Figure 32 at $20 \mathrm{GHz}$

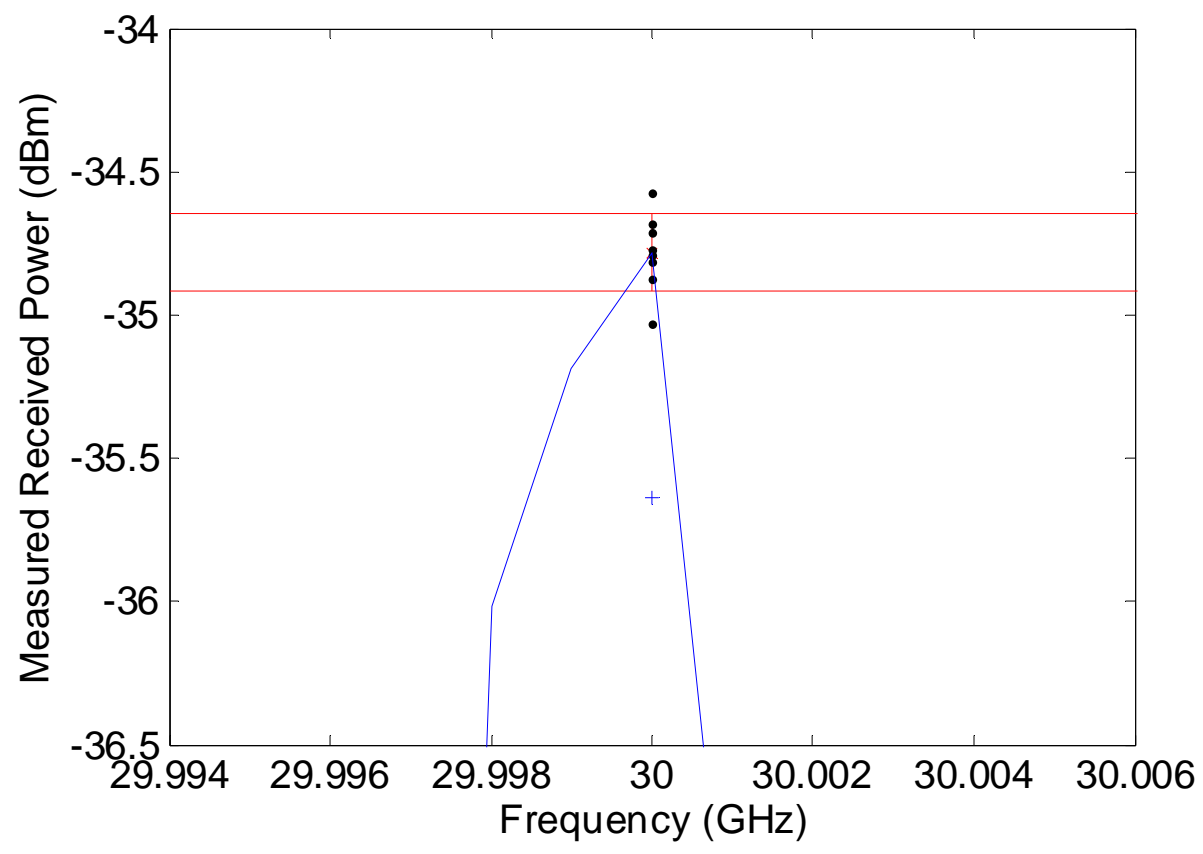

Figure 37. Scaled View of Figure 33 at $30 \mathrm{GHz}$ 


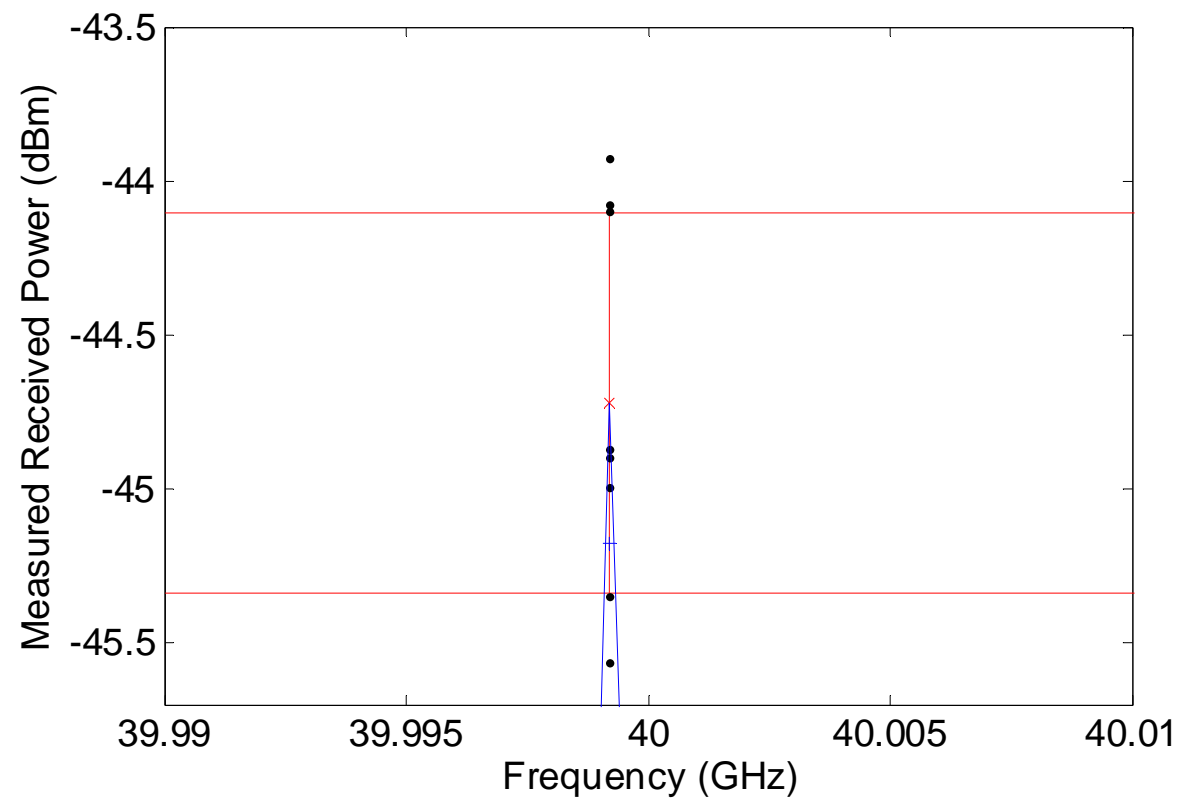

Figure 38. Scaled View of Figure 34 at $40 \mathrm{GHz}$

In Figure 36, the predicted value seems to be estimated as low once again. In this case though, the average measurement value is -34.6 and all of the measurements have standard deviation of 0.081 . The small standard deviation indicates how closely the measured values are packed. Revisiting the figure, the predicted value is only about $0.075 \mathrm{~dB}$ out of the measurement zone and $0.13 \mathrm{~dB}$ away from the average. Although it would be ideal if the predicted value fell in the measurements spread, the raw difference is very small.

In Figure 37, the measurements at $30 \mathrm{GHz}$ are compared to the predicted model. Along with having the largest deviation from the average of $0.85 \mathrm{~dB}$ of all four frequencies, the predicted received power at $30 \mathrm{GHz}$ was also farthest outside the measurement spread. As with the prior two frequencies, the predicted power is lower than the measured values. In this particular case, the predicted power is about $0.75 \mathrm{~dB}$ outside of the measurement spread.

In Figure 38, the model prediction is compared to the measurements at $40 \mathrm{GHz}$. This happened to be the only case in which the prediction method fell within the error limits. Though this is good news, the standard deviation of 0.577 should be evaluated. While it is expected that higher frequencies will have more anomalies associated with them due to their higher susceptibility to losses, a smaller standard deviation should be expected. For now, the data 
suggests that the model is accurate at $40 \mathrm{GHz}$ in the ideal scenario. A test to ensure this would be to take anywhere from 20-50 measurements in the same scenario to achieve a better convergence of data.

In Figure 39, the received powers and the predicted powers were normalized to their respective average powers. In the same manner as before, the error bars were determined by the standard deviation of the normalized powers. The data that is associated with this figure can be seen in Table 22. The point that was made about the spread being too large at $40 \mathrm{GHz}$ can clearly been seen here as it is 2-3 times larger than that of other frequencies.

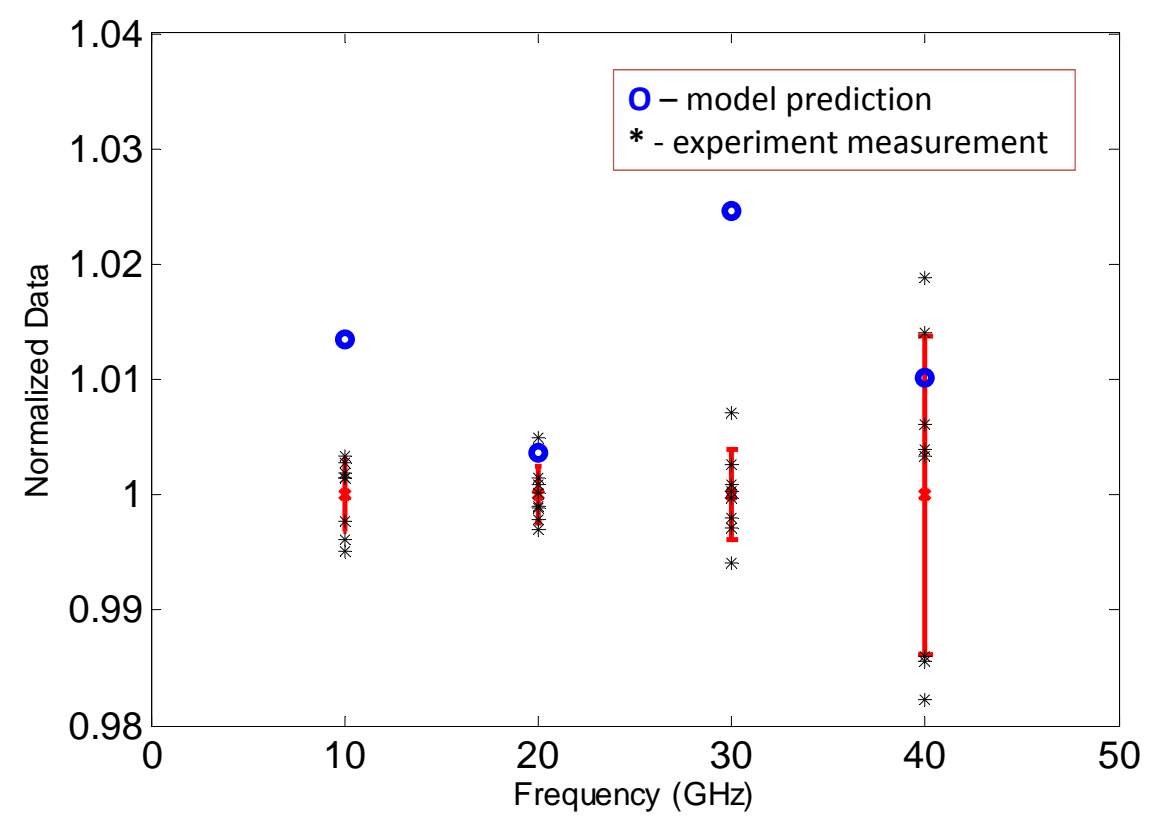

\section{Figure 39. Comparing Measurement Spreads and Model Accuracy at the Four Examined Frequencies}

Though the predicted data is relatively close to the measurements at all frequencies, it still deviates away from the measured value zone. There are several possible explanations for this. The initial though was that the antennas were not properly aligned. Pointing losses were not accounted for, but additional losses would further reduce the power expected rather than bring it closer to the measurements. 
The first possibility for the mismatch is that the antenna gains do not exactly match their specification sheet. In the model, the gain specified is the gain used. Unless the antenna gain is measured exactly, then a possibility of error could always be present.

The second and more probable reason behind the mismatch is that the channel lengths were not measured precisely enough. This could have happened since the measuring tape was not nearly long enough to measure the channel length all at once. So a couple of measurements were taken and added together. The other possibility is that the tape was not pulled tightly enough when measuring, which would allow for a dip in the tape, and ultimately creating a longer distance. Given that the channel length is already small, any minute differences could have an impact on the measurements. For example, the difference of $0.5 \mathrm{~dB}$ could occur if the channel length measured was actually a one foot longer than the actual length.

The third possibility for the inaccuracy is that the cable losses used in the model were not truly representative of the cables used for the experiment. In this particular case, a network analyzer was not available to measure the losses and the cables were not allowed to leave the testing facility. This resulted in having to use a manufacturer specification for the cables used and predict the losses according to their length. This could result in either too much or not enough losses accounted for.

Ultimately, this experiment should have been performed again to either replicate results or achieve more accurate ones. If this experiment were to be attempted again, the designer should take many more measurements at all frequencies to have a solid data set for analysis. More data would negate the possibilities that the measurements did not converge. If high precision is needed, then all antenna gains, feed losses, and cable losses need to be precisely measured and accounted for.

\subsection{Experiment 3 - Quantifying Model Accuracy with Rain Impairment}

Following the anechoic chamber experiment, the results were thought to be decent enough to continue on to the rain modeling. The goal of this experiment was to measure the propagation losses across the plot at $10 \mathrm{GHz}$ prior to, during, and following the rain simulation. Noticeable losses would ideally be measured and the results would match the link-budget model.

Several measurements prior to, during, and following the rain experiment were taken at $10 \mathrm{GHz}$. The idea was that since the ground was in such close proximity to the transmission 
system, water would accumulate and cause signal power to vary throughout as the refractivity of the ground changes. Therefore taking measurements before and after the rainfall should provide a rough approximation of how the index of refraction varied throughout if the measurements varied as well. For reference, the predictive link-budget input parameters and expected link budget calculations can be found in Table 12, Table 13, and Table 14. The measured values, predictive power, and average measured power are plotted in Figure 40, Figure 41, and Figure 42 for the three different scenarios. The data associated with these figures can be seen in Table 23 .

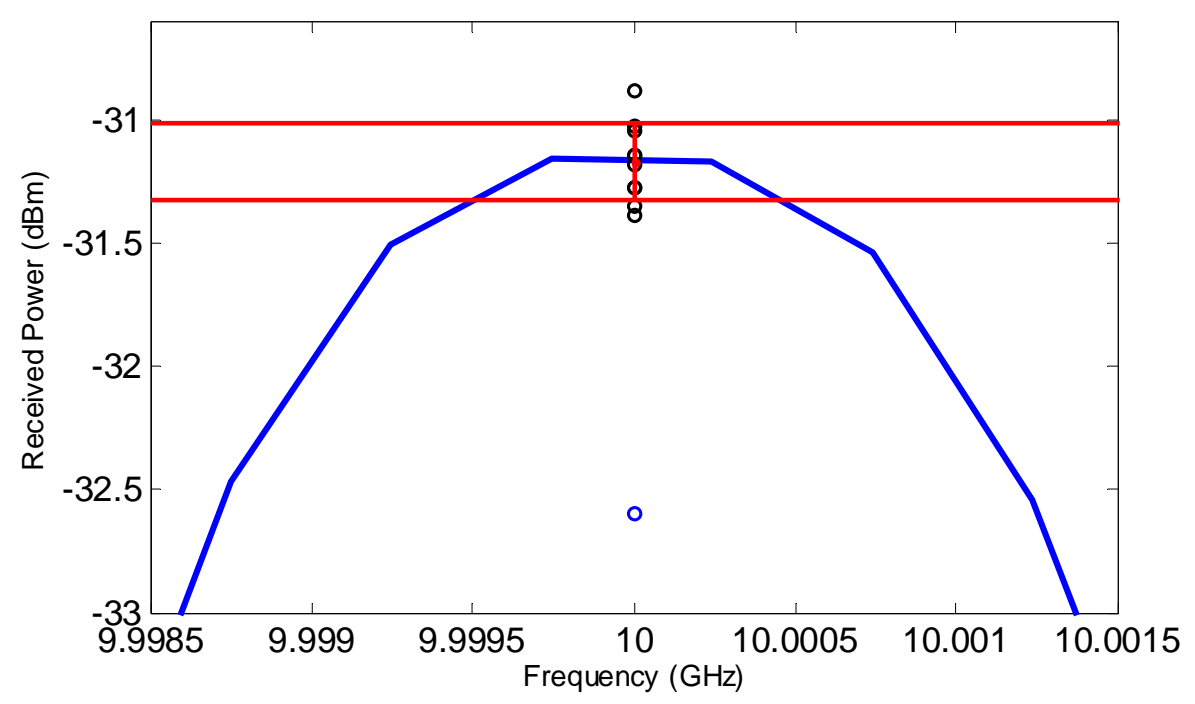

Figure 40. Analyzing the Measurements Prior to Rainfall and Comparing Them to the Predicted Value 


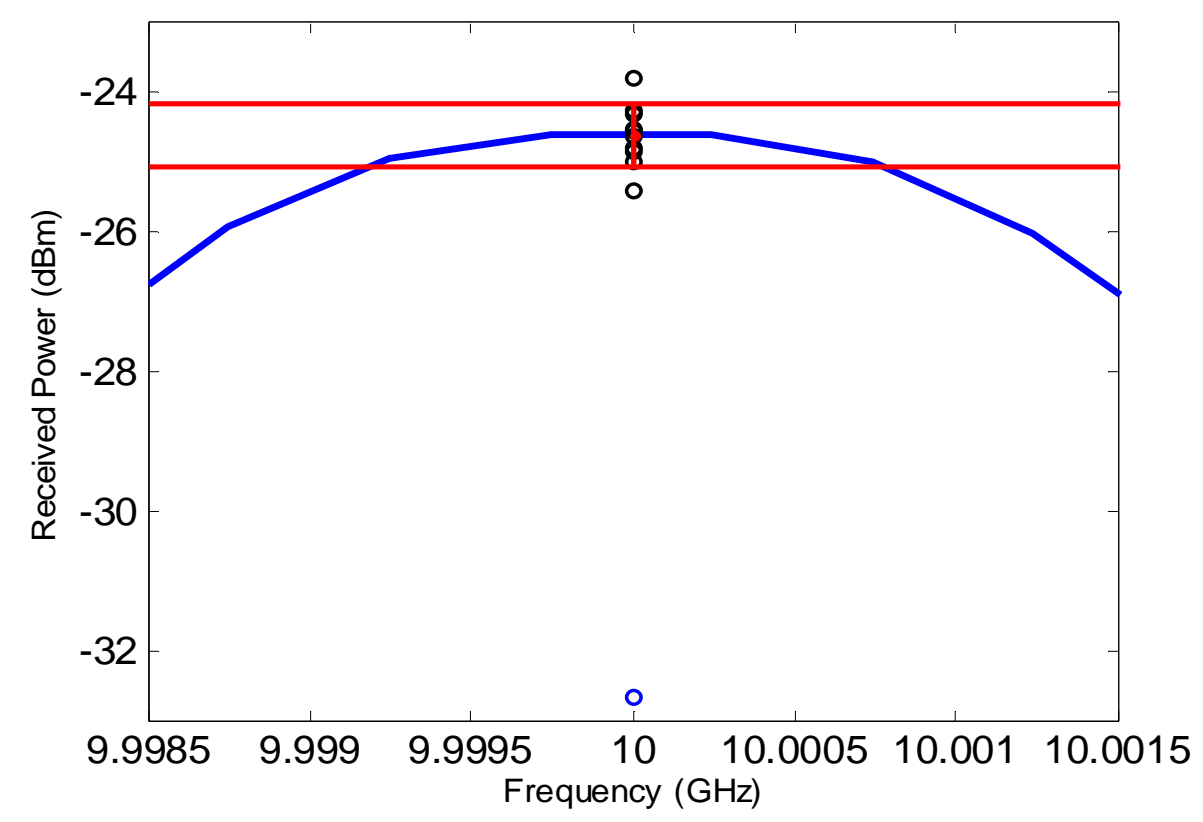

Figure 41. Analyzing the Measurements with Rainfall and Comparing Them to the Predicted Value

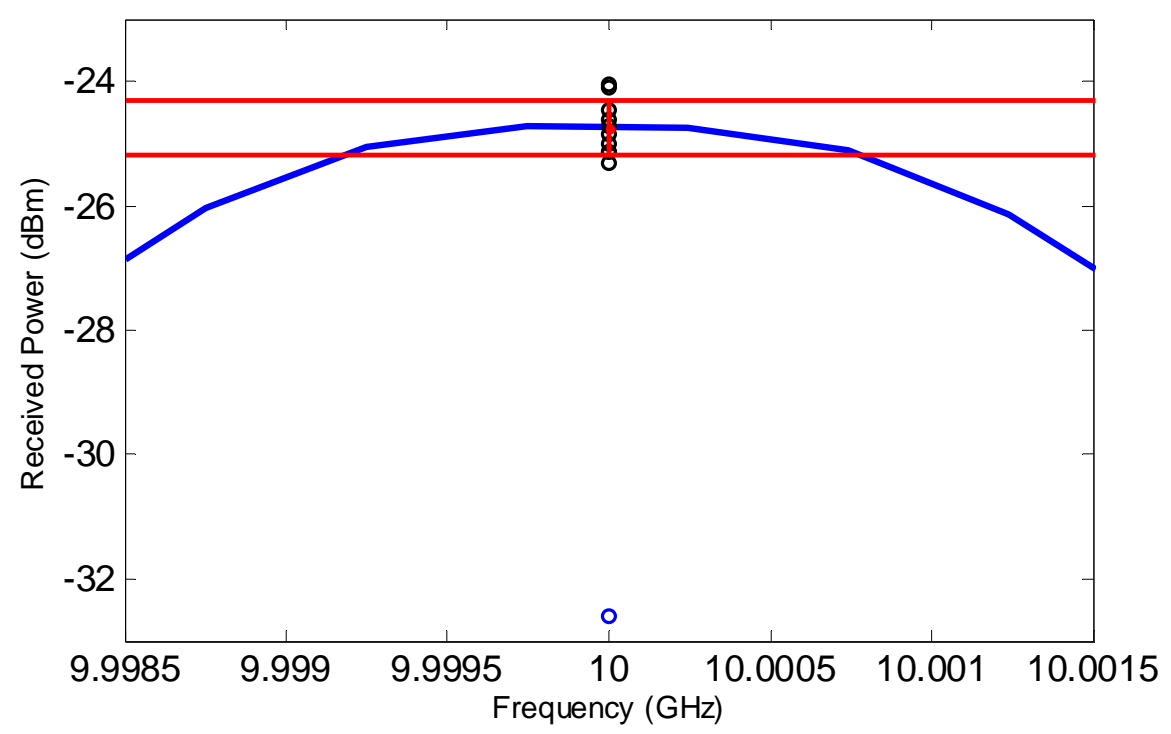

Figure 42. Analyzing the Measurements Post-Rainfall and Comparing Them to the Predicted Value 
Table 23. Measured and Predicted Data for Clear-Sky and Rain Impairments

\begin{tabular}{|c|c|c|c|}
\hline & Before & During & After \\
\hline $\begin{array}{c}\text { Frequency } \\
(\mathbf{H z})\end{array}$ & 10000 & 10000 & 10000 \\
\hline Meas. 1 & -31.18 & -23.80 & -24.47 \\
\hline Meas. 2 & -31.27 & -24.30 & -24.04 \\
\hline Meas. 3 & -30.88 & -24.26 & -24.10 \\
\hline Meas. 4 & -31.14 & -24.56 & -24.61 \\
\hline Meas. 5 & -31.14 & -24.79 & -25.01 \\
\hline Meas. 6 & -31.34 & -24.63 & -24.73 \\
\hline Meas. 7 & -31.27 & -25.42 & -25.14 \\
\hline Meas. 8 & -31.02 & -24.53 & -24.85 \\
\hline Meas. 9 & -31.38 & -24.99 & -25.315 \\
\hline Meas. 10 & -31.04 & -24.85 & -25.12 \\
\hline Model & -32.60 & -32.68 & -32.60 \\
\hline
\end{tabular}

To begin the analysis, a simple difference between the measured averages and the predictive powers is necessary. These can be seen in Table 24. A standard deviation calculation is also present in the same table. From Table 24, there is an immediate concern with the differences between the average data and the predicted data. Specifically, the differences during rainfall and following that are over $7 \mathrm{~dB}$ from the measured average. This was of a major concern considering that the error was present not just during the rainfall. The interesting thing to note is that the power received seemed to have improved $7 \mathrm{~dB}$ during rainfall and after that. At the time of experimentation, this was not noticed. Unfortunately, received power increasing naturally does not happen without cause. It is believed that this fault was due to operator error, though nothing is for certain. That being said, this data set was determined to be invalid, though the possible reasons should be discussed. 
Table 24. Statistical Calculations of the Measured Data

\begin{tabular}{|c|c|c|c|}
\hline & Before & During & After \\
\hline Avg. (dB) & -31.17 & -24.61 & -24.74 \\
\hline Predicted (dB) & -32.60 & -31.68 & -32.60 \\
\hline Diff. (dB) & 1.43196 & 7.0678 & 7.8617 \\
\hline Std. Dev. & 0.14865 & 0.42121 & 0.41201 \\
\hline
\end{tabular}

An in-depth analysis for this experiment was not necessary since there was no guarantee that the data recorded was accurate. Though, the experiment was not a complete loss. Some lessons that were learned were applied to the final experiment. Though the rain rate was at 20 $\mathrm{mm} /$ hour for this experiment, it only operated at that rate for approximately 15 minutes. This made recording measurements extremely difficult without disturbing the biologist's experiments. Had more than one person been there to assist with the setup and data measurements, the faulty data would have probably been discovered prior to recording.

Another unexpected issue that came up was that small breezes created a mist with the rainfall and began to fall on the transmission system. This issue was very quickly resolved. During another occasion, the transmitter was incorrectly setup and had to be corrected quickly in order to continue on with the experimentation. The point of stating all of this is that there was too much to manage for one operator. A possibility for the errors is that during the quick interventions, instruments could have been bumped and accidently altered, which could result in faulty data recorded.

\subsection{Experiment 4 - Quantifying Model Accuracy with Rain Impairment Again}

The goal of this final experiment was to record measureable losses that could be compared against the coupled model. The communication links operating at 10,20,30, and 40 $\mathrm{GHz}$ would show the rain loss relationship as expected if everything went according to plan. Unlike the previous experiment, measurements were required at four different frequencies.

The execution had to be precise since it was the last time that the rain simulator would be operational until the spring time. For the last experiment, two people assisted in the setup and operation of the communication links. For reference, the input parameters and associated 
expected results for these measurements are found in Table 15, Table 16, and Table 17. Figure 43 through Figure 57 are plots of the measured data, and some minor analysis which will be discussed.

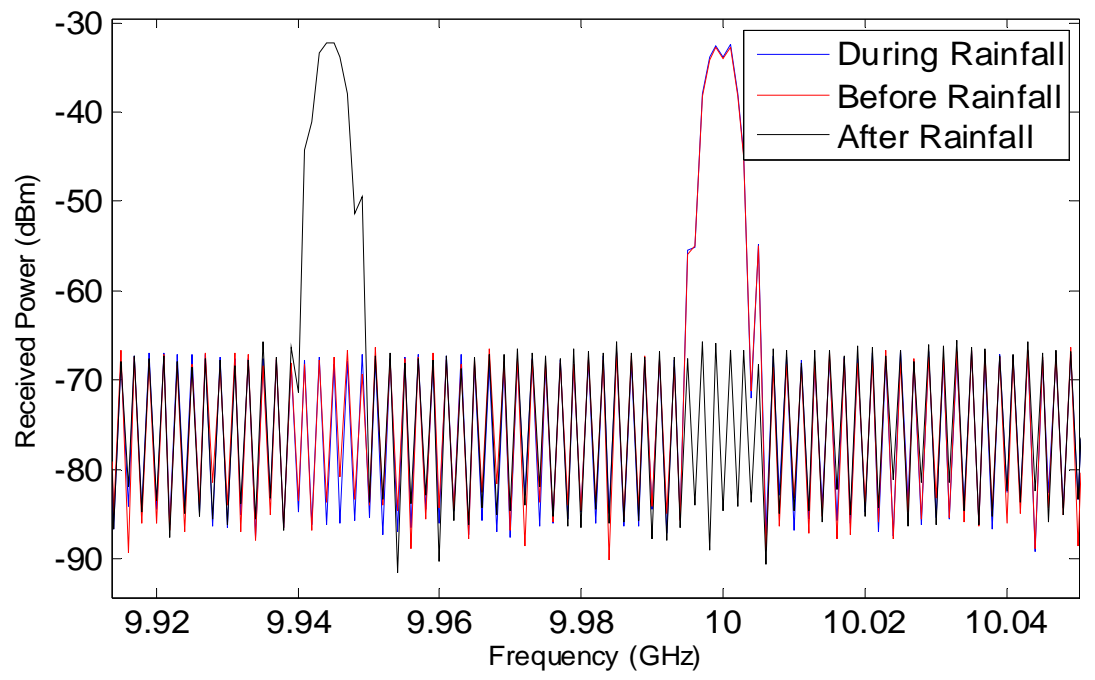

Figure 43. Average Data Sets for Before, During, and After Rainfall for $10 \mathrm{GHz}$

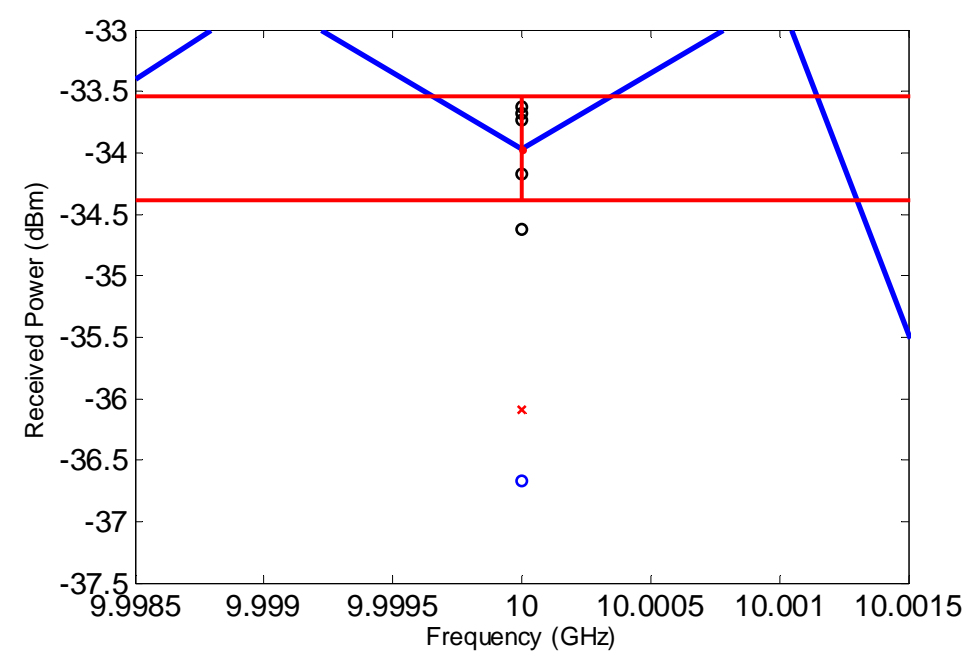

Figure 44. Average Data Set for Before Rainfall for $10 \mathrm{GHz}$ (Showing Measurement Values (Blue 'o') and Predicted Values (Red ' $x$ ')) 


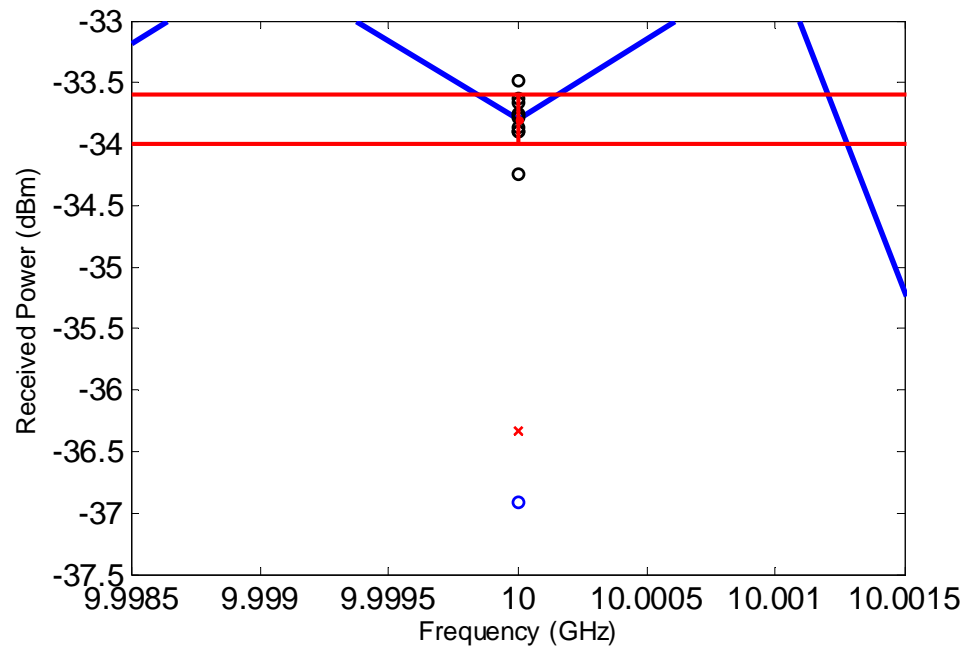

Figure 45. Average Data Set for During Rainfall for $10 \mathrm{GHz}$ (Showing Measurement Values (Blue 'o') and Predicted Values (Red ' $x$ '))

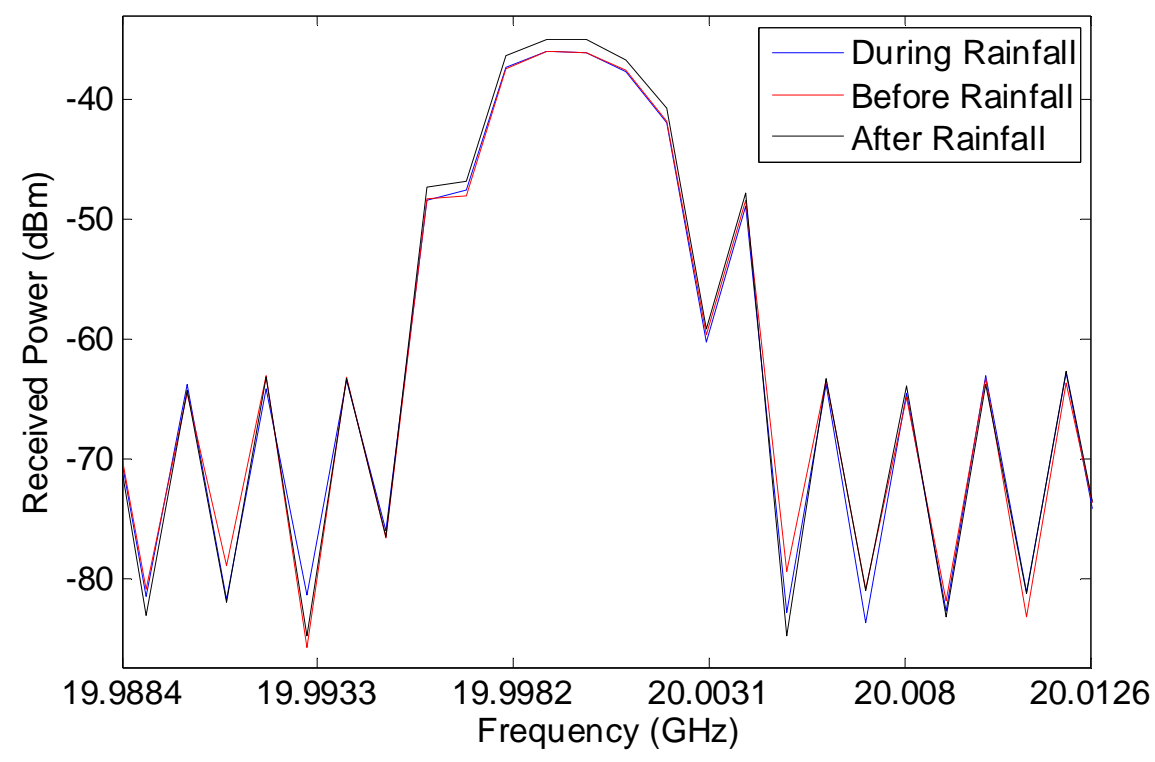

Figure 46. Average Data Sets for Before, During, and After Rainfall for $20 \mathrm{GHz}$ 


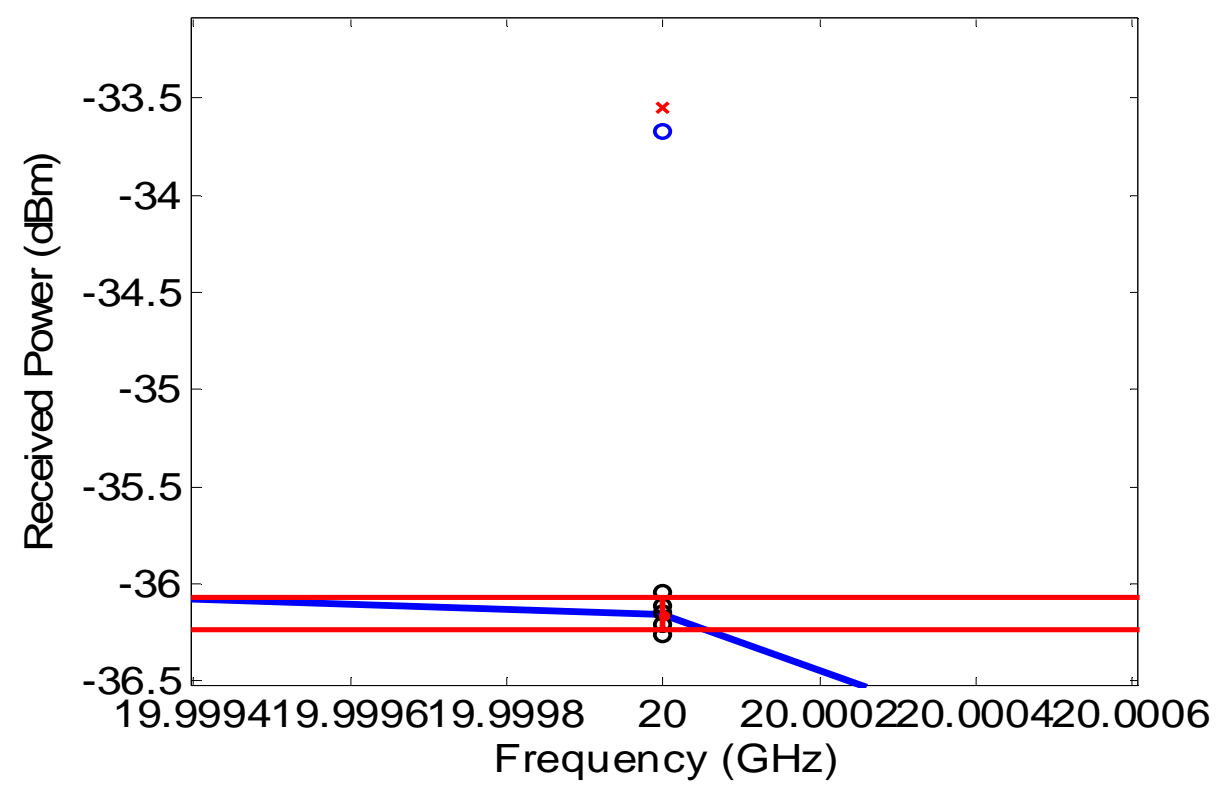

Figure 47. Average Data Set Prior to Rainfall at 20 GHz (Showing Measurement Values (Blue 'o') and Predicted Values (Red ' $x$ '))

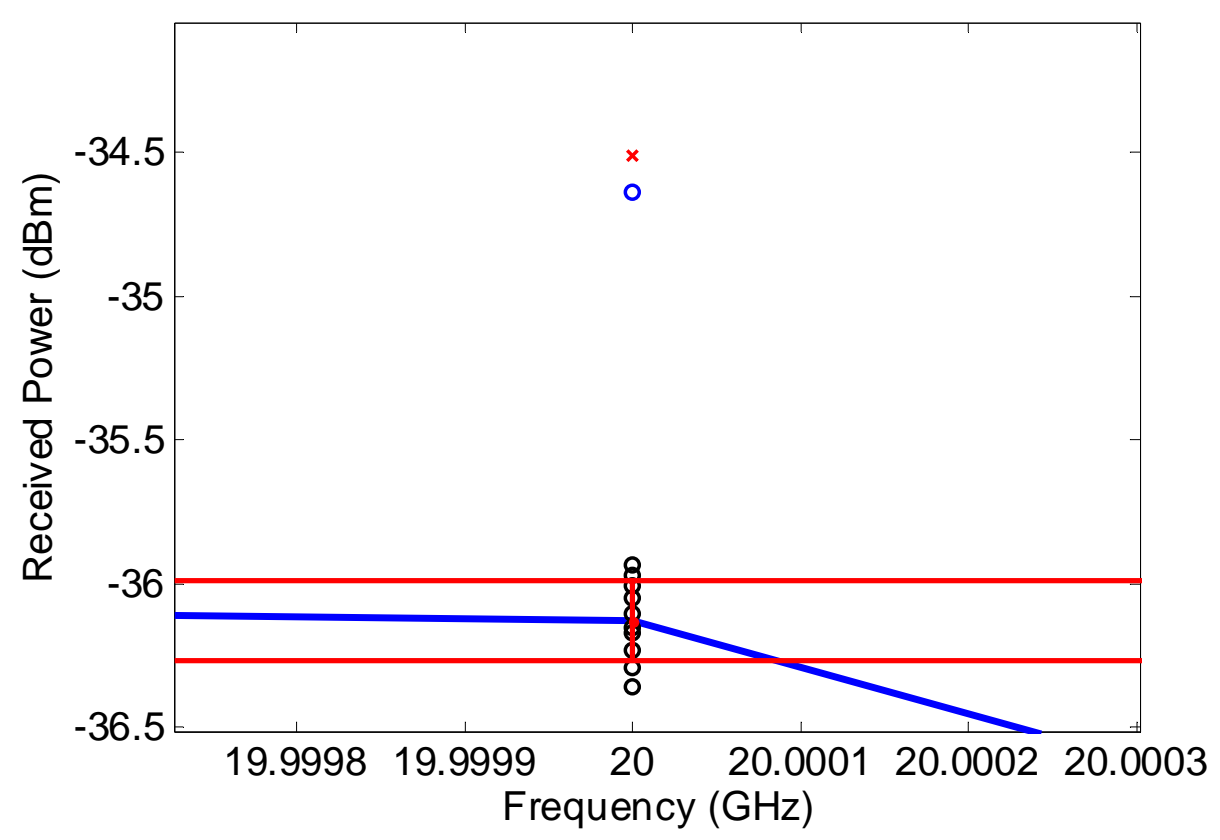

Figure 48. Average Data Set for During Rainfall for $20 \mathrm{GHz}$ (Showing Measurement Values (Blue 'o') and Predicted Values (Red ' $x$ ')) 


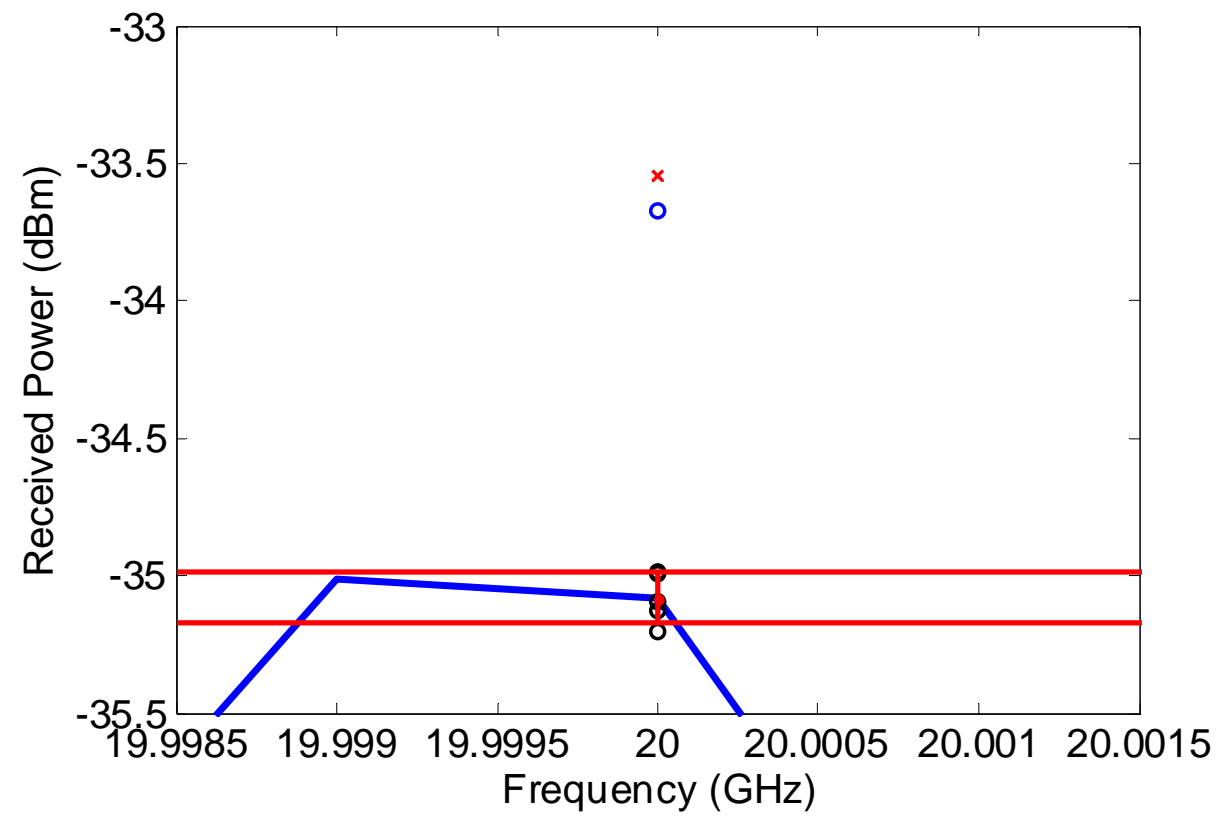

Figure 49. Average Data Set for Post-Rainfall for $20 \mathrm{GHz}$ (Showing Measurement Values (Blue ' 0 ') and Predicted Values (Red ' $x$ '))

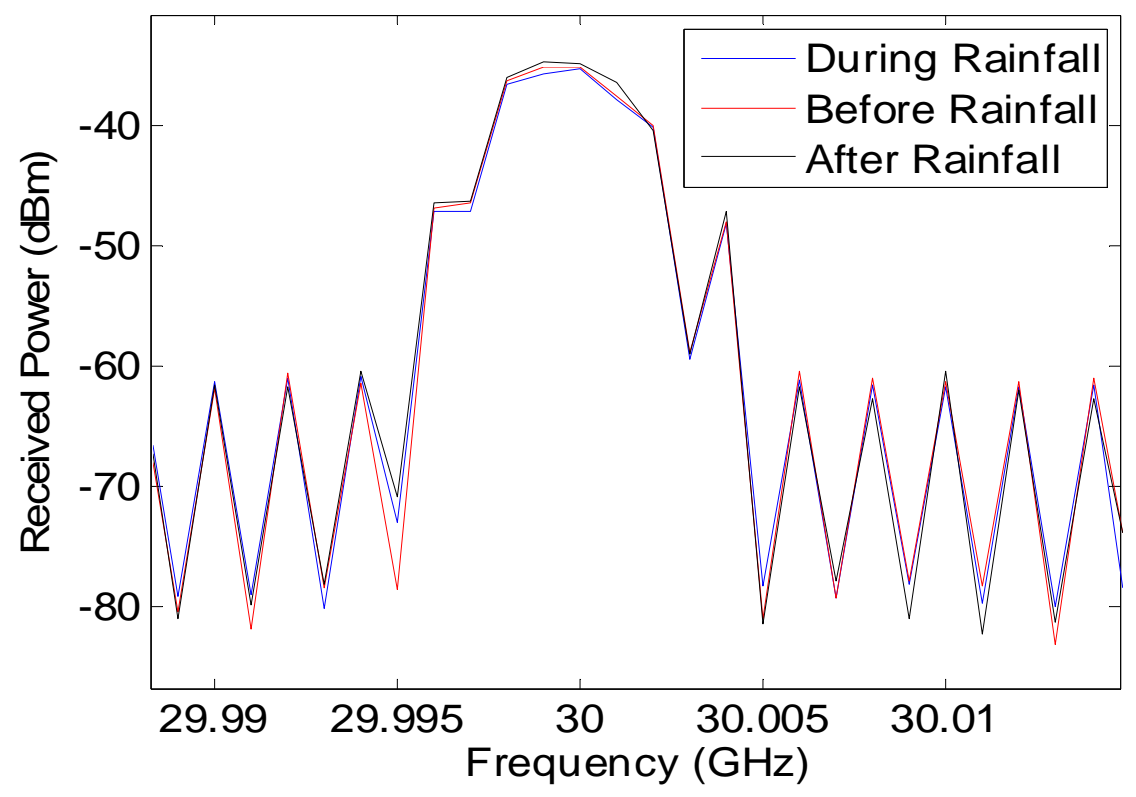

Figure 50. Average Data Sets for Before, During, and After Rainfall for $30 \mathrm{GHz}$ 


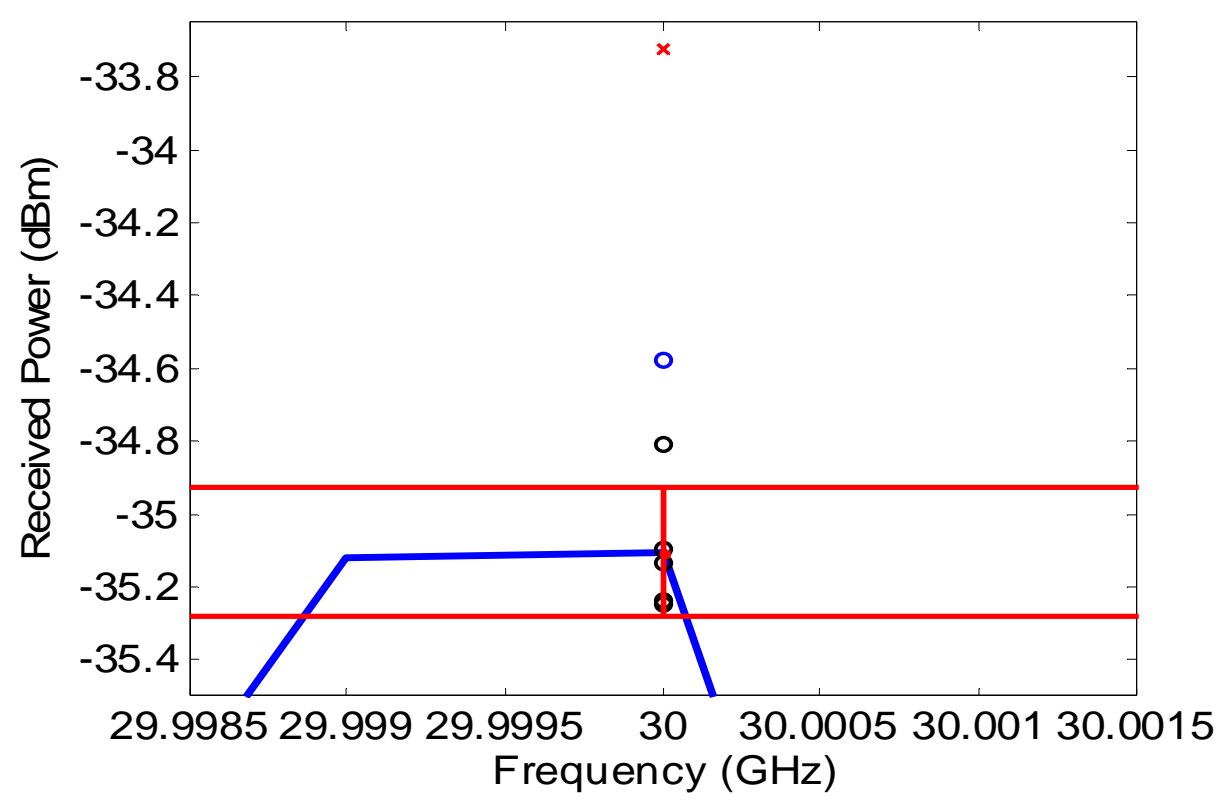

Figure 51. Average Data Set For Before Rainfall for $30 \mathrm{GHz}$ (Showing Measurement Values (Blue 'o') and Predicted Values (Red ' $x$ '))

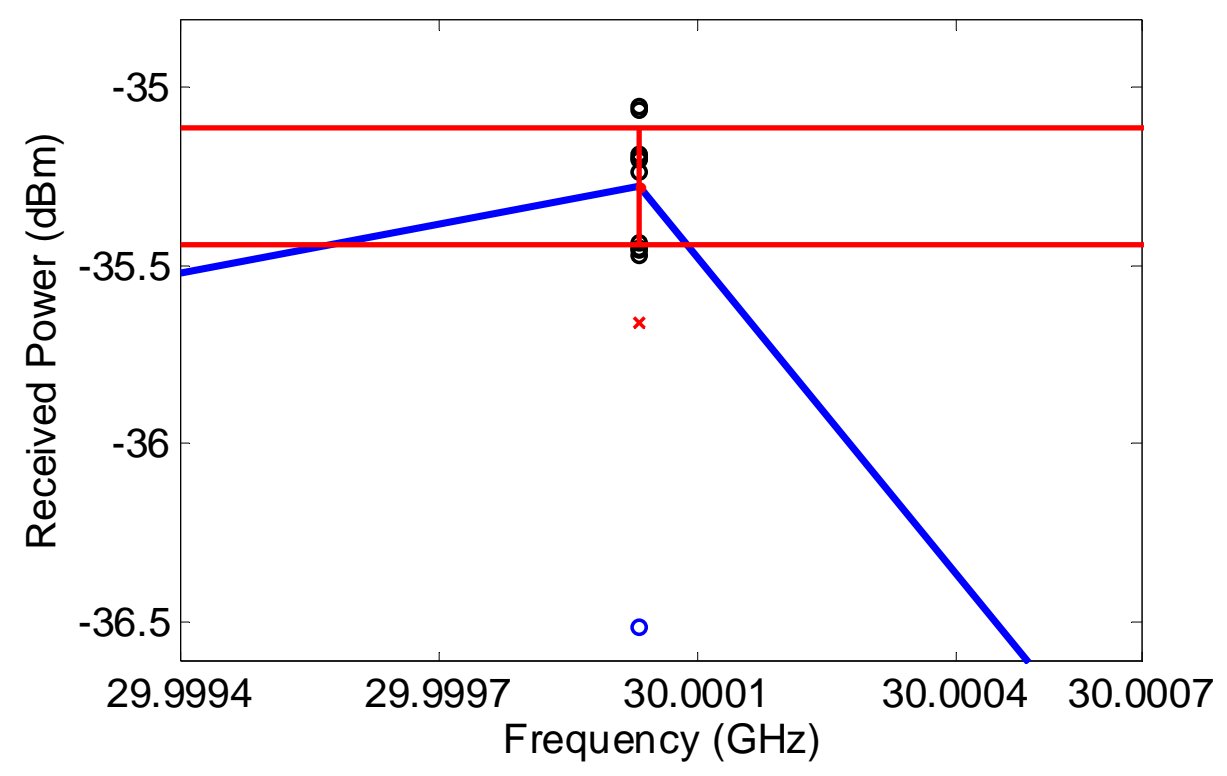

Figure 52. Average Data Set For During Rainfall for $30 \mathrm{GHz}$ (Showing Measurement Values (Blue 'o') and Predicted Values (Red ' $x$ ')) 


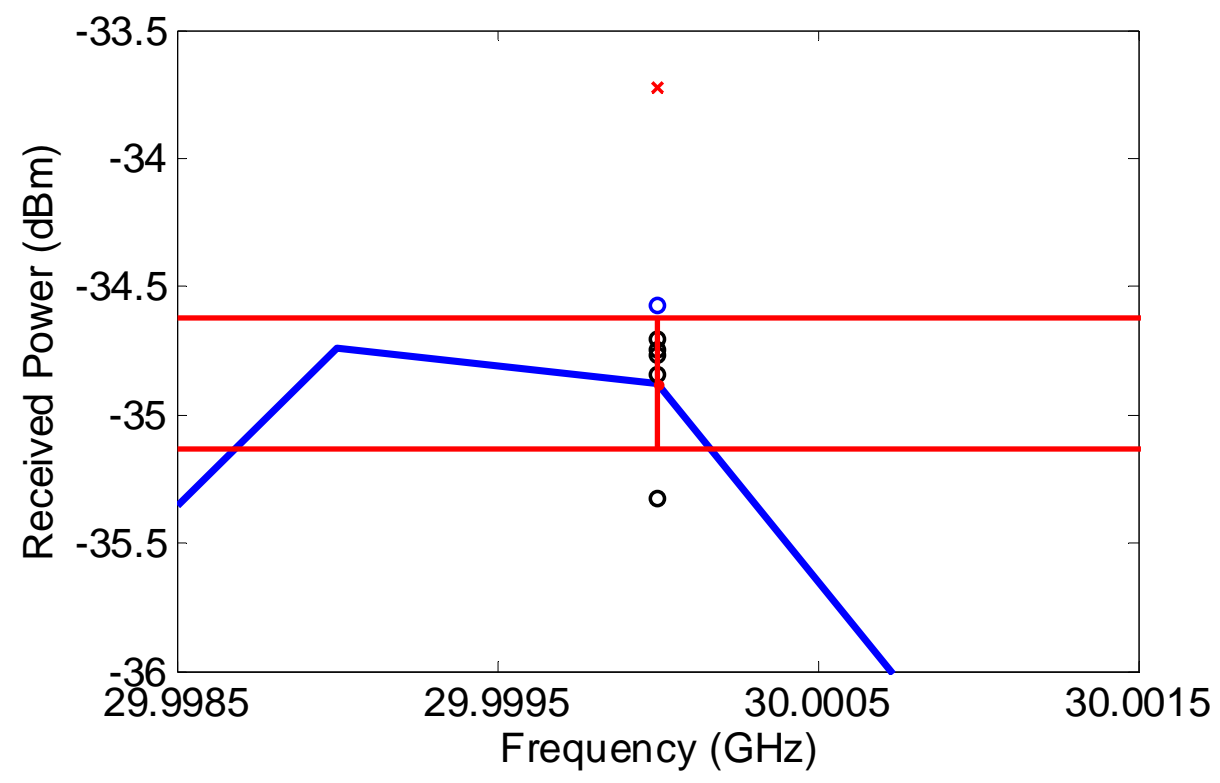

Figure 53. Average Data Set For Post-Rainfall for $30 \mathrm{GHz}$ (Showing Measurement Values (Blue 'o') and Predicted Values (Red ' $x$ '))

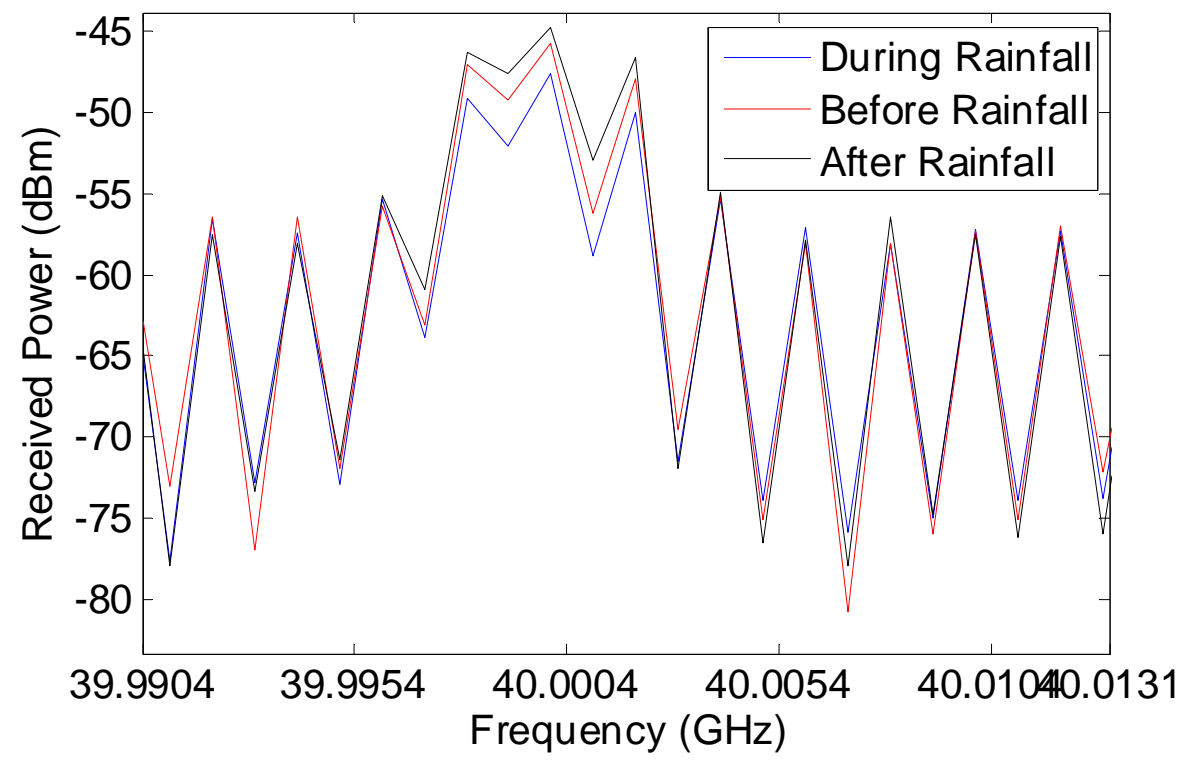

Figure 54. Average Data Sets For Before, During, and After Rainfall for $40 \mathrm{GHz}$ 


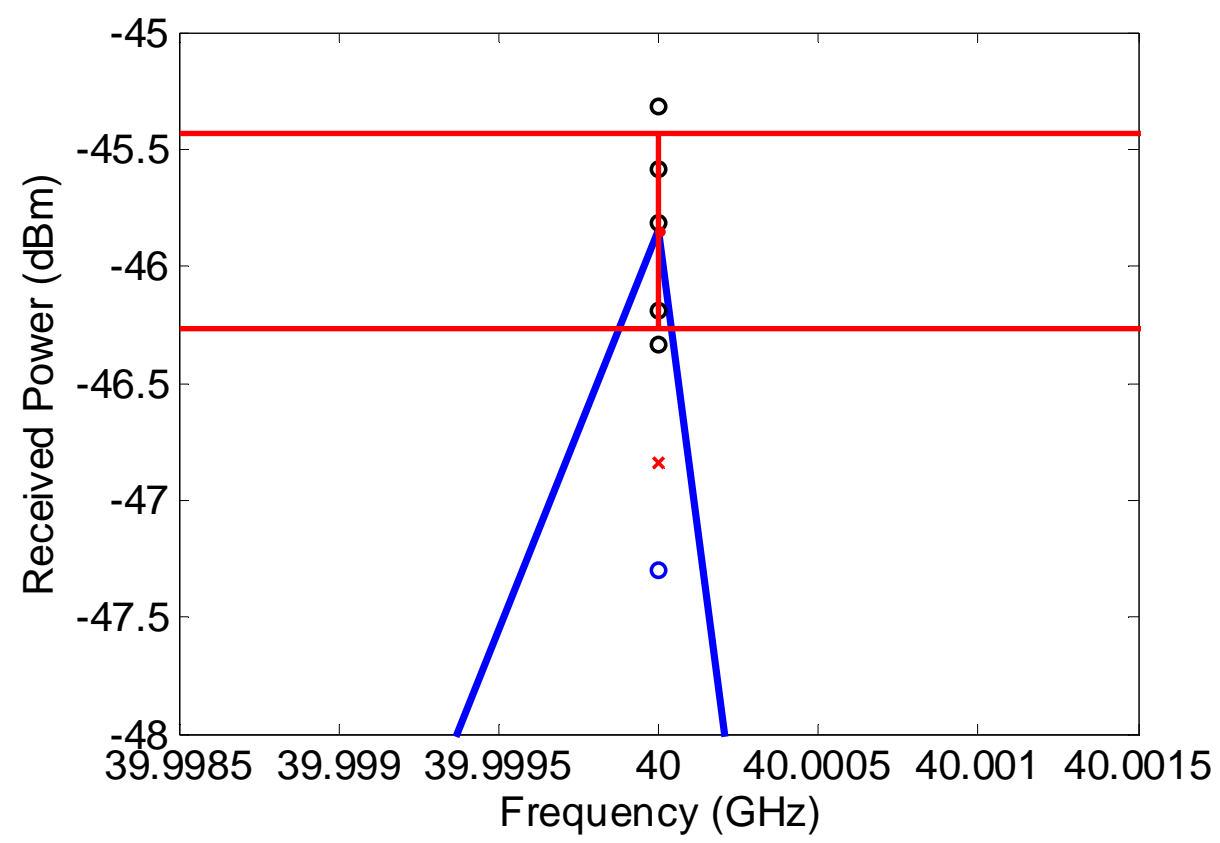

Figure 55. Average Data Set for Before Rainfall for $40 \mathrm{GHz}$ (Showing Measurement Values (Blue 'o') and Predicted Values (Red ' $x$ '))

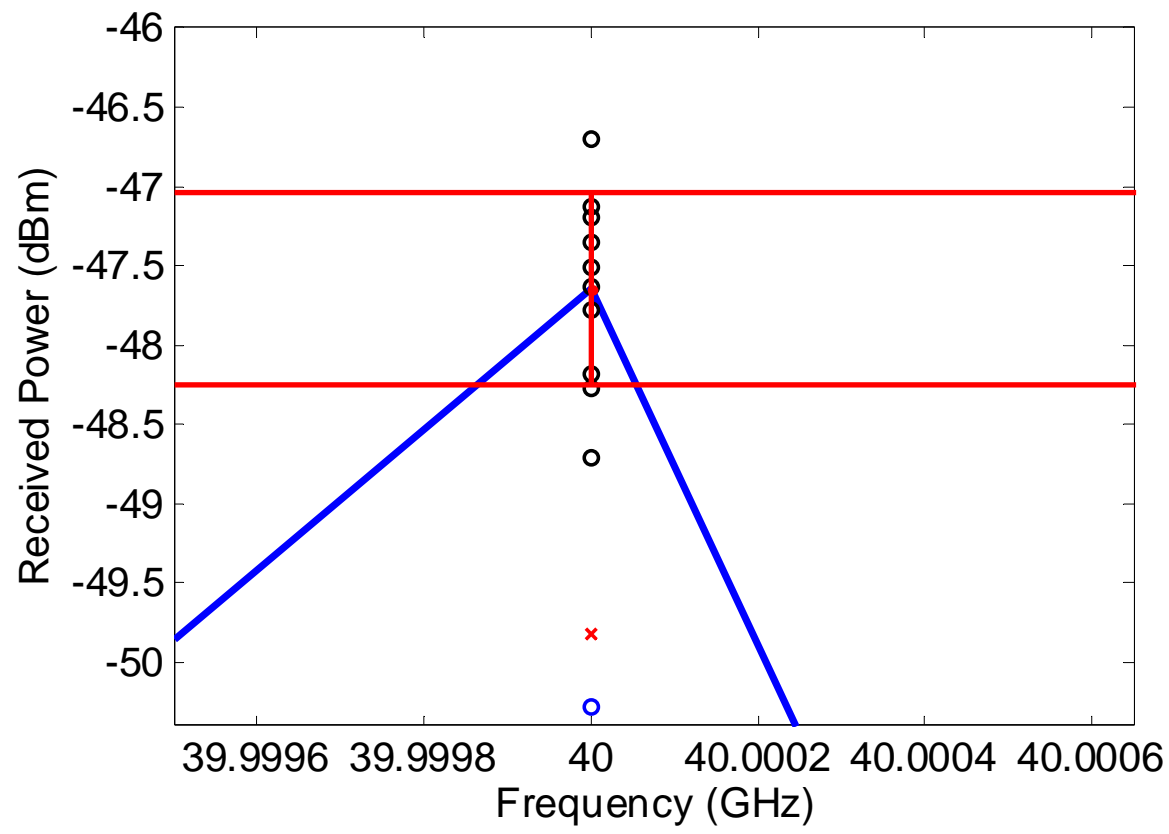

Figure 56. Average Data Set for During Rainfall for $40 \mathrm{GHz}$ (Showing Measurement Values (Blue 'o') and Predicted Values (Red ' $x$ '))

Approved for Public Release; Distribution is Unlimited. 


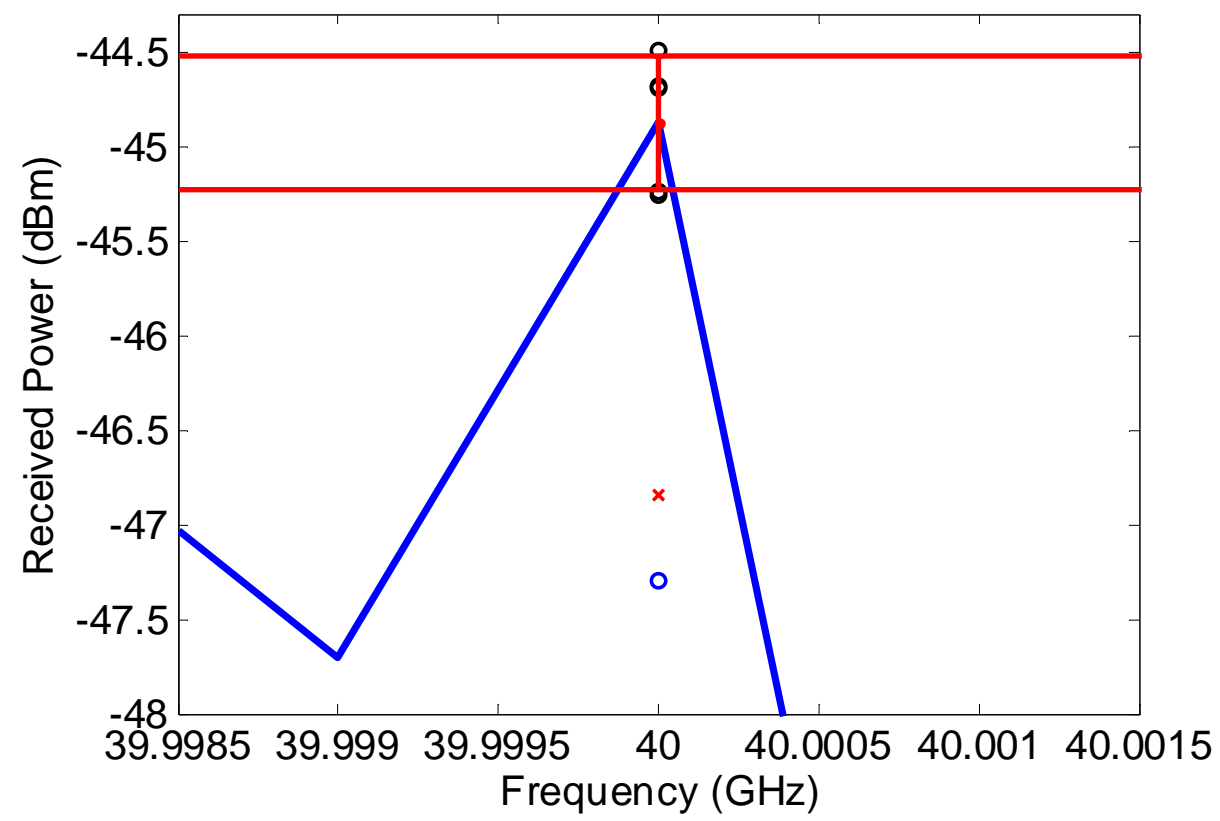

Figure 57. Average Data Set For Post-Rainfall for 40 GHz (Showing Measurement Values (Blue 'o') and Predicted Values (Red ' $x$ '))

In this final experiment, a lot of data was taken as can be seen by the many figures. As mentioned previously, the idea behind taking three different data sets at each frequency (before, during, and after rainfall) was that a variance in the data before and after would occur. If noticeable differences were detected, then a correction factor could be determine and utilized to account for this issue.

The real idea was that the accumulated water on the ground would result in the refractive index of the ground changing, thus resulting in signal variance throughout. Though this is only a hypothesis, it was desired that data would still be taken in this manner for later analysis. Normally a data set like this would not require any modifications. Since the propagation link is close to the ground, it begins to stray farther away from a free-space scenario. Once it does this, then losses endured by the signal cannot be associated with specific attenuators since the environment presents more variables.

Table 25 through Table 29 show not all of the data is present. This is the case due to the fact that the transmitter was accidently adjusted following the transmission through the rainfall. 
In Figure 43, this is clear since a different spike is present to the left of the other data sets. Though data was recorded, it was decided that the data was not truly representative of a $10 \mathrm{GHz}$ signal since data accuracy is of high importance. One could argue that the losses expected would be very similar to the $10 \mathrm{GHz}$ signal. It is true that the losses would be similar, but they would not be the same. When characterizing a model, it is of the upmost importance that the data being used is as accurate as possible.

Table 25. 10 GHz Data Sets Used for Statistical Analysis

\begin{tabular}{|c|c|}
\hline Before (dB) & During (dB) \\
\hline-34.62 & -33.49 \\
\hline-34.17 & -33.67 \\
\hline-33.74 & -33.90 \\
\hline-33.62 & -34.24 \\
\hline-33.68 & -33.63 \\
\hline-33.97 & -33.76 \\
\hline & -33.76 \\
\hline & -33.79 \\
\hline & -33.86 \\
\hline & -33.90 \\
\hline
\end{tabular}


Table 26. 20 GHz Data Sets Later Used for Statistical Analysis

\begin{tabular}{|c|c|c|}
\hline Before (dB) & $\begin{array}{c}\text { During } \\
(\mathbf{d B})\end{array}$ & After (dB) \\
\hline-36.11 & -36.12 & -35.13 \\
\hline-36.15 & -36.05 & -35.10 \\
\hline-36.27 & -36.36 & -35.20 \\
\hline-36.21 & -36.01 & -34.99 \\
\hline-36.04 & -36.15 & -34.99 \\
\hline & -36.29 & \\
\hline & -36.17 & \\
\hline & -36.23 & \\
\hline & -35.98 & \\
\hline & -35.94 & \\
\hline
\end{tabular}

Table 27. Simple Table Conveying the Average Measured Power and the Predicted Measured Power

\begin{tabular}{|c|c|c|c|c|}
\hline Frequency (GHz) & $\mathbf{1 0}$ & $\mathbf{2 0}$ & $\mathbf{3 0}$ & $\mathbf{4 0}$ \\
\hline Rain Predicted (dB) & -36.92 & -34.64 & -36.51 & -50.28 \\
\hline Rain Meas. (dB) & -33.97 & -36.13 & -35.28 & -47.65 \\
\hline
\end{tabular}


Table 28. 30 GHz Data Sets Later Used for Statistical Analysis

\begin{tabular}{|c|c|c|}
\hline Before (dB) & $\begin{array}{c}\text { During } \\
(\mathbf{d B})\end{array}$ & After (dB) \\
\hline-35.14 & -35.06 & -34.71 \\
\hline-35.25 & -35.24 & -34.84 \\
\hline-35.10 & -35.47 & -34.77 \\
\hline-35.24 & -35.45 & -34.75 \\
\hline-34.81 & -35.20 & -35.33 \\
\hline & -35.06 & \\
\hline & -35.46 & \\
\hline & -35.20 & \\
\hline & -35.19 & \\
\hline & -35.44 & \\
\hline
\end{tabular}

Table 29. 40 GHz Data Sets Later Used for Statistical Analysis

\begin{tabular}{|c|c|c|}
\hline Before (dB) & $\begin{array}{c}\text { During } \\
(\mathbf{d B})\end{array}$ & After (dB) \\
\hline-45.81 & -48.71 & -45.24 \\
\hline-45.32 & -46.70 & -44.68 \\
\hline-45.59 & -47.35 & -44.69 \\
\hline-46.33 & -47.63 & -45.26 \\
\hline-46.20 & -47.52 & -44.49 \\
\hline & -48.28 & \\
\hline & -48.18 & \\
\hline & -47.20 & \\
\hline & -47.13 & \\
\hline & -47.78 & \\
\hline
\end{tabular}


As mentioned above, one of the main goals of this experiment was to record measurable differences between a clear-sky environment, and an environment with rain present. This can be seen in Table 30. As in the previous experiments, statistical data will assist in the evaluation of the model and the measured data. This can be seen in Table 31 and Table 32 for rain and clearsky measurements respectively.

Table 30. Measured Data Comparison to Ensure That Losses Were Indeed Present During Rainfall

\begin{tabular}{|c|c|c|c|c|}
\hline Frequency (GHz) & $\mathbf{1 0}$ & $\mathbf{2 0}$ & $\mathbf{3 0}$ & $\mathbf{4 0}$ \\
\hline Clear Sky Meas. Pre Rain (dB) & -33.80 & -36.16 & -35.11 & -45.85 \\
\hline Rain Meas. (dB) & -33.97 & -36.13 & -35.28 & -47.65 \\
\hline Clear Sky Meas. Post Rain (dB) & - & -35.08 & -34.88 & -44.87 \\
\hline Diff. Pre-Rain (dB) & 0.167 & -0.027 & 0.171 & 1.798 \\
\hline Diff. Post-Rain (dB) & - & 1.045 & 0.399 & 2.773 \\
\hline
\end{tabular}

Table 31. Statistical Data For All Frequencies During Rainfall

\begin{tabular}{|c|c|c|c|c|}
\hline Frequency (GHz) & $\mathbf{1 0}$ & $\mathbf{2 0}$ & $\mathbf{3 0}$ & $\mathbf{4 0}$ \\
\hline Stan. Dev. & 0.19 & 0.13 & 0.16 & 0.57 \\
\hline Rain Meas. (dB) & -33.97 & -36.13 & -35.28 & -47.65 \\
\hline Rain Predicted (dB) & -36.92 & -34.64 & -36.51 & -50.28 \\
\hline Raw Diff (dB) & -2.95 & 1.49 & -1.24 & -2.63 \\
\hline
\end{tabular}


Table 32. Statistical Data For All Frequencies During The Clear-Sky Scenario

\begin{tabular}{|c|c|c|c|c|c|}
\hline Frequency (GHz) & $\mathbf{1 0}$ & $\mathbf{2 0}$ & $\mathbf{3 0}$ & $\mathbf{4 0}$ & Average \\
\hline Clear Sky Pred. (dB) & -36.67 & -33.68 & -34.58 & -47.30 & - \\
\hline Clear Sky Meas. Pre Rain (dB) & -33.97 & -36.16 & -35.11 & -45.85 & - \\
\hline Clear Sky Meas. Post Rain (dB) & - & -35.08 & -34.88 & -44.87 & - \\
\hline Diff. Pre-Rain (dB) & -2.70 & 2.48 & 0.53 & -1.45 & -0.29 \\
\hline Diff. Post Rain (dB) & - & 1.41 & 0.30 & -2.42 & -0.24 \\
\hline (Pre-Rain)- (Post Rain) (dB) & - & 1.07 & 0.23 & 0.98 & - \\
\hline
\end{tabular}

In Table 30, it is clear that the presence of rain had a detectable impact on the radiowave. Note that all of the measured powers (except $10 \mathrm{GHz}$ due to the lack of data) increased following the rainfall simulation. As mentioned above, the thought was that the addition of "standing water" to the ground resulted in an altered refractive index, and ultimately a more reflective surface. Once again this is only a hypothesis due to the time constraint. The second set of differences is relatively close to the differences expected by the model. The differences expected from the model were $0.247,0.963,1.94$, and $2.98 \mathrm{~dB}$ for $10,20,30$, and $40 \mathrm{GHz}$ respectively. Whereas the measured differences following the rainfall simulation were 1.04, 0.3985, and 2.63 $\mathrm{dB}$ for 20,30 , and $40 \mathrm{GHz}$. With the exception of the difference at $30 \mathrm{GHz}$, the variance between the two is very little.

In Table 32, the data for all frequencies during rainfall is presented. The difference between the average measured value and the predicted value is a little off for all frequencies. It is also unfortunate that the predicted values do not fall within a standard deviation of the average data. Therefore, these require some explanation for being far-off.

Revisiting the anechoic chamber experiment; measureable differences were recorded at each frequency to indicate how far the model is away from ideal. Though no experiments have been performed to see if these differences are scalable to different channel lengths, the difference could possibly be assumed in this case. This might be the case since the channel lengths of both 
experiments are comparable in length, with experiment 2 having a 19-m channel length and experiment 4 having a 17-m channel length.

If in fact these differences are scalable and even somewhat accurate for this channel length, then the errors must be derivative of measurement inaccuracies and other unaccounted losses such as pointing losses and multipath fading. A possible correction factor for this channel length is applied in Table 33. Notice that the majority of the differences slightly improve with the addition of the correction factor. These values can be compared in the figures above as it is indicated by a red " $x$ " whereas the predicted data is labeled as a blue "o". Though this factor has not been fully tested, it could definitely serve as a "work-in-progress" as this project continues on.

Table 33. Statistical Data with Correction Factor

\begin{tabular}{|c|c|c|c|c|}
\hline Frequency (GHz) & $\mathbf{1 0}$ & $\mathbf{2 0}$ & $\mathbf{3 0}$ & $\mathbf{4 0}$ \\
\hline Rain Predicted (dB) & -36.92 & -34.64 & -36.51 & -50.28 \\
\hline Rain Meas. (dB) & -33.97 & -36.13 & -35.28 & -47.65 \\
\hline Raw Diff (dB) & -2.95 & 1.49 & -1.24 & -2.63 \\
\hline Model Corr. (dB) & 0.58 & 0.13 & 0.85 & 0.45 \\
\hline Rain Pred. w/ Corr. (dB) & -36.34 & -34.51 & -35.66 & -49.82 \\
\hline Corr Diff. (dB) & -2.37 & 1.62 & -0.38 & -2.18 \\
\hline
\end{tabular}

In Table 32, the clear-sky data is analyzed. Again the differences present are outside the standard deviation of each measured data set as can be seen in Figure 44, Figure 46, Figure 47, Figure 49, Figure 51, Figure 52, Figure 54, and Figure 56. The most interesting thing about Table 32 is the differences between the data measured before and after the rainfall. In every case, the received power increases following the rain simulation. Ideas of constructive multipath were considered, but not investigated in this thesis.

This rain experiment yielded interesting data across the board. The measurement and model differences were definitely present at all frequencies. Unfortunately, there is not a clear 
understanding for the differences since some data sets are higher than the predicted while others are lower.

Though there is no definite answer, several possibilities for inaccuracies in the measurements and the model should be discussed. The possible error that stands out first is that the hosting experimental team was not positive of the rain rate. Originally, it was stated that the rain fell at $60 \mathrm{~mm} /$ hour accurately. The last time around, the researchers pointed out that their tank was lower than usual and that could alter the rain rates. The key note for future testing is to examine the water tank prior to testing to ensure that the proper amount is present.

As in the first experiment, a possibility of ground reflections was present in this case. This could result in multipath and additional fading or possibly constructive signals. Contributions due to ground reflections, especially near $40 \mathrm{GHz}$ are not really expected since the higher frequencies attenuate easily with interferers. Though generally true, the measured data conveyed that the signal power increased following rainfall. This will have to be further investigated to really prove anything.

As in the other experiments, inaccuracies could be resultant from poor channel measurements, pointing losses, and individual parts that deviate away from the specifications as provided by the manufacturer.

\section{CONCLUSIONS}

In conclusion, the work presented ultimately conveyed an alternative method to measuring propagation losses. From the results obtained, the coupled model proves to be a fair representation of what to expect during free-space and rain impairment situations. Though analysis focused heavily on the difference between the model predictions and the actual data, it is important to logically understand that a $2-3 \mathrm{~dB}$ difference at $40 \mathrm{GHz}$ is not that terrible when planning a link. Typically a designer will plan for a link with enough margin to handle unexpected losses.

The model achieved accuracies of $8.69 \%,-4.13 \%, 3.51 \%$, and $5.52 \%$ for $10,20,30$, and $40 \mathrm{GHz}$ respectively in comparison to the rain models. The model achieved clear-sky accuracies of $7.96 \%,-4.02 \%,-0.863 \%$, and $5.4 \%$ for $10,20,30$, and $40 \mathrm{GHz}$ respectively. After the correction factor was included, the rain model achieved accuracies of $6.98 \%,-4.48 \%, 1.09 \%$, and $4.57 \%$ for $10,20,30$, and $40 \mathrm{GHz}$, respectively. The model then achieved clear-sky accuracies of 
$6.25 \%,-4.38 \%,-3.31 \%$, and $3.3 \%$ for $10,20,30$, and $40 \mathrm{GHz}$ respectively. However, the correction factor's validity has not been decided upon, and therefore it is not recommended for use. Although, if this model is going to be further used for rain loss characterization, the model either needs to be improved to fall within the measurement region, or the differences must be accurately accounted for by taking a lot more data. An increase in the amount of data will ensure that the inaccuracies are truly from the model rather than random outliers in the data set.

The idea of using a rain simulator is amazing in that rain losses can be measured at the designer's desire. This could prove to be extremely advantageous since massive amounts of data can be collected independent of local weather. The use of this specific rain simulator was not particularly ideal due to the vegetation, but it served in proving that the developed model produces results relatively close to the measured results. A lesson learned a little too late was to completely characterize the equipment prior to use. This was a major flaw in the efforts of this research. Specifically, only the antenna gains provided by the manufacturer specification sheets were used in the predictive models.

\section{RECOMMENDATIONS}

It is often said that experimental results are only acceptable if they are repeatable. The first step in continuing this work should be to repeat the rain simulations and anechoic chamber experiments as many times as possible to obtain several data sets for analysis. The collected data will serve as a reference for the designer to continually characterize the model.

Ideally, a unique rain simulator would be created specifically for the purpose of this mission to eliminate any unnecessary losses or unknown variables. A rain simulator could easily be built with raised PVC pipe, rain quality sprinkler heads, a water source, and a generator to pump the water. This would be very beneficial to this cause since measurements could be taken as need or desired. Another advantage in doing this is that experiments could occur during windy conditions to investigate the impact of scintillation and rain together. The challenge here would be to mitigate the reflections. Either special water-proof absorbing material would need to be used, or the antennas would have to be high enough to eliminate any reflection issues.

Once a sufficient amount of data is collected and the model is thought to be characterized completely, then this work should be extended to the V and $\mathrm{W}$ frequency bands. Propagation 
characterization at these bands is the ultimate goal of this experiment. If the developed model is characterized well enough, then it should serve as a good baseline.

Though there are plenty of ways a model can be improved, a few convenient ones that were in the making are listed below.

- The addition of a noise input box was intended to be added as a user input to the GUI. The function noisePow.m was created for this purpose, but was unable to be added in the interest of time. This box will allow the user to obtain accurate carrier-to-noise ratio with knowledge of bandwidth and the noise figure or noise temperature.

- Add the ability to save table data. Though the data can be copied out of the GUI, it is convenient for analysis if the data be output to either a .txt or .xlsx file type.

- Add the ITU-R recommendation for depolarization losses.

- $\quad$ Add the ITU-R low angle fading model. 


\section{REFERENCES}

1. Allnutt, J. E., Satellite-to-Ground Radiowave Propagation, 2nd Edition. London, United Kingdom, The Institution of Engineering and Technology, 2011.

2. Recommendation ITU-R P.838-3, "Specific Attenuation Model for Rain for Use in Prediction Methods," Study Group 3 - Radiowave Propagation, 2005.

3. Recommendation ITU-R P.618-10, "Propagation Data and Prediction Methods Required for the Design of Earth-Space Telecommunication Systems," Study Group 3 Radiowave Propagation, 2009.

4. Stallo, C., Rossi, T., Mukherjee, S., Cianca, E., and Ruggieri, M., "Analysis and Design of a Point-to-Point Radio-Link at W-band for Future Satellite Telecommunication Experiments," IEEE, Issue 6, Jan 2011.

5. Acosta, R., and Banks, J., Advanced Communications Technology Satellite (ACTS), National Aeronautics and Space Administration, Nov 2009.

6. Balanis, C., Advanced Engineering Electromagnetics, Wiley, New York, 2012.

7. Recommendation ITU-R P.676-9, “Attenuation by Atmospheric Gases,” Study Group 3 Radiowave Propagation, 2012.

8. Recommendation ITU-R P.836-4, "Water Vapor: Surface Density and Total Columnar Content," Study Group 3 - Radiowave Propagation, 2009.

9. Recommendation ITU-R P. 837-6, "Characteristics of Precipitation for Propagation Modeling," Study Group 3 - Radiowave Propagation, 2012.

10. Recommendation ITU-R P.839-3, "Rain Height Model for Prediction Methods," Study Group 3 - Radiowave Propagation, 2001.

11. Recommendation ITU-R P.840-5, “Attenuation Due to Clouds and Fog," Study Group 3 Radiowave Propagation, 2012.

12. Islam, M., Elabdin, Z., Elshaikh, O., Khalifa, O., Zahirul, A., Khan, S., and Naji, A., "Prediction of Signal Attenuation Due to Dust Storms Using MIE Scattering," Engineering Journal, Vol. 11, No. 1, 2010.

13. Ishimaru, A., Wave Propagation and Scattering in Random Media, Wiley-IEEE Press, New York, 1999, ISBN-13: 978-0780347175.

14. Goldhirsh, J., "Attenuation and Backscatter from a Derived Two-Dimensional Duststorm Model," IEEE Trans. Antennas Propagation, V. 49, No. 12, pp. 1703-1711, 2001. 
15. Garcia-del-Pino, P., Riera, J., Benarroch, A., "Tropospheric Scintillation With Concurrent Rain Attenuation at $50 \mathrm{GHz}$ in Madrid," IEEE Antennas and Propagation, Vol. 60, No. 3, Mar 2011.

16. Green, H. E., "Propagation Impairment on Ka-band SATCOM Links in Tropical and Equatorial Regions," IEEE Antennas Propagation Magazine, Vol. 48, No. 2, pp. 31-45, 2004.

17. Goldsmith, A., Wireless Communications, Cambridge University Press, 2005. 
LIST OF SYMBOLS, ABBREVIATIONS AND ACRONYMS

\begin{tabular}{|l|l|}
\hline ACTS & Advanced Communication Technology Satellite \\
\hline CW & Continuous Wave \\
\hline EHF & Extremely High Frequency \\
\hline EIRP & Effective Isotropic Radiated Power \\
\hline FCC & Federal Communications Commission \\
\hline GHz & Giga Hertz \\
\hline GUI & Graphical User Interface \\
\hline ITU & International Telecommunications Union \\
\hline LOS & Line of Sight \\
\hline RF & Radio Frequency \\
\hline RX & Receiver Power Level \\
\hline SMA & Sub-Miniature version A \\
\hline TX & Transmit Power Level \\
\hline UNM & University of New Mexico \\
\hline
\end{tabular}




\section{DISTRIBUTION LIST}

DTIC/OCP

8725 John J. Kingman Rd, Suite 0944

Ft Belvoir, VA 22060-6218

$1 \mathrm{cy}$

AFRL/RVIL

Kirtland AFB, NM 87117-5776

2 cys

Official Record Copy

AFRL/RVSV/Steven A. Lane 1 cy

Approved for Public Release; Distribution is Unlimited. 
(This page intentionally left blank)

Approved for Public Release; Distribution is Unlimited. 